\title{
Potent Cytotoxic Arylnaphthalene Lignan Lactones from
}

\section{Phyllanthus poilanei}

Yulin Ren, ${ }^{\dagger}$ Daniel D. Lantvit, ${ }^{\ddagger}$ Youcai Deng, ${ }^{\S}$ Ragu Kanagasabai, ${ }^{\perp}$ Judith C. Gallucci, $\|$ Tran

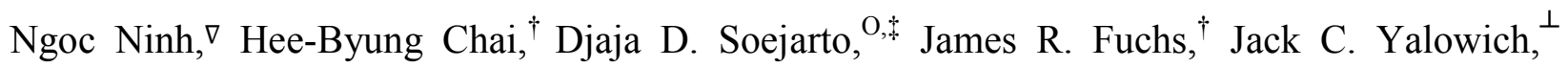
Jianhua Yu, ${ }^{\S, \#}$ Steven M. Swanson, ${ }^{\ddagger}$ and A. Douglas Kinghorn ${ }^{*}, \dagger$

${ }^{\dagger}$ Division of Medicinal Chemistry and Pharmacognosy, College of Pharmacy, The Ohio State University, Columbus, $\mathrm{OH} 43210$, United States

*Department of Medicinal Chemistry and Pharmacognosy, College of Pharmacy, University of Illinois at Chicago, Chicago, IL 60612, United States

${ }^{\S}$ Division of Hematology, Department of Internal Medicine, College of Medicine, The Ohio State University, Columbus, OH 43210, United States

${ }^{\perp}$ Division of Pharmacology, College of Pharmacy, The Ohio State University, Columbus, Ohio 43210, United States

II Department of Chemistry and Biochemistry, The Ohio State University, Columbus, OH 43210, United States

${ }^{\nabla}$ Institute of Ecology and Biological Resources, Vietnam Academy of Science and Technology, Hoang Quoc Viet, Cau Giay, Hanoi, Vietnam

${ }^{\mathrm{O}}$ Department of Botany, Field Museum of Natural History, Chicago, IL 60605, United States

${ }^{\#}$ Comprehensive Cancer Center, The Ohio State University, Columbus, OH 43210, United States 


\section{Content of Supporting Information}

Figure S1. MS spectrum of phyllanthusmin D (1).

Figure S2. NMR spectrum of phyllanthusmin D (1).

Figure S3. MS spectrum of phyllanthusmin E (2).

Figure S4. NMR spectrum of phyllanthusmin E (2).

Figure S5. MS spectrum of phyllanthusmin B (3).

Figure S6. NMR spectrum of phyllanthusmin B (3).

Figure S7. MS spectrum of phyllanthusmin C (4).

Figure S8. NMR spectrum of phyllanthusmin C (4).

Figure S9. MS spectrum of cleistanthin B (5).

Figure S10. NMR spectrum of cleistanthin B (5).

Figure S11. MS spectrum of phyllanthusmin A (6).

Figure S12. NMR spectrum of phyllanthusmin A (6).

Figure S13. MS spectrum of 7-O-[(2,3,4-tri- $O$-acetyl)- $\alpha$-L-arabinopyranosyl)]diphyllin (7).

Figure S14. NMR spectrum of 7- $O$-[(2,3,4-tri- $O$-acetyl)- $\alpha$-L-arabinopyranosyl)]diphyllin (7).

Figure S15. MS spectrum of diphyllin (8).

Figure S16. NMR spectrum of diphyllin (8).

Figure S17. ECD spectra of phyllanthusmins D (1) and E (2).

Figure S18. COSY and key HMBC correlations of 2-8.

Figure S19. Selected NOESY correlations of 2-5 and 7.

Tables S1-S4. ${ }^{1} \mathrm{H}$ and ${ }^{13} \mathrm{C}$ NMR spectroscopic data of 3-8.

Analytical data of known compounds (3-8) isolated from Phyllanthus poilanei. 


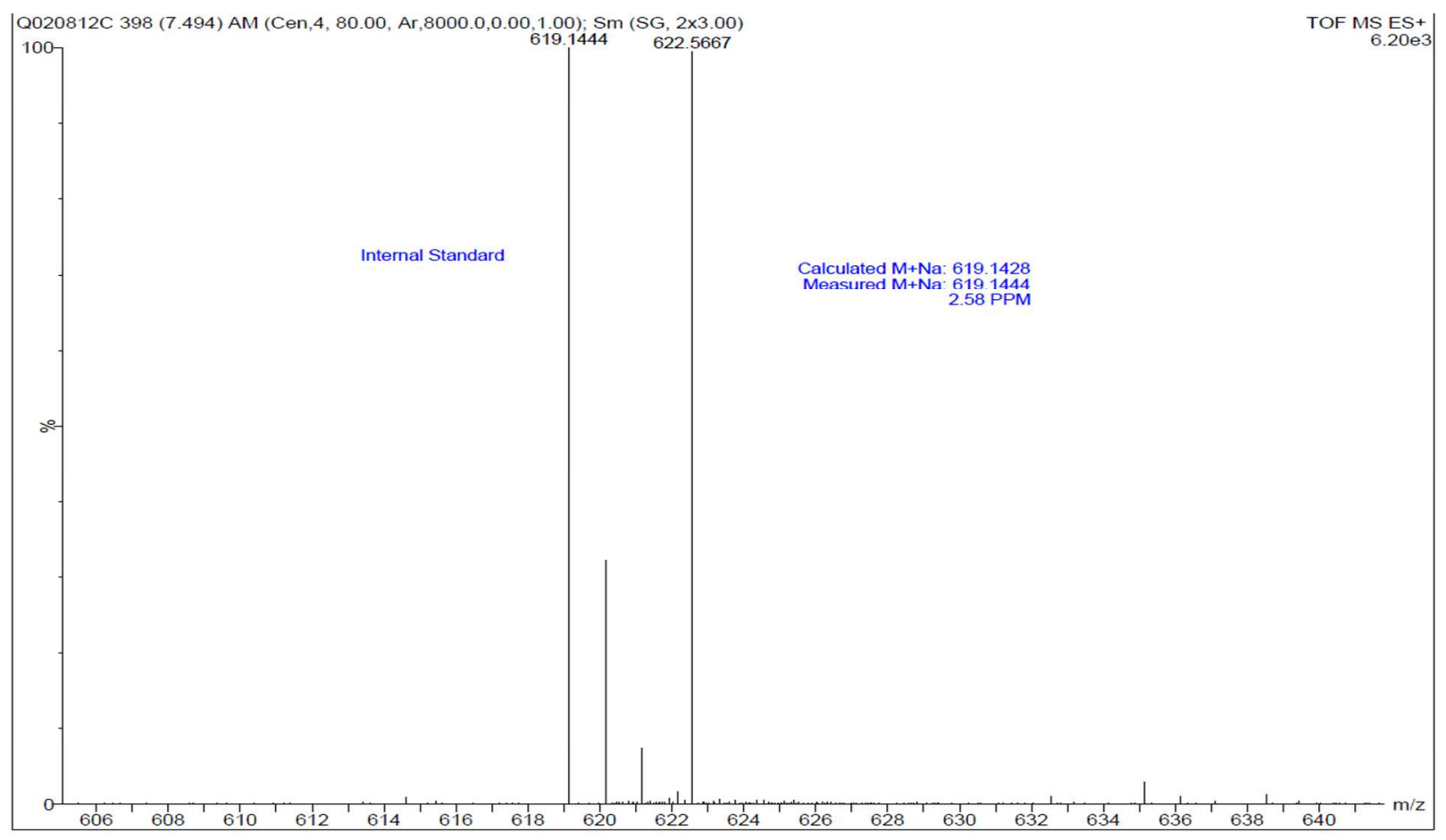

Figure S1. MS spectrum of phyllanthusmin D (1).

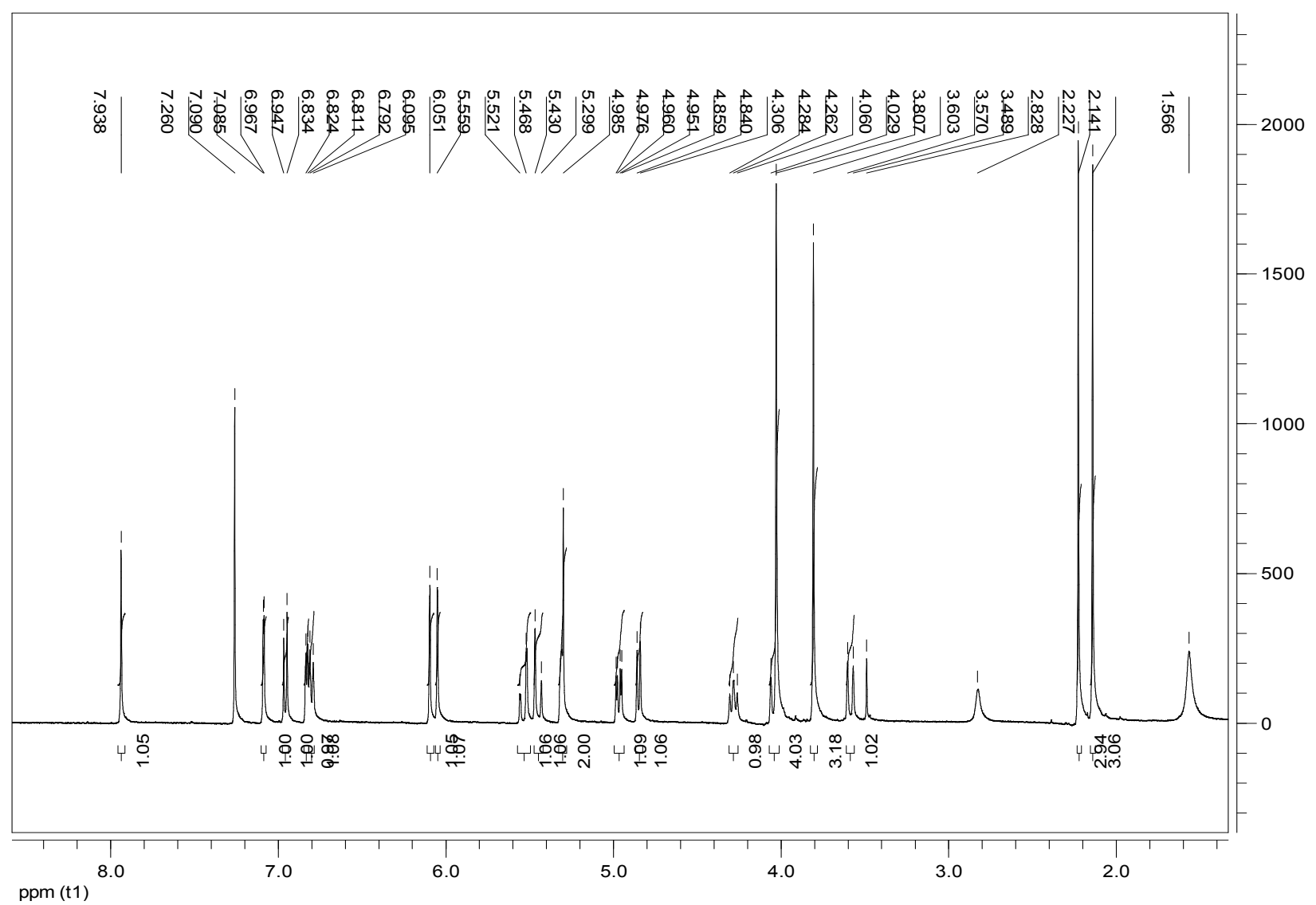




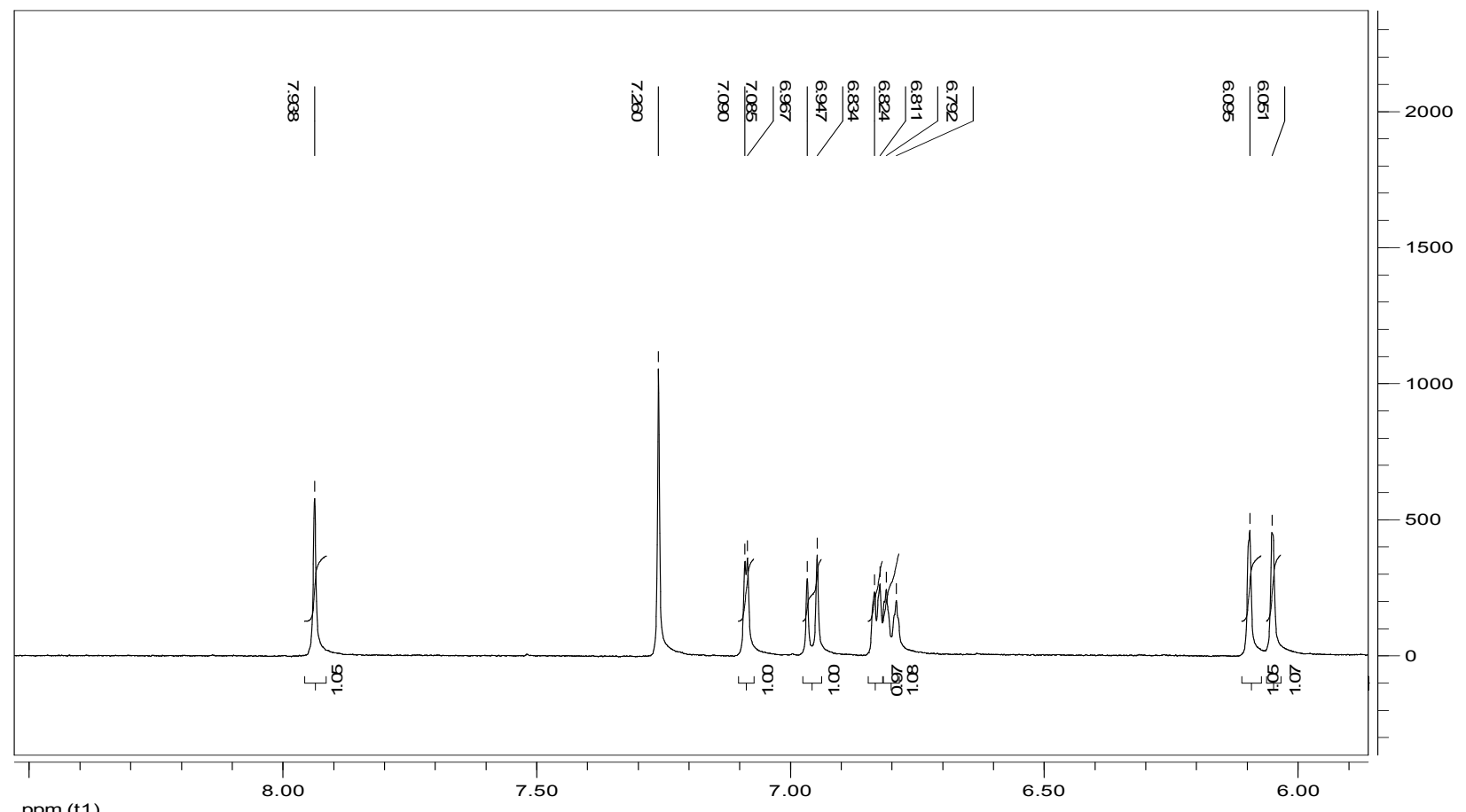

ppm (t1)

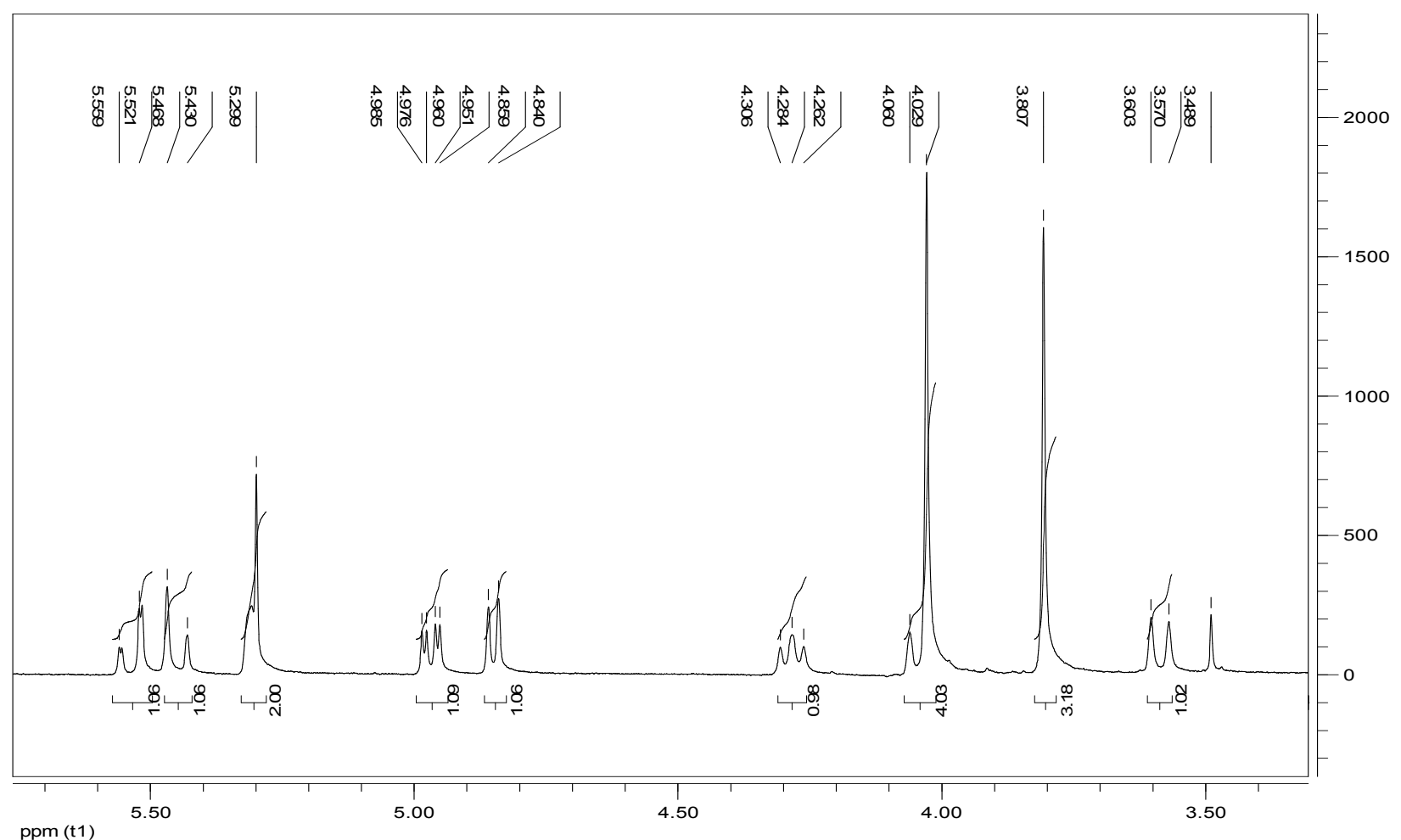

Figure S2a. ${ }^{1}$ H NMR spectrum phyllanthusmin D (1). 


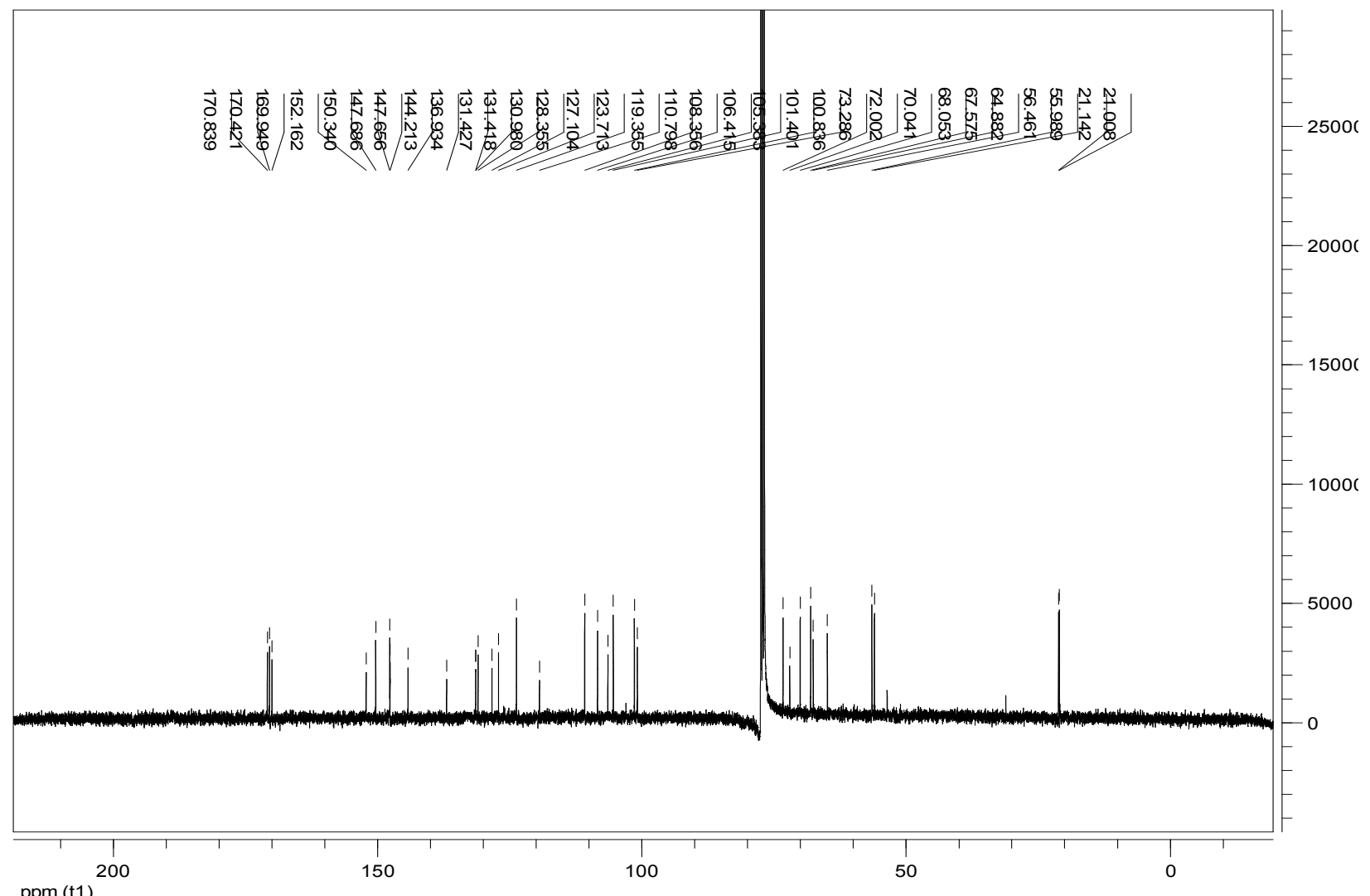
ppm (t1)

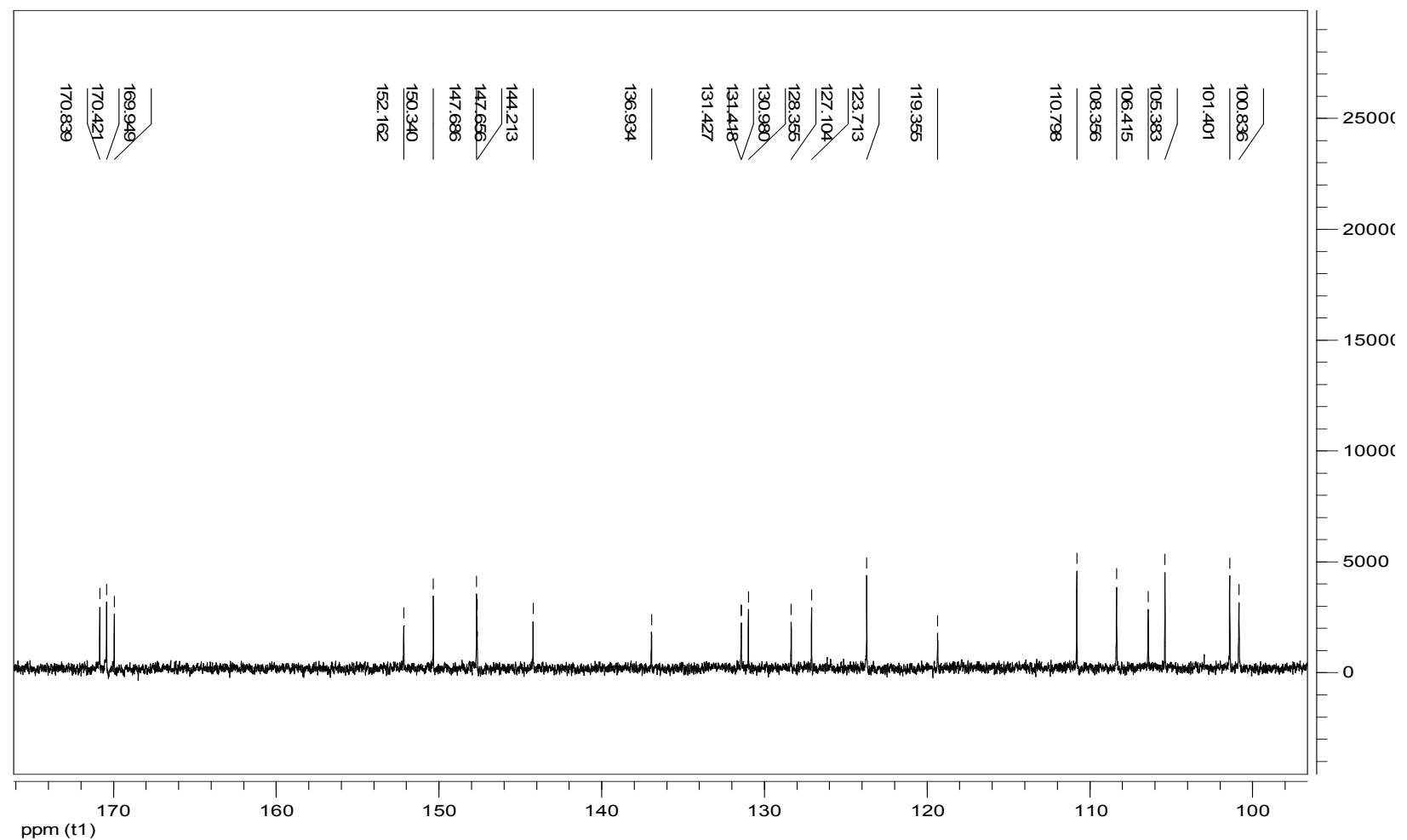




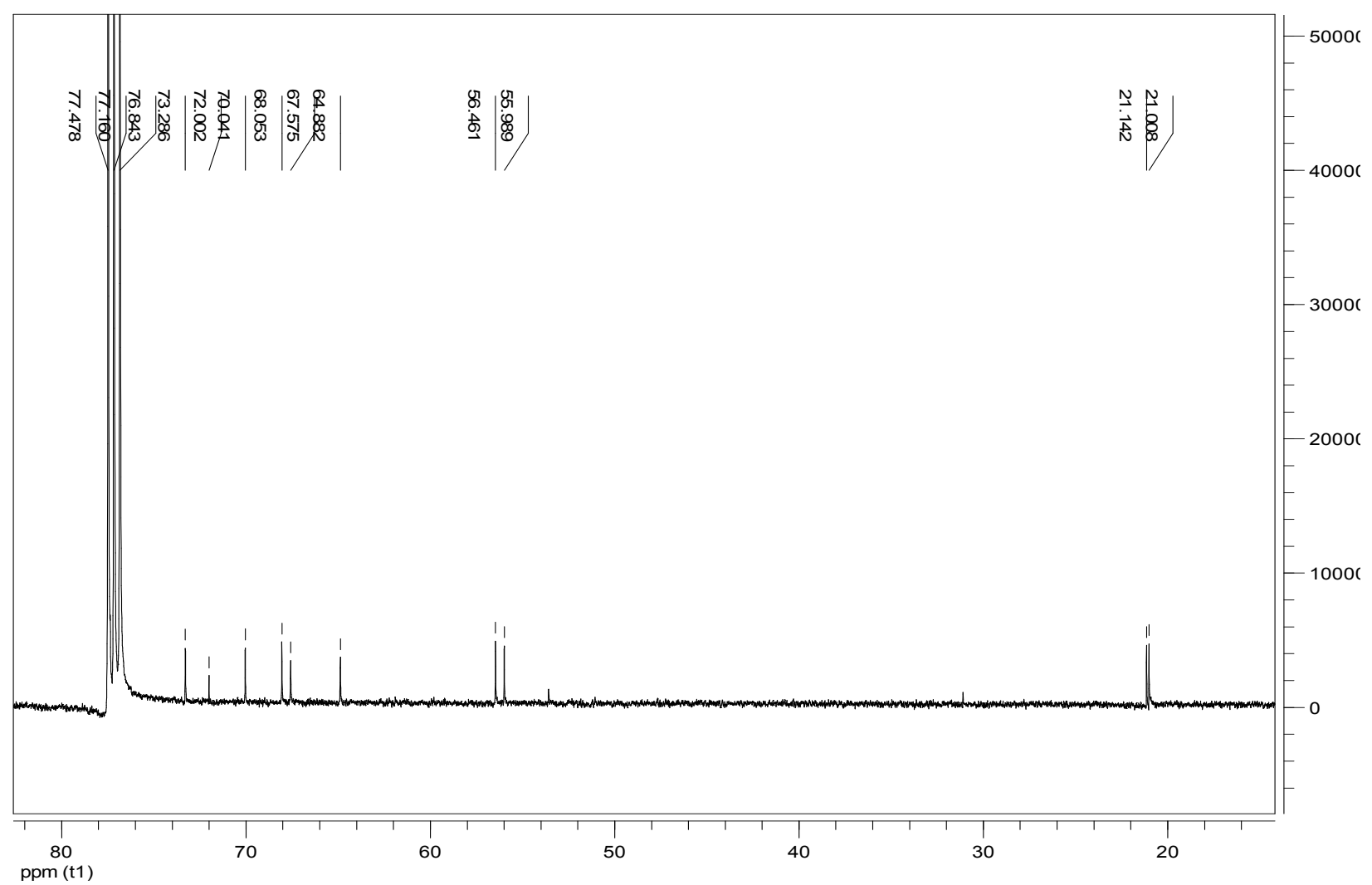

Figure S2b. ${ }^{13}$ C NMR spectrum phyllanthusmin D (1).

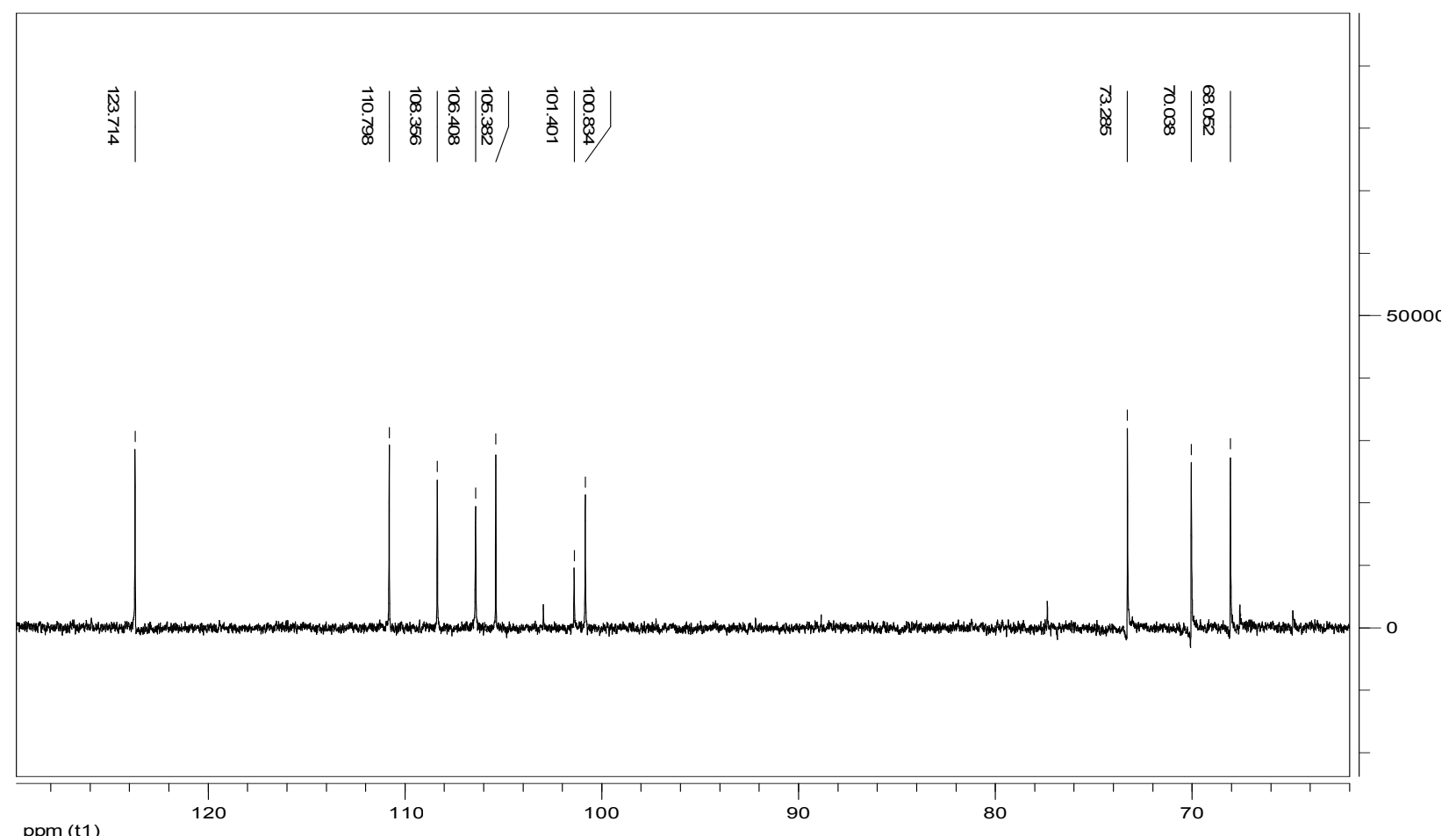

Figure S2c. DEPT 90 NMR spectrum phyllanthusmin D (1). 


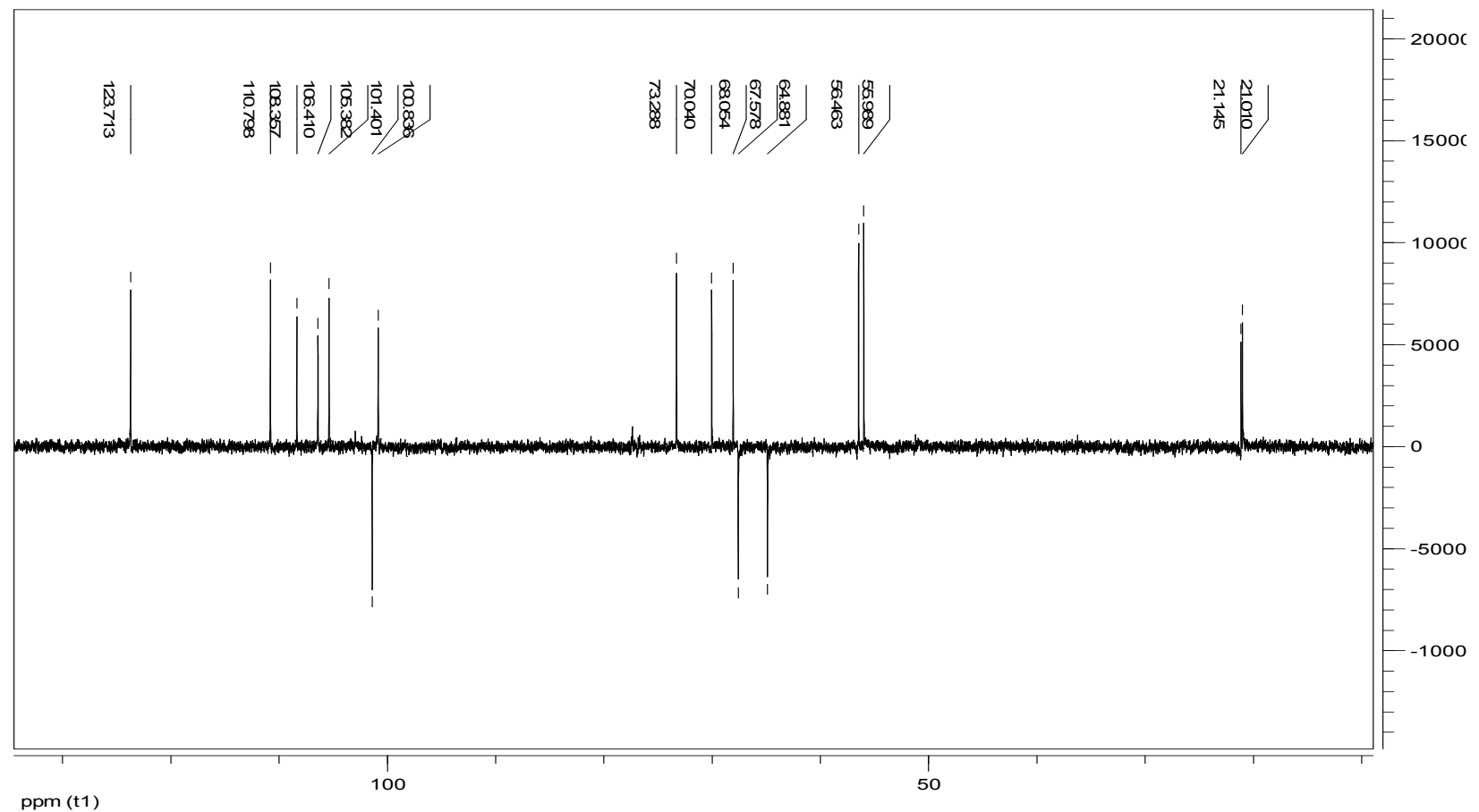

Figure S2d. DEPT 135 NMR spectrum phyllanthusmin D (1).

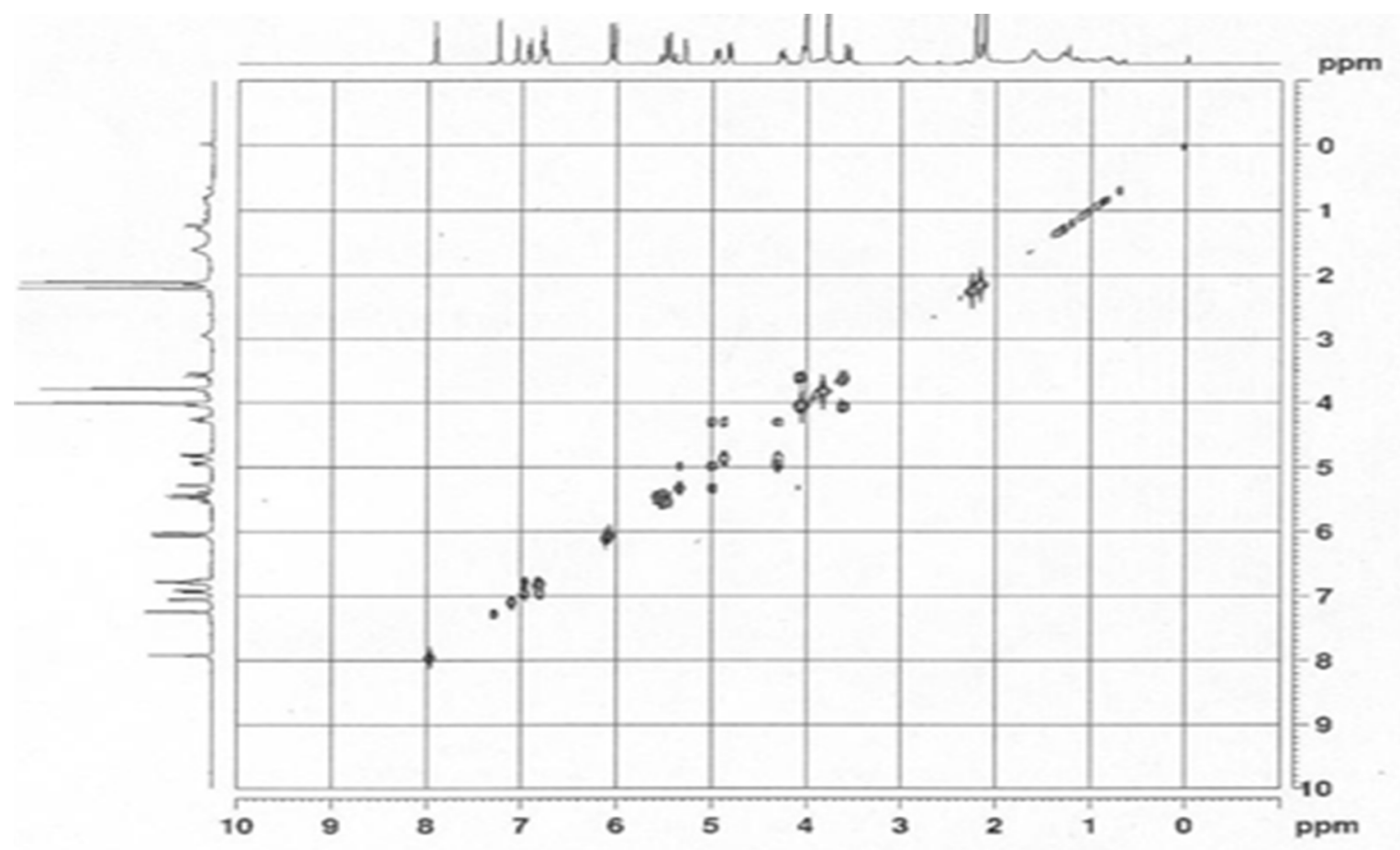



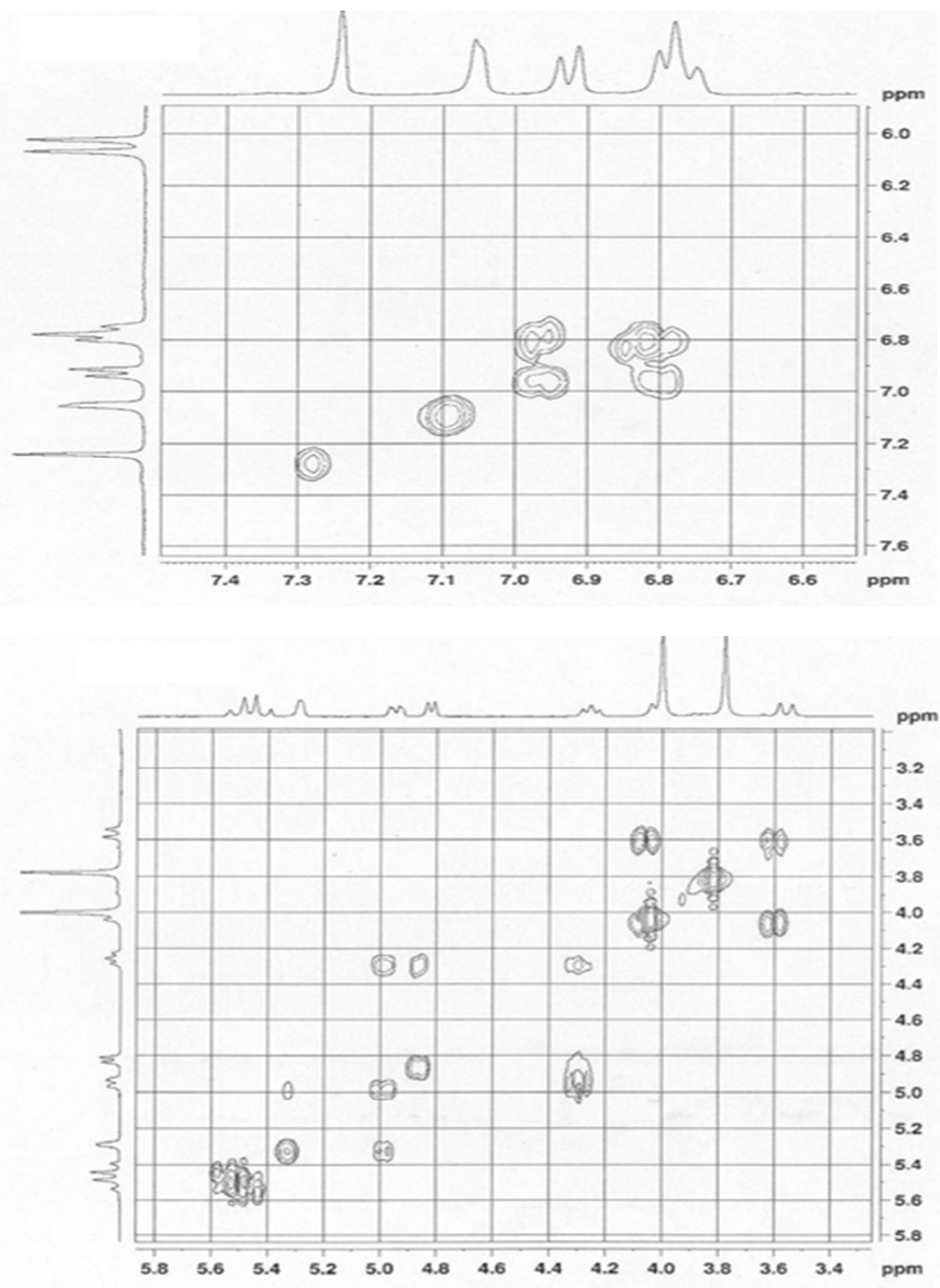

Figure S2e. COSY NMR spectrum phyllanthusmin D (1). 

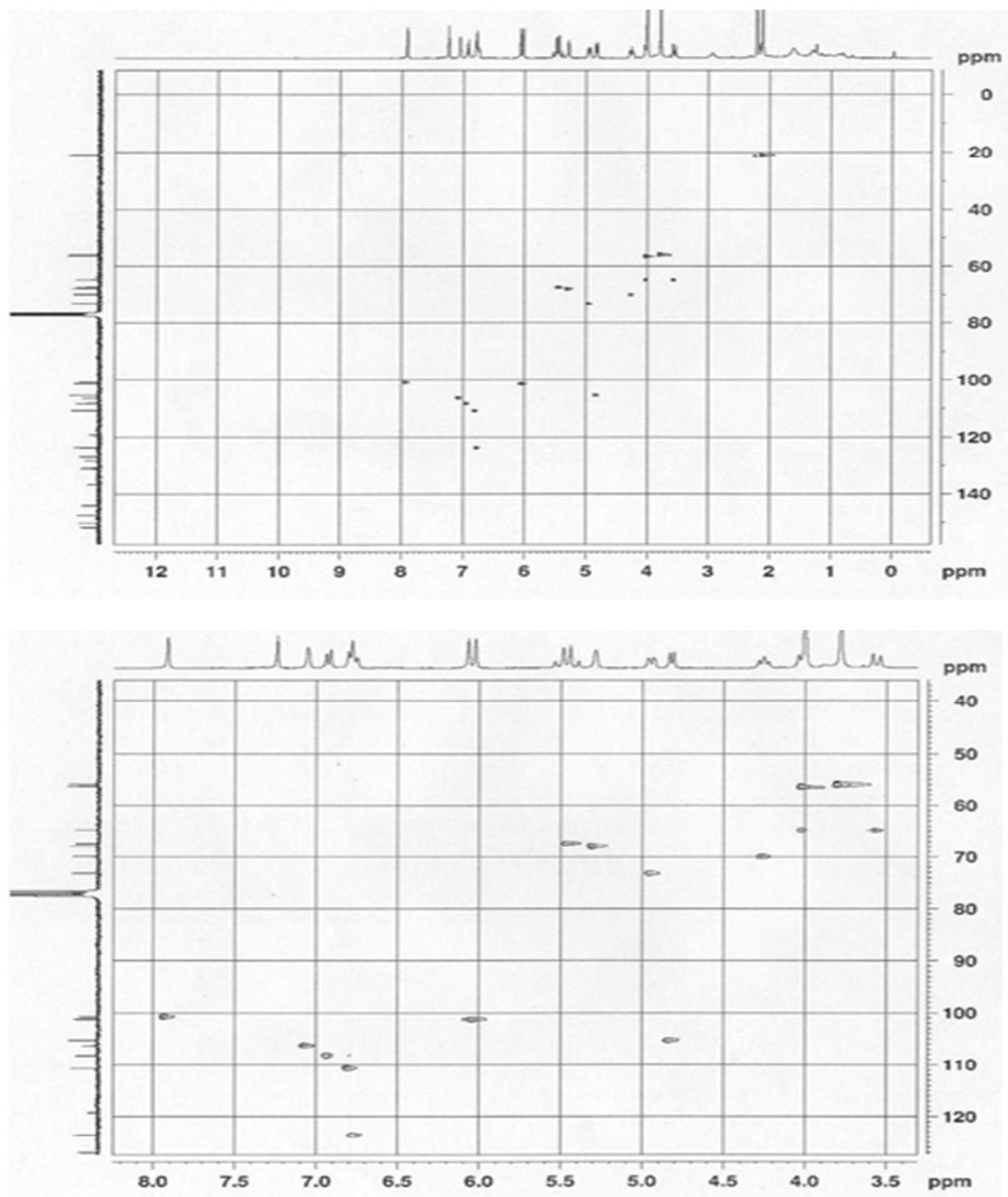

Figure S2f. HSQC 2D NMR spectrum phyllanthusmin D (1). 


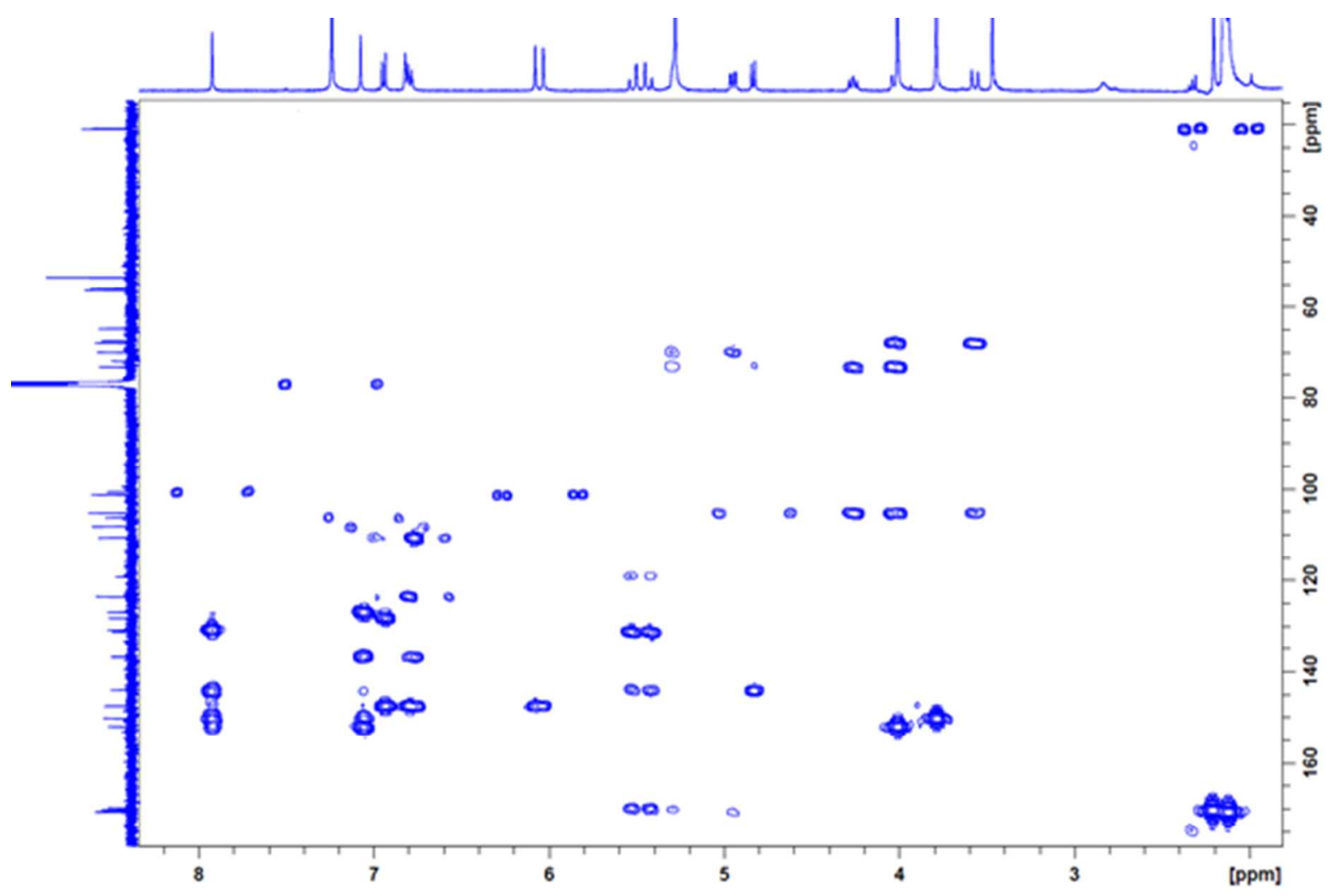

Figure S2g. HMBC 2D NMR spectrum phyllanthusmin D (1).

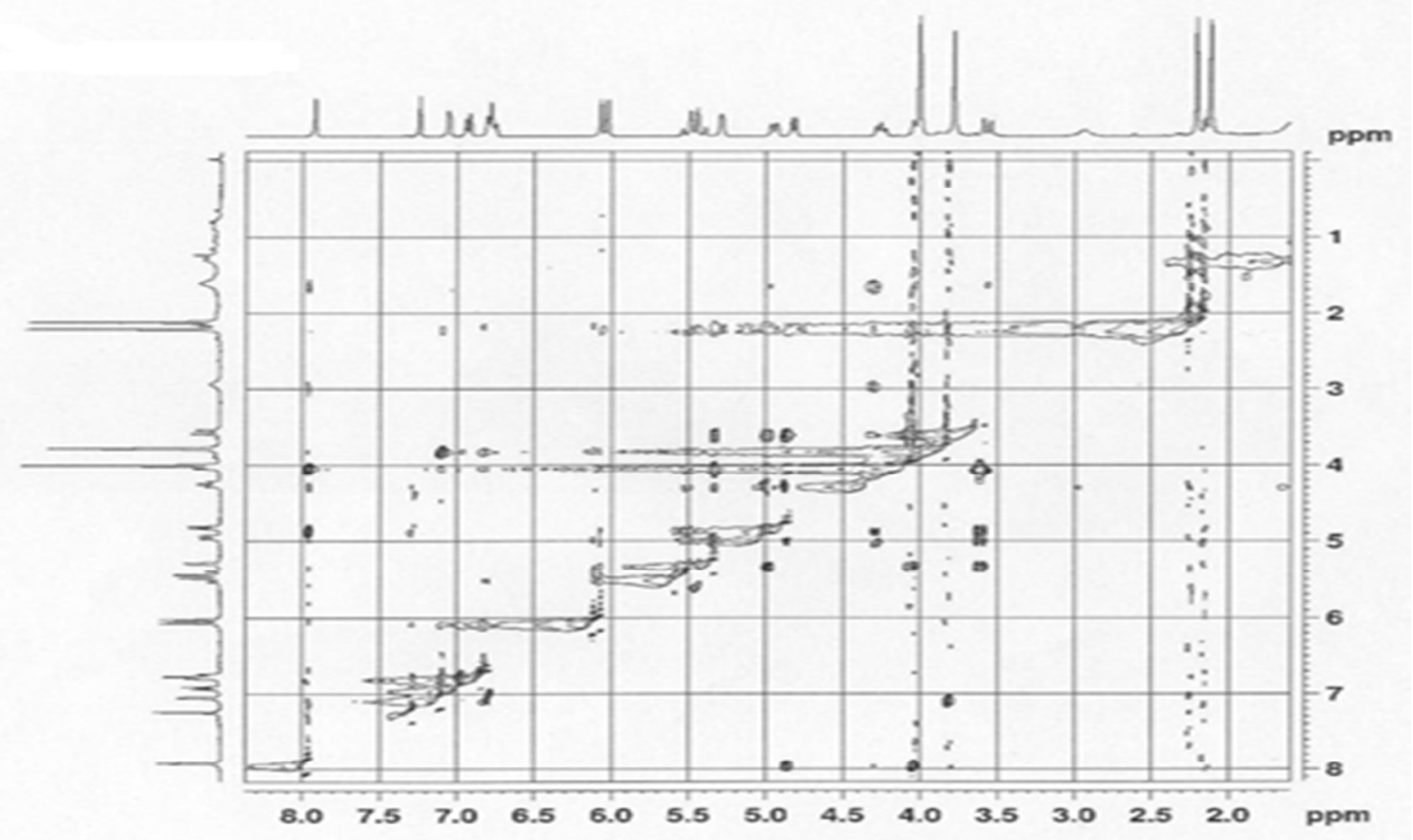

Figure S2h. NOESY 2D NMR spectrum phyllanthusmin D (1). 


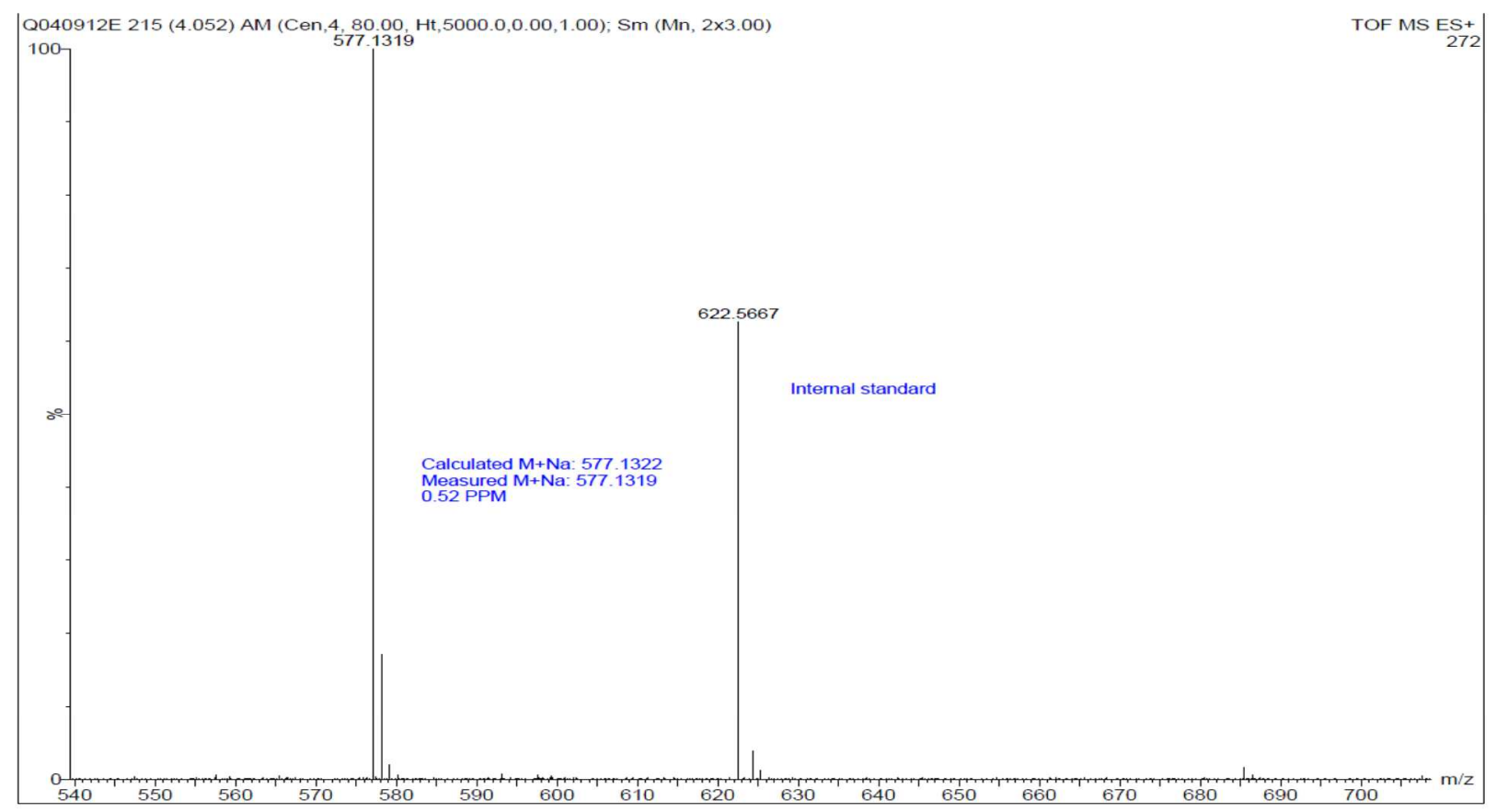

Figure S3. MS spectrum of phyllanthusmin E (2).

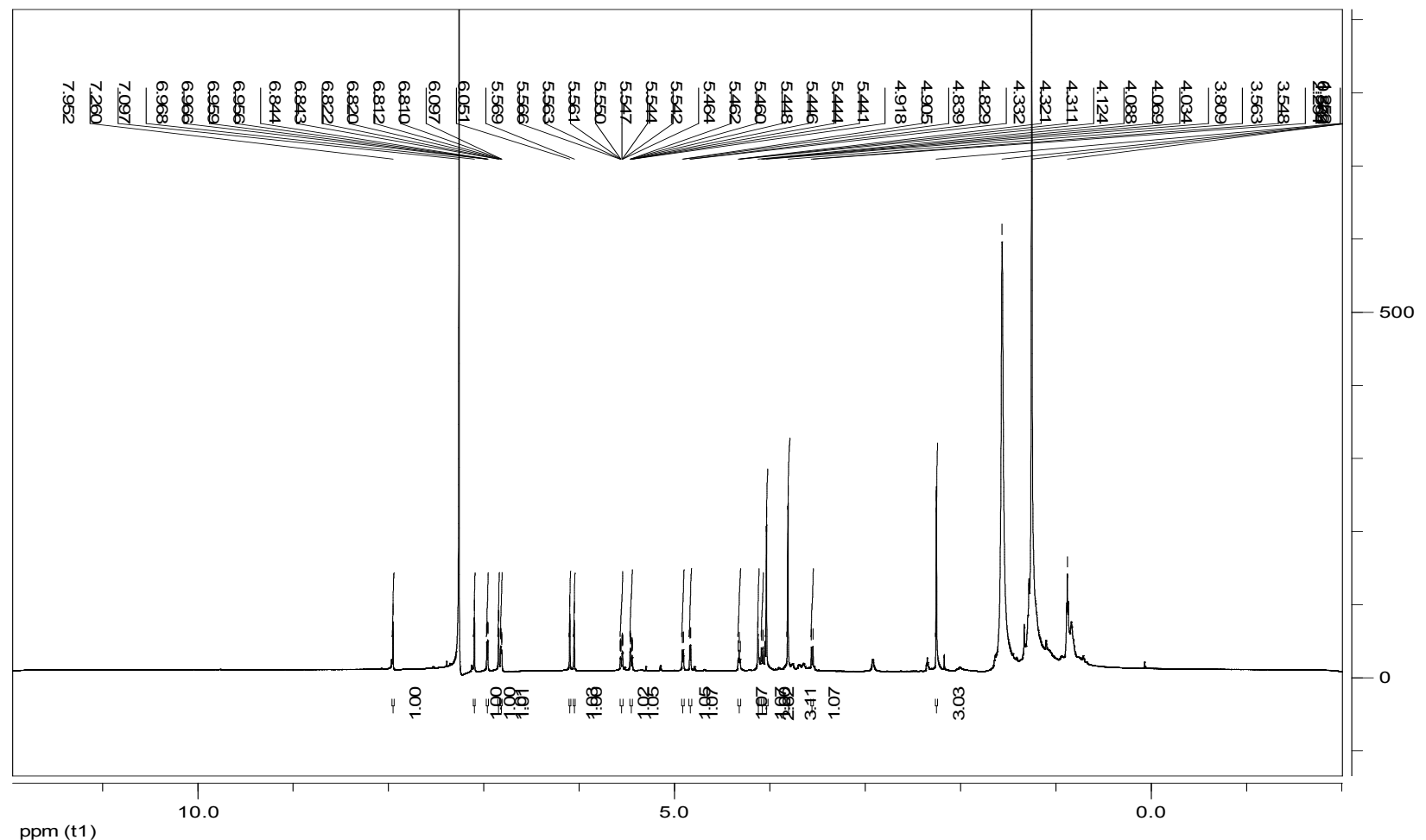



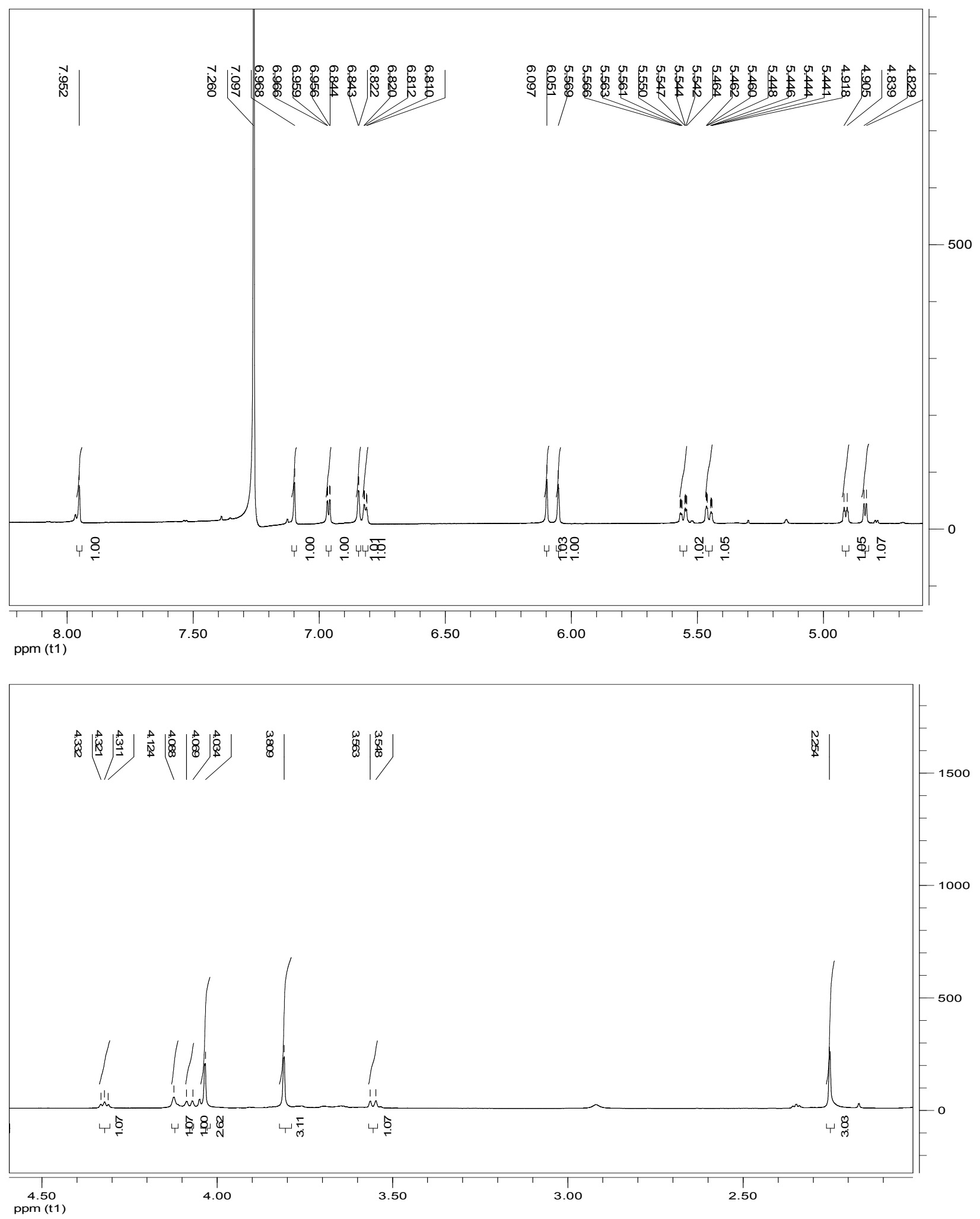

Figure S4a. ${ }^{1} \mathrm{H}$ NMR spectrum of phyllanthusmin E (2). 


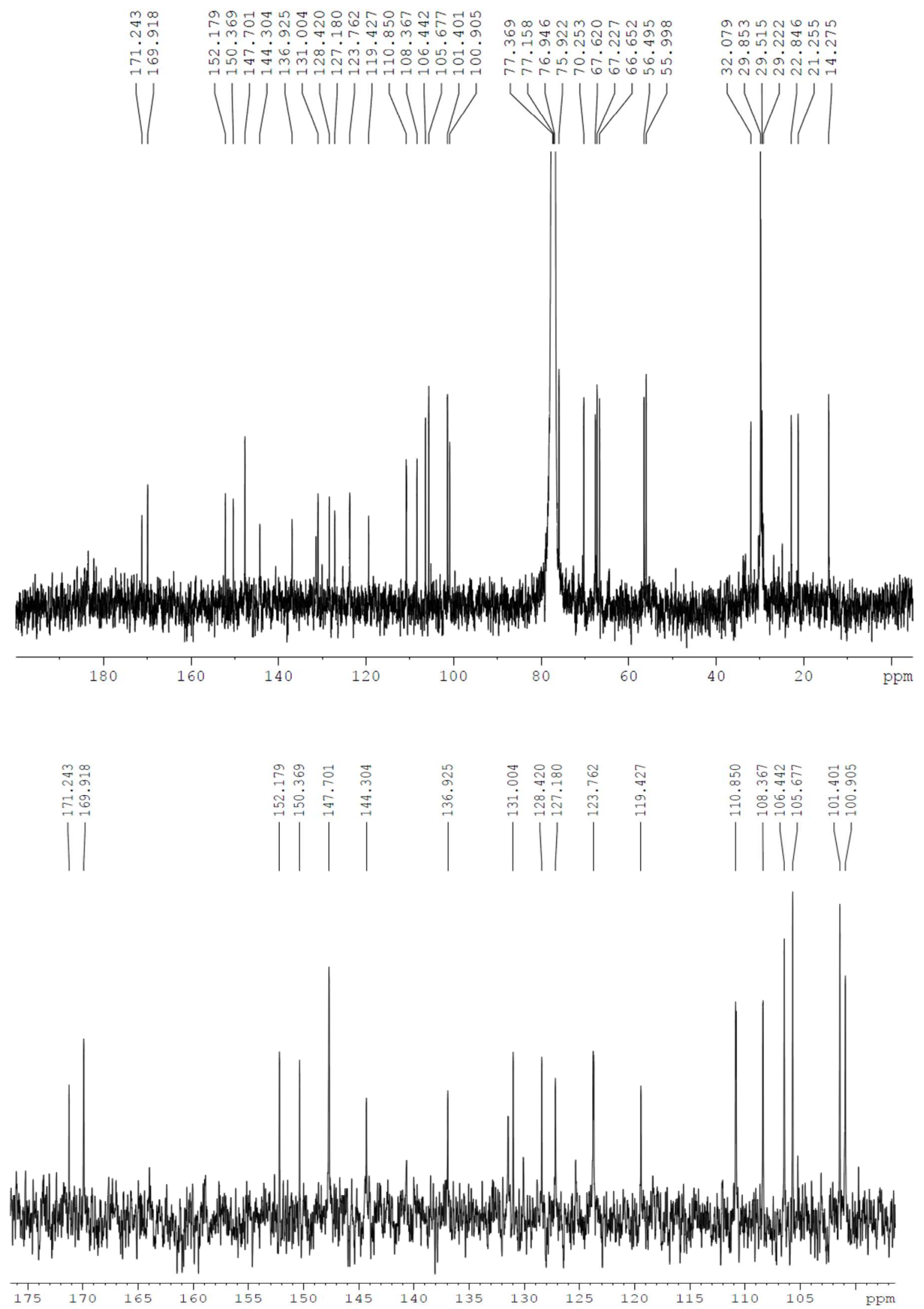




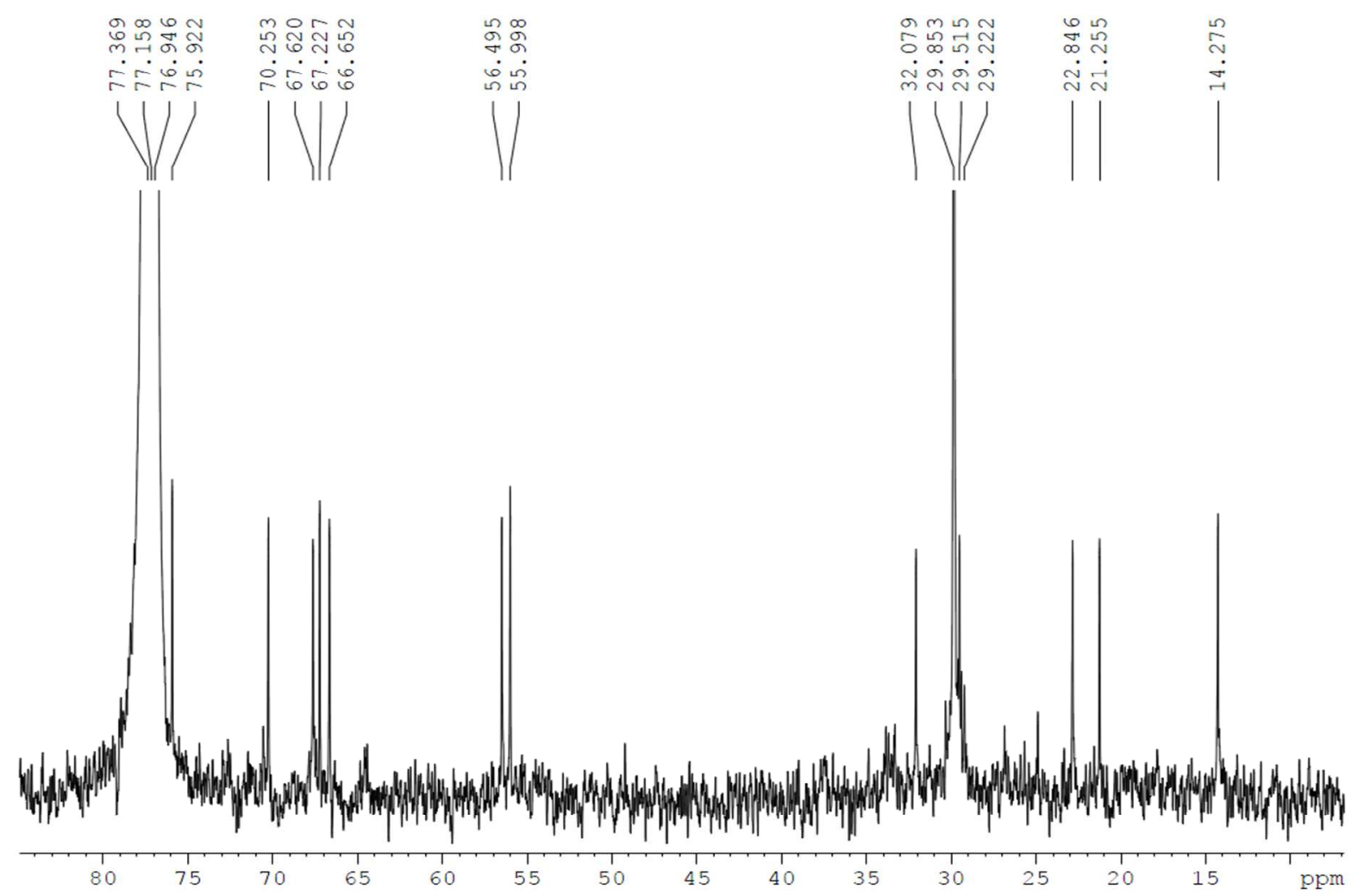

Figure S4b. ${ }^{13} \mathrm{C}$ NMR spectrum of phyllanthusmin E (2). Note: the signals at $\delta_{\mathrm{C}} 14.3,22.8$, and 32.1 are due to $n$-hexane present in the solvent system used. ${ }^{34}$

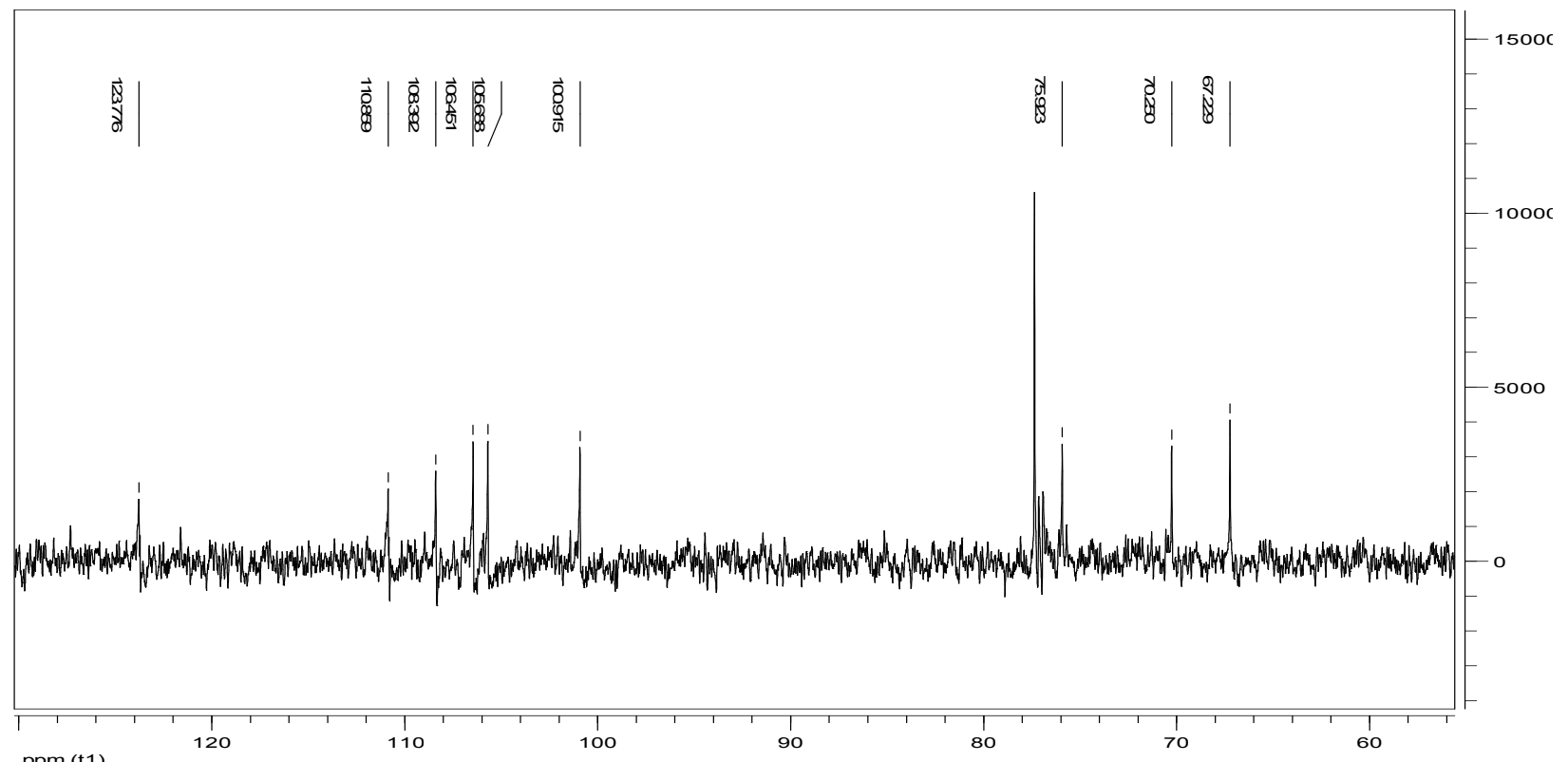

Figure S4c. DEPT 90 NMR spectrum of phyllanthusmin E (2). 


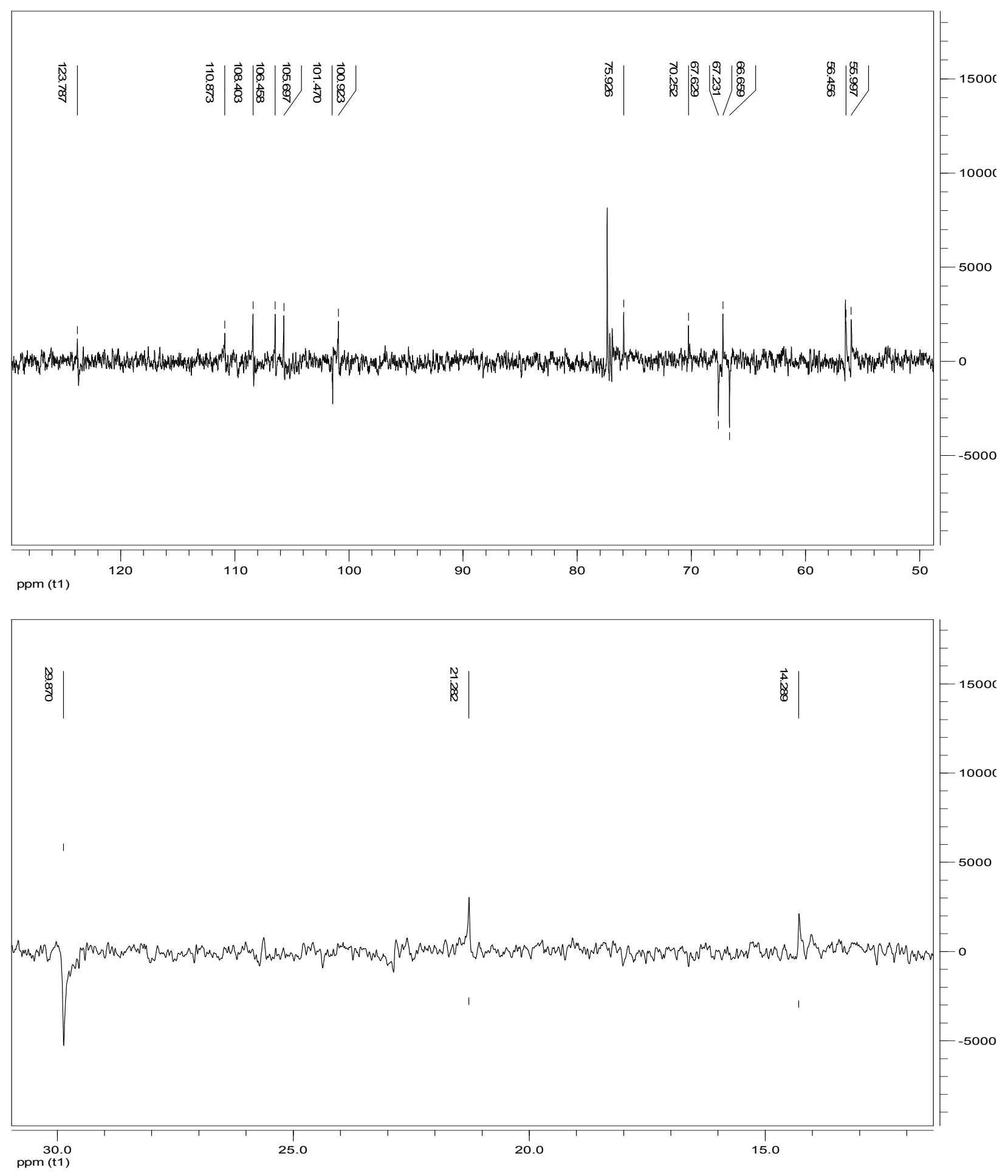

Figure S4d. DEPT 135 NMR spectrum of phyllanthusmin E (2). 


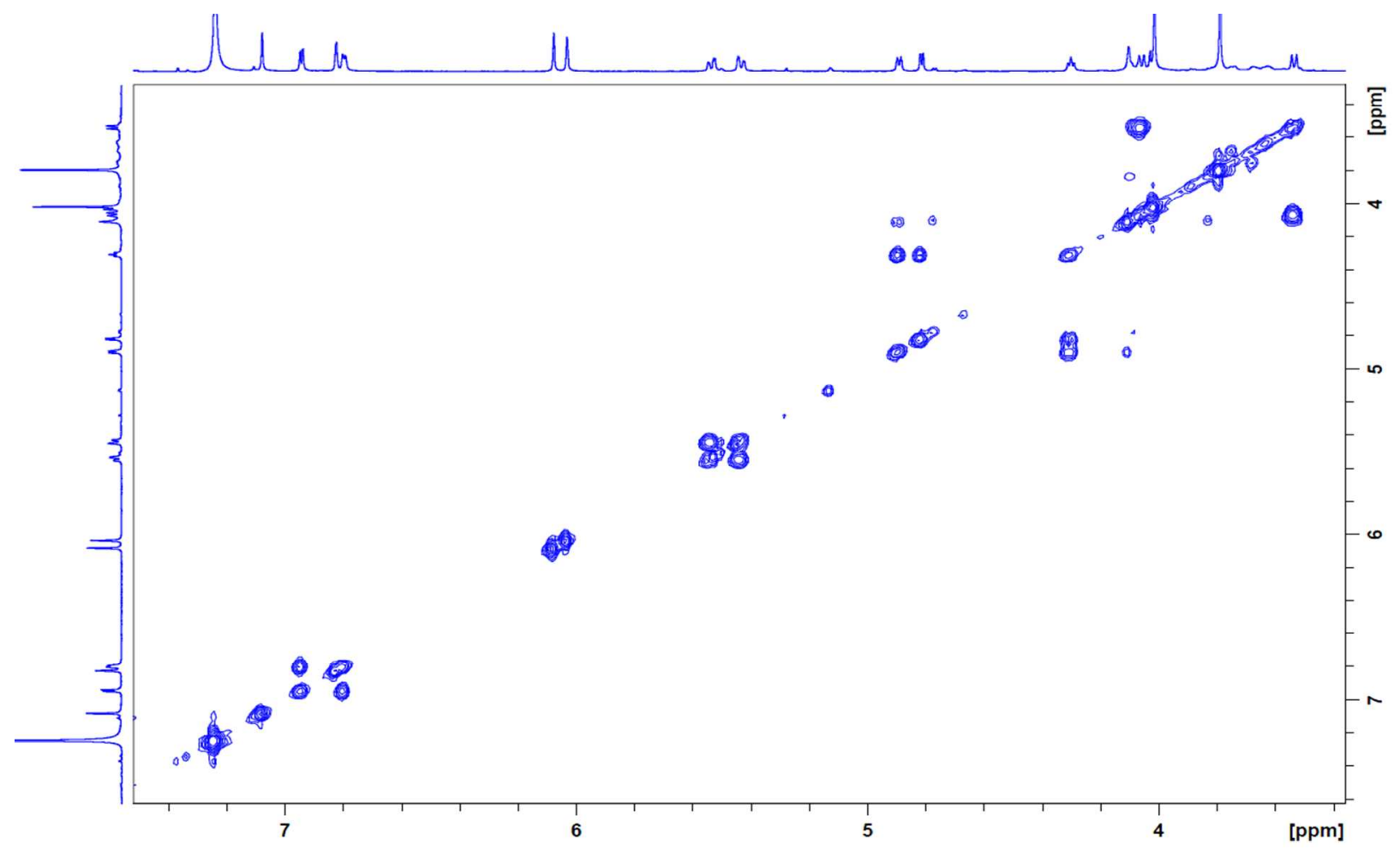

Figure S4e COSY NMR spectrum of phyllanthusmin E (2).

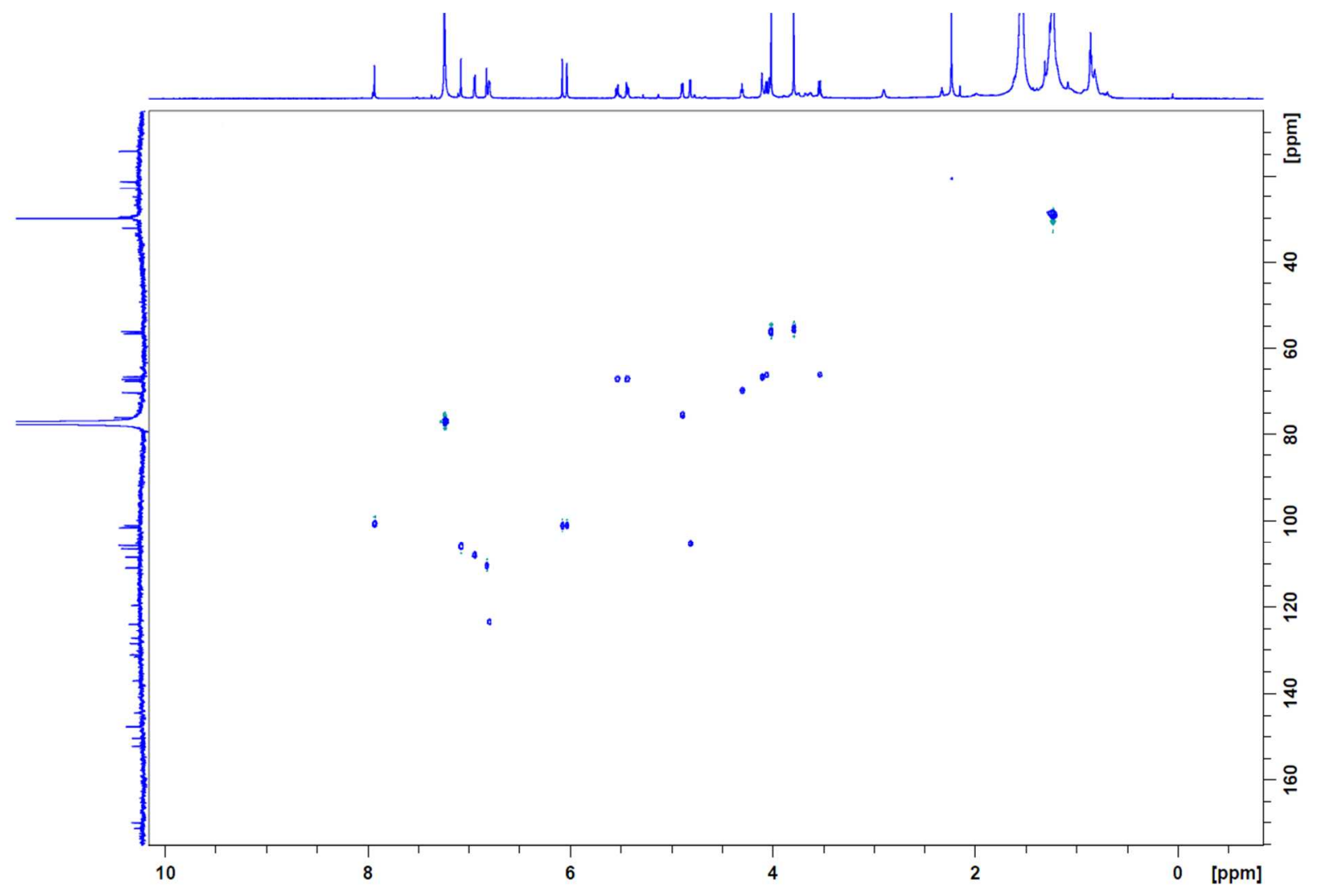




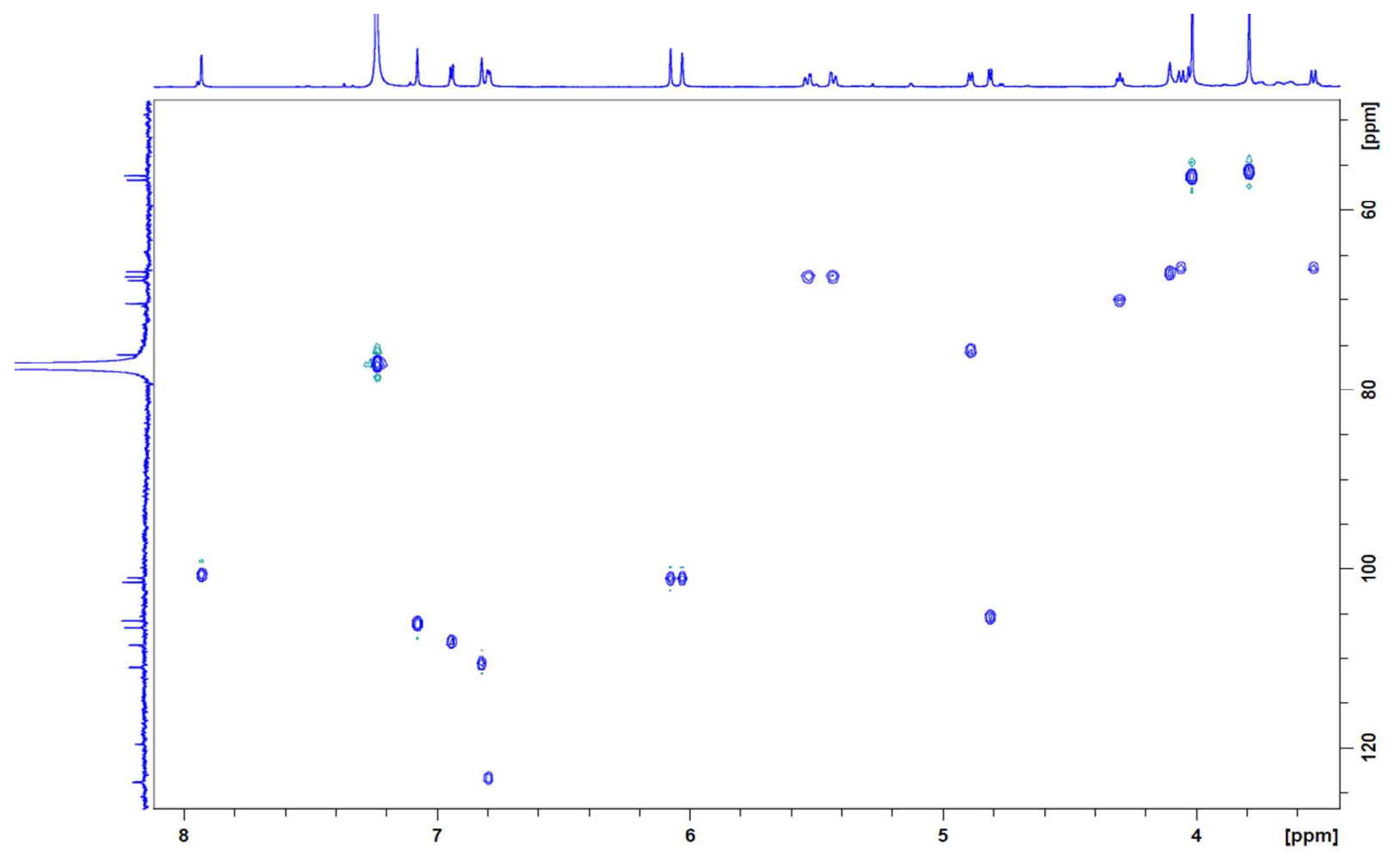

Figure S4f. HSQC 2D NMR spectrum of phyllanthusmin E (2).

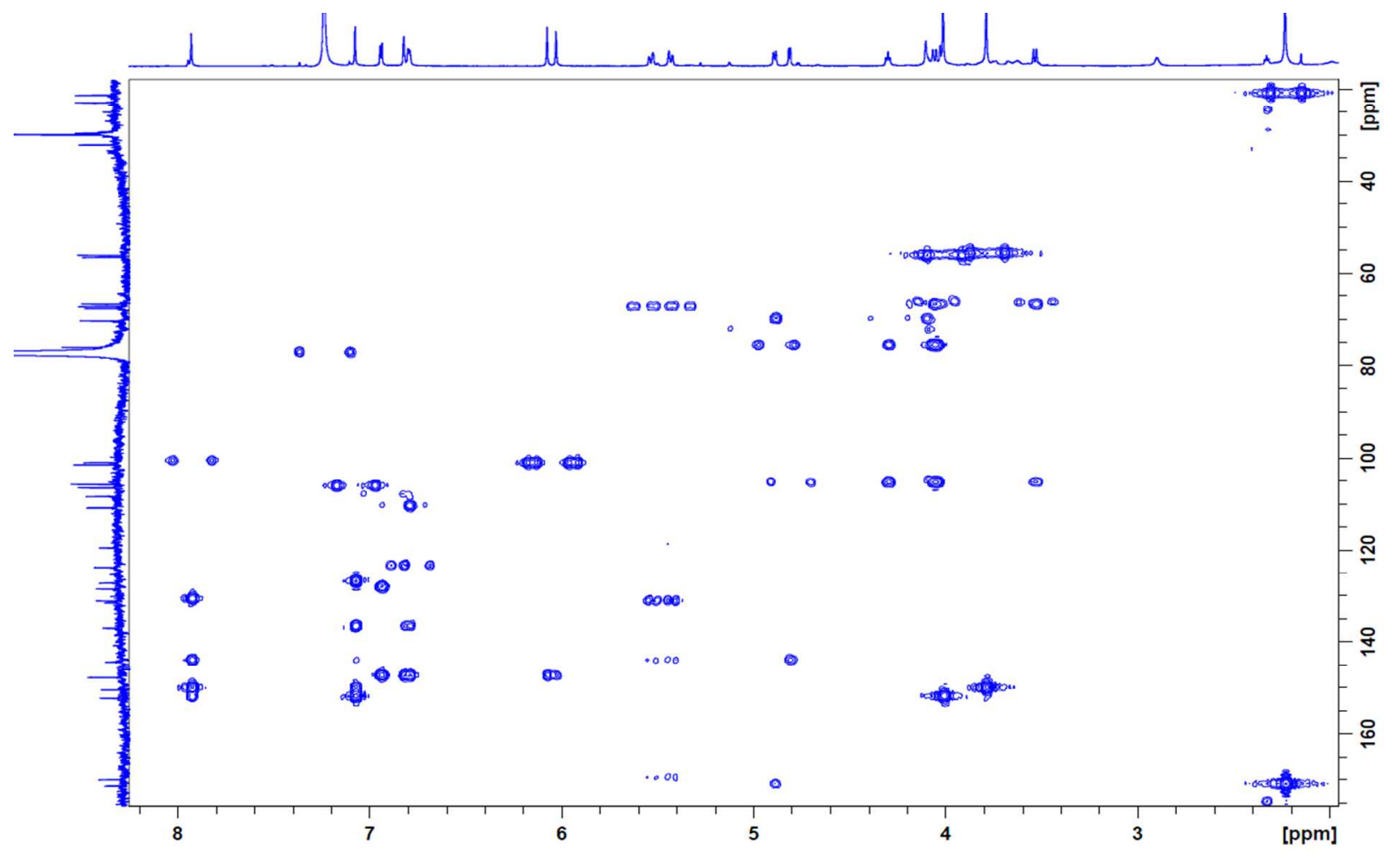

Figure S4g. HMBC 2D NMR spectrum of phyllanthusmin E (2). 


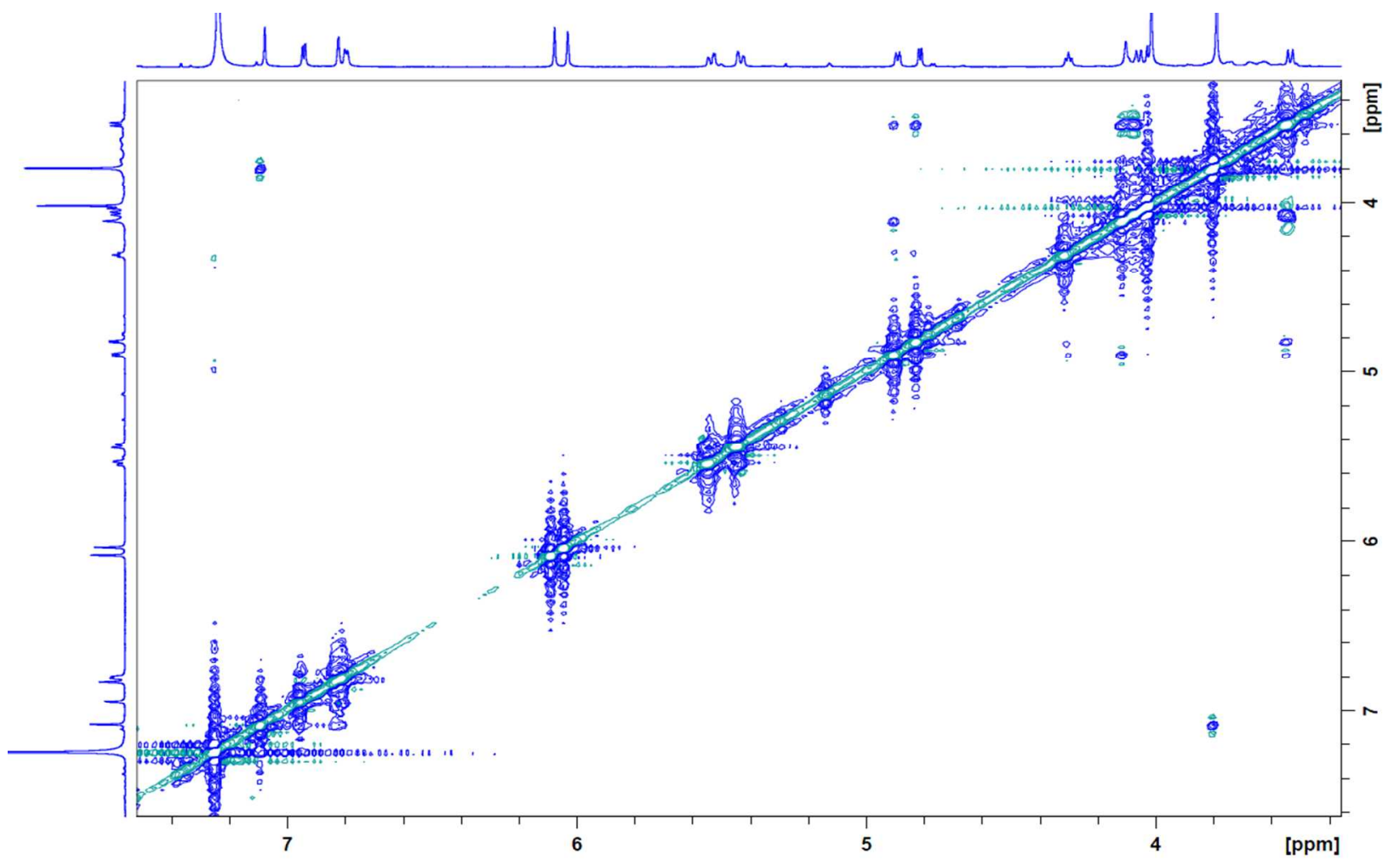

Figure S4h. NOESY 2D NMR spectrum of phyllanthusmin E (2).

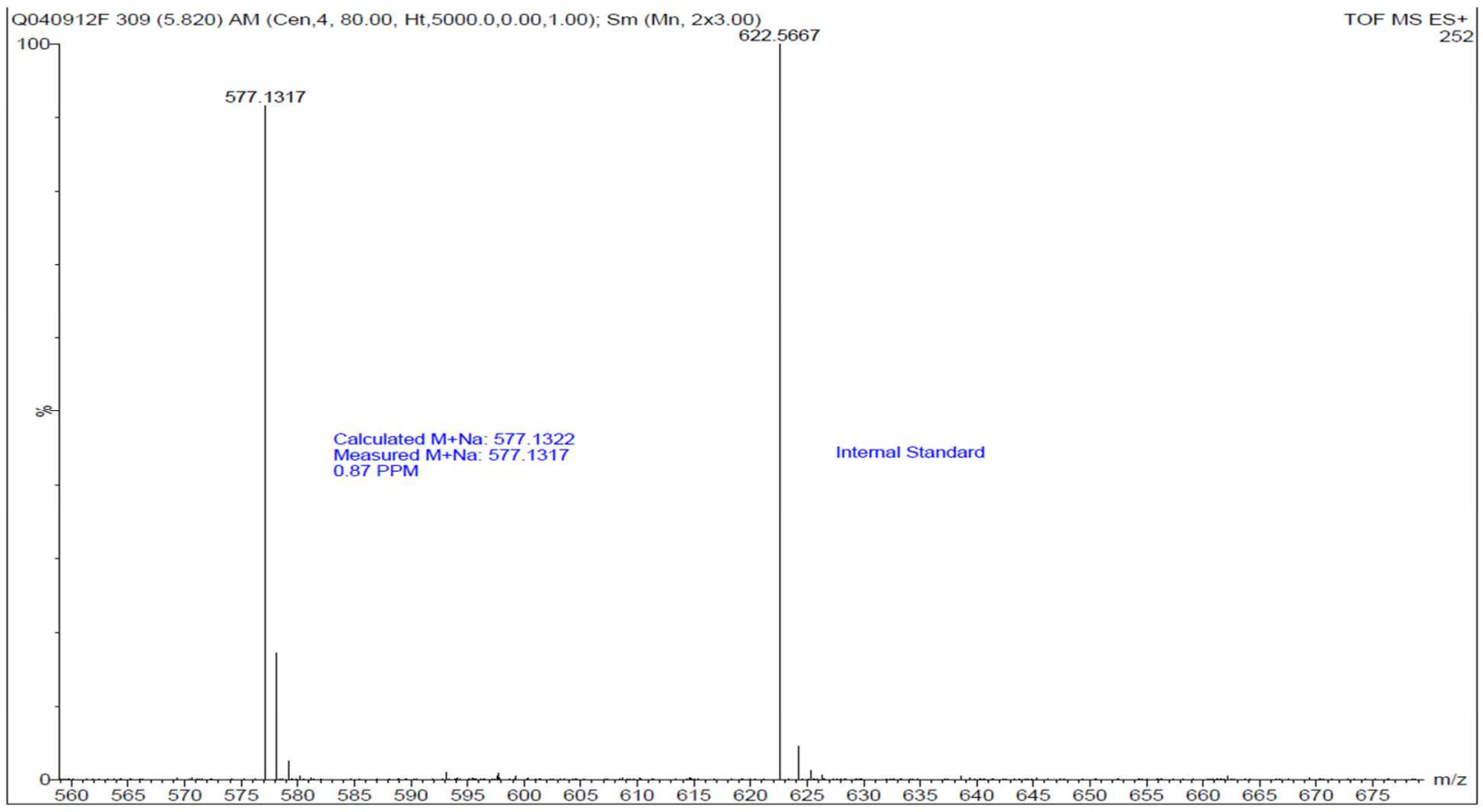

Figure S5. MS spectrum of phyllanthusmin B (3). 

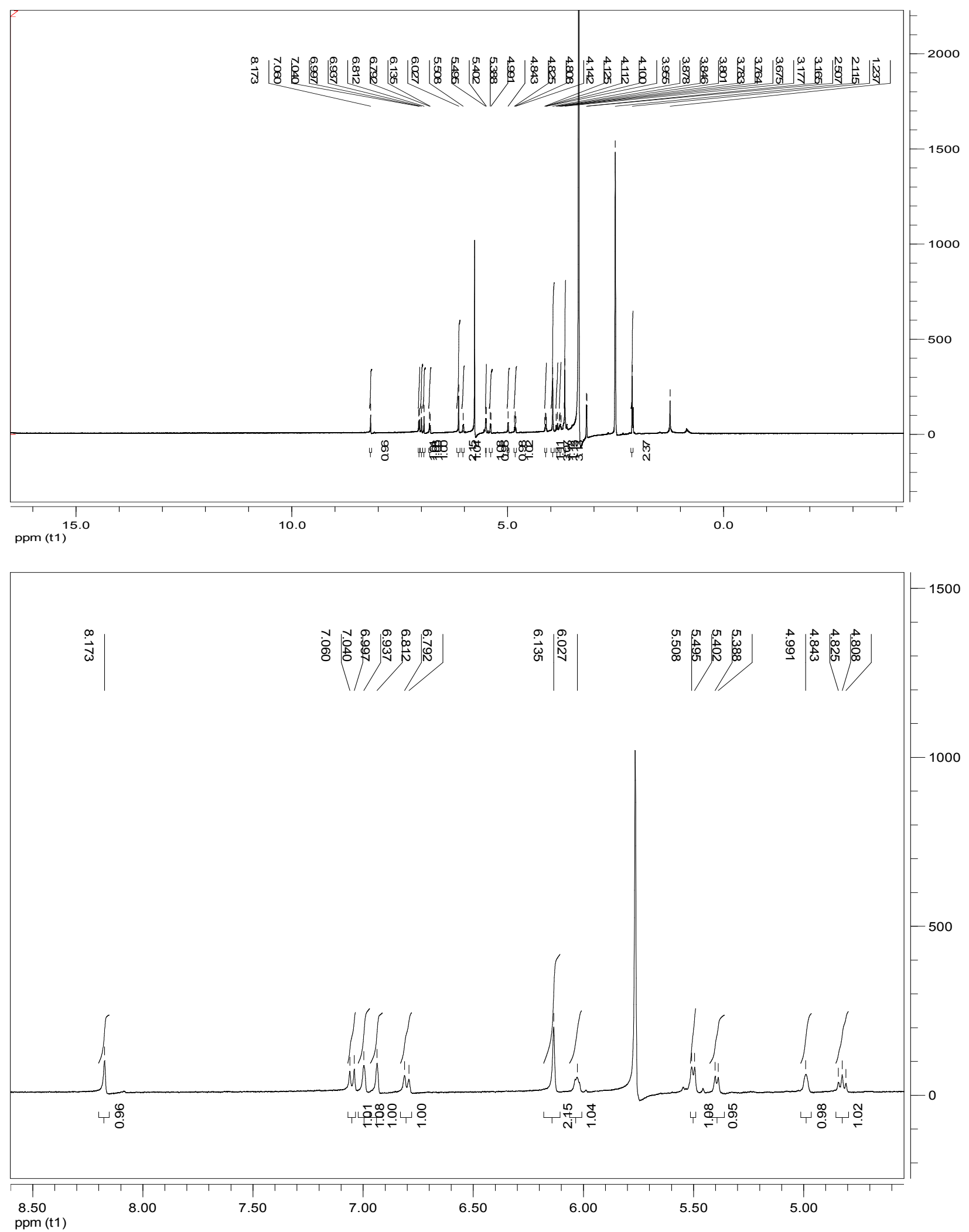


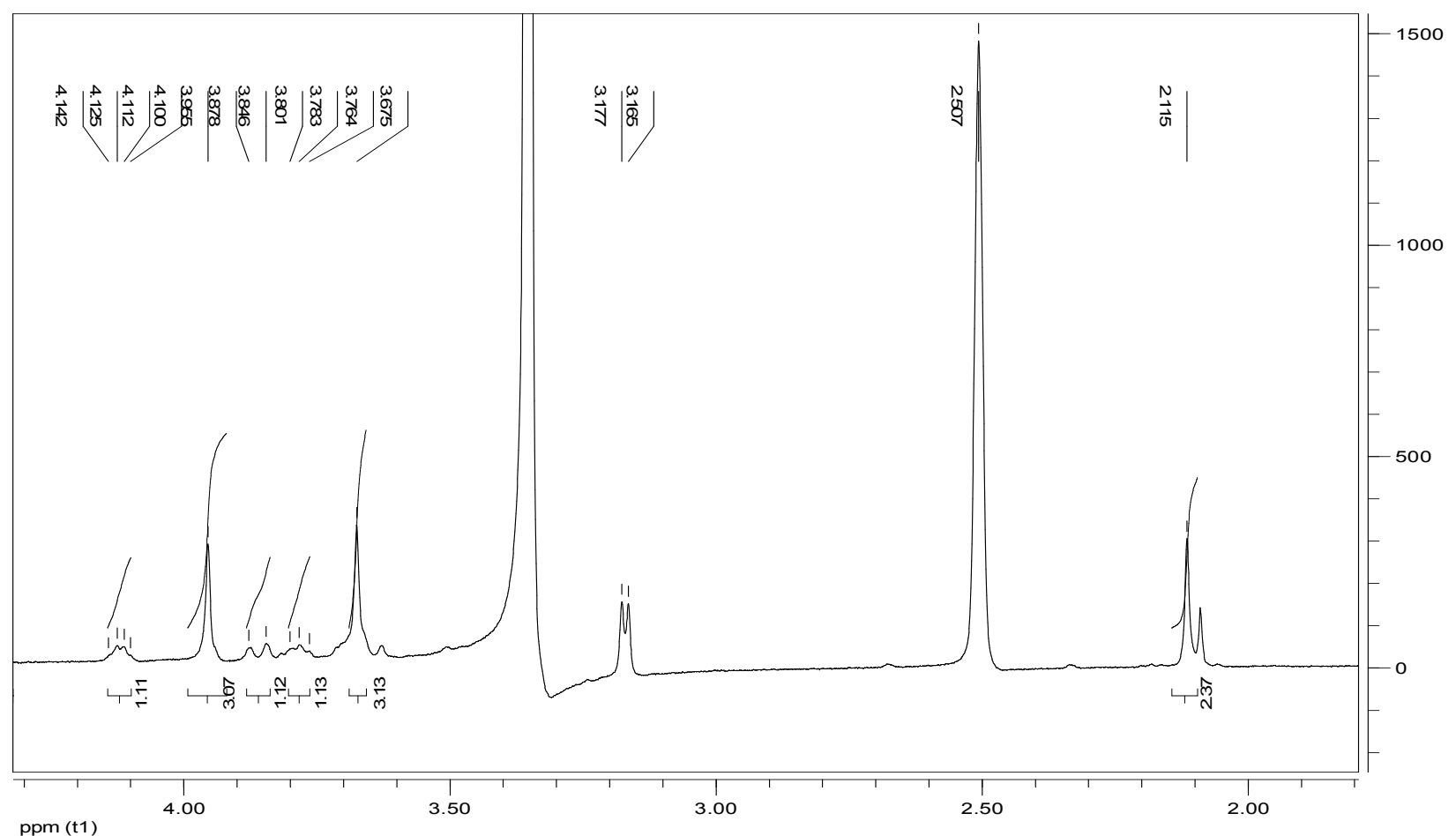

Figure S6a. ${ }^{1}$ H NMR spectrum of phyllanthusmin B (3).

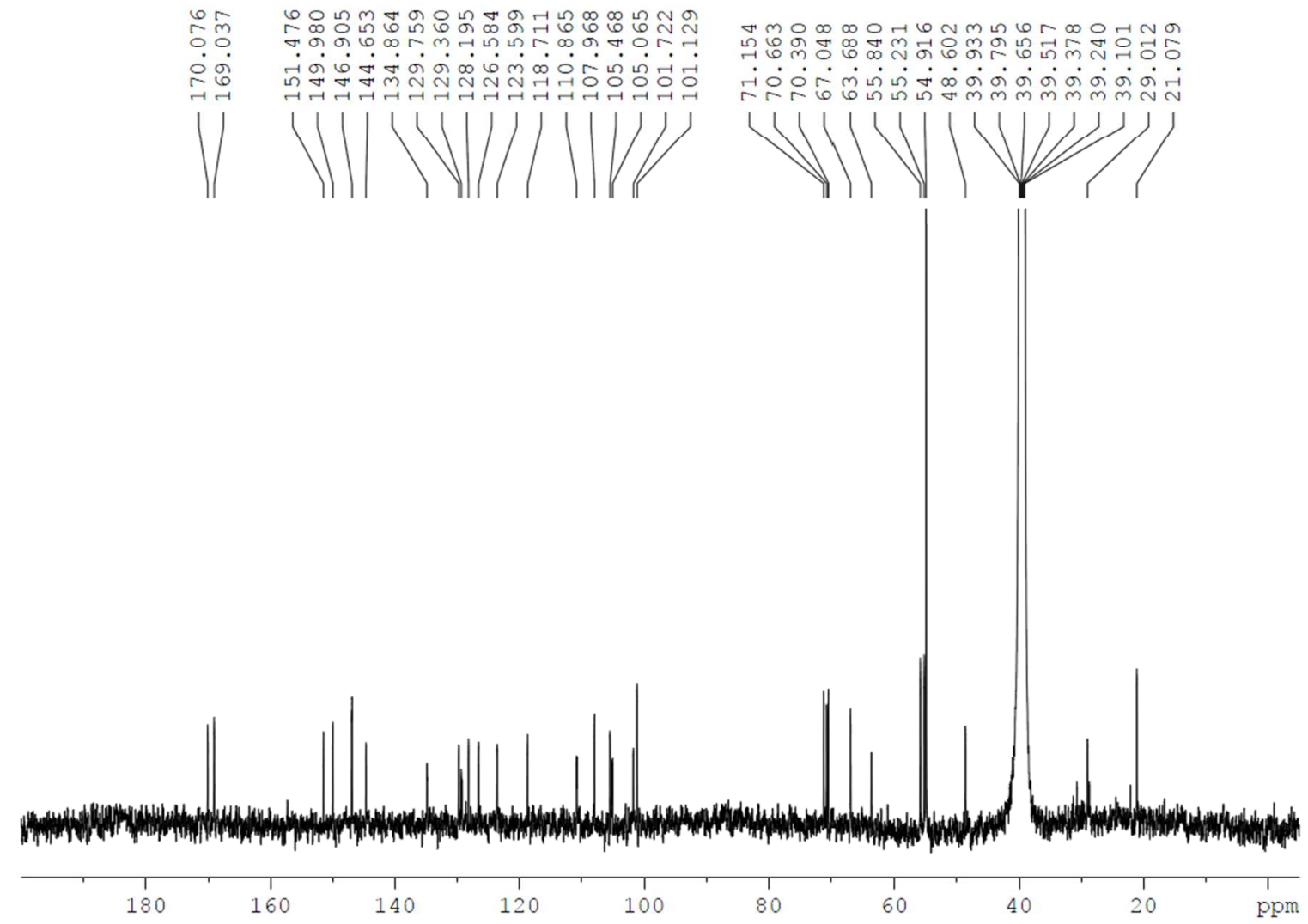



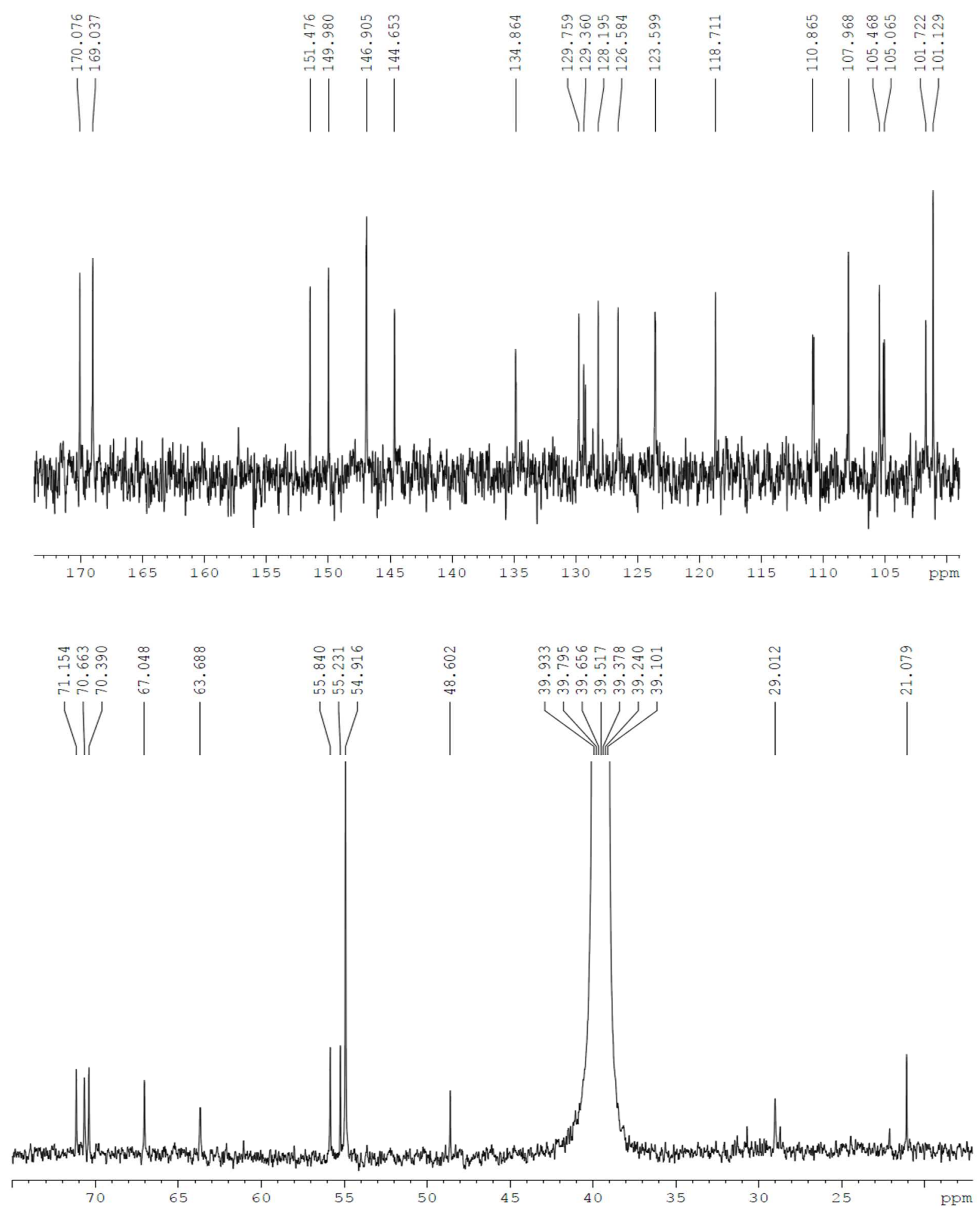

Figure S6b. ${ }^{1}$ C NMR spectrum of phyllanthusmin B (3). 


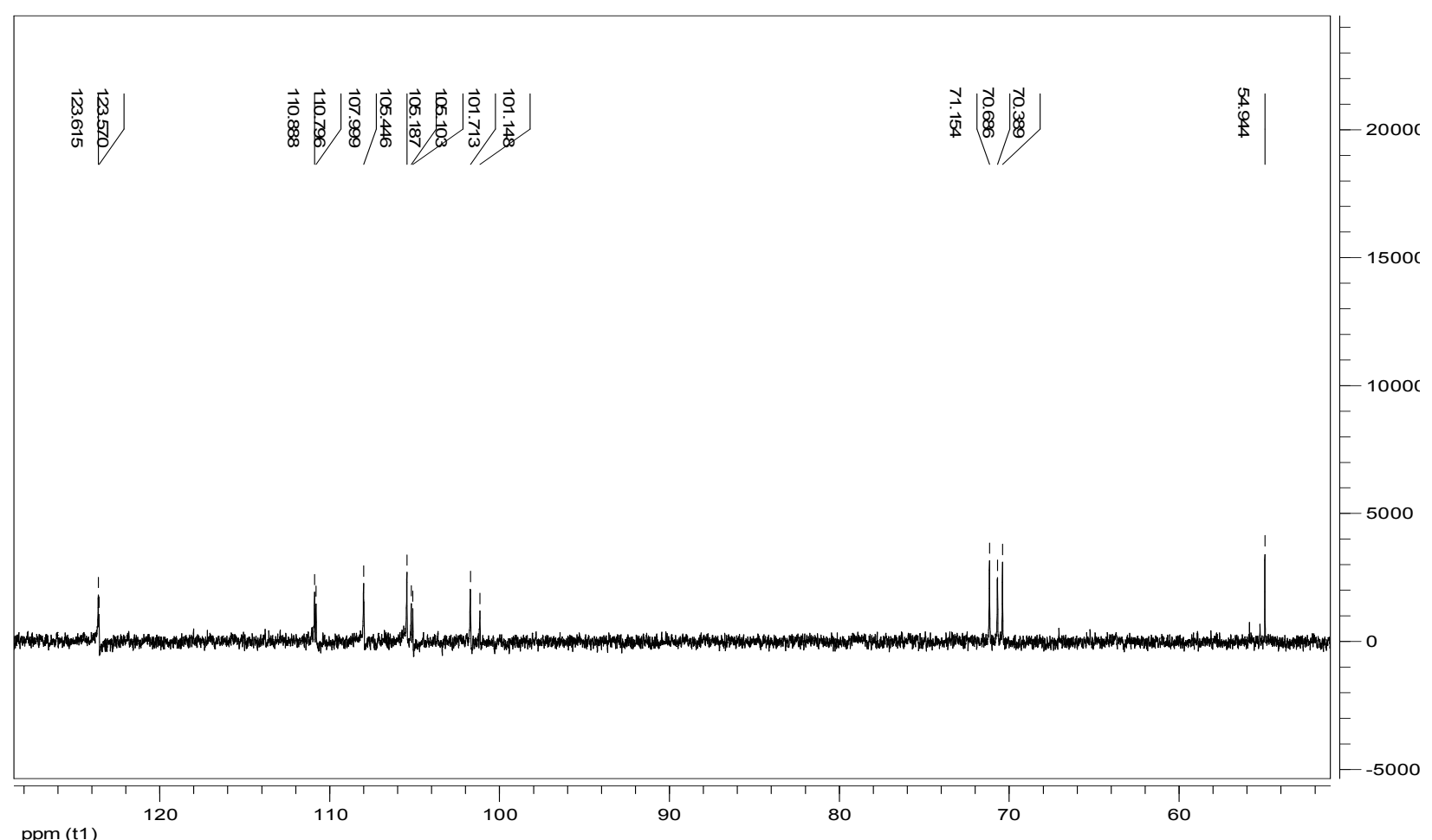

Figure S6c. DEPT 90 NMR spectrum of phyllanthusmin B (3).

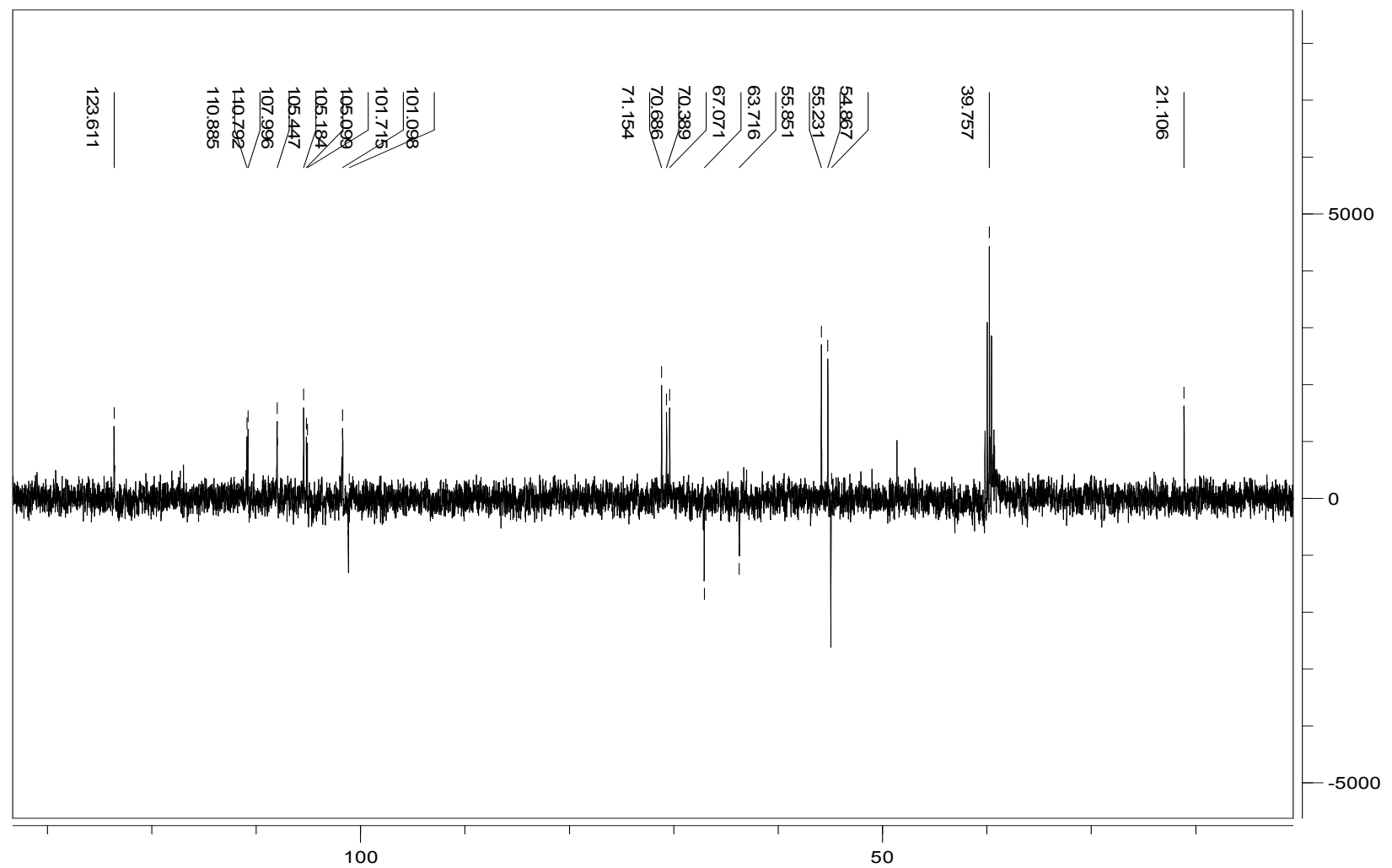

ppm (t1)

Figure S6d. DEPT 135 NMR spectrum of phyllanthusmin B (3). 


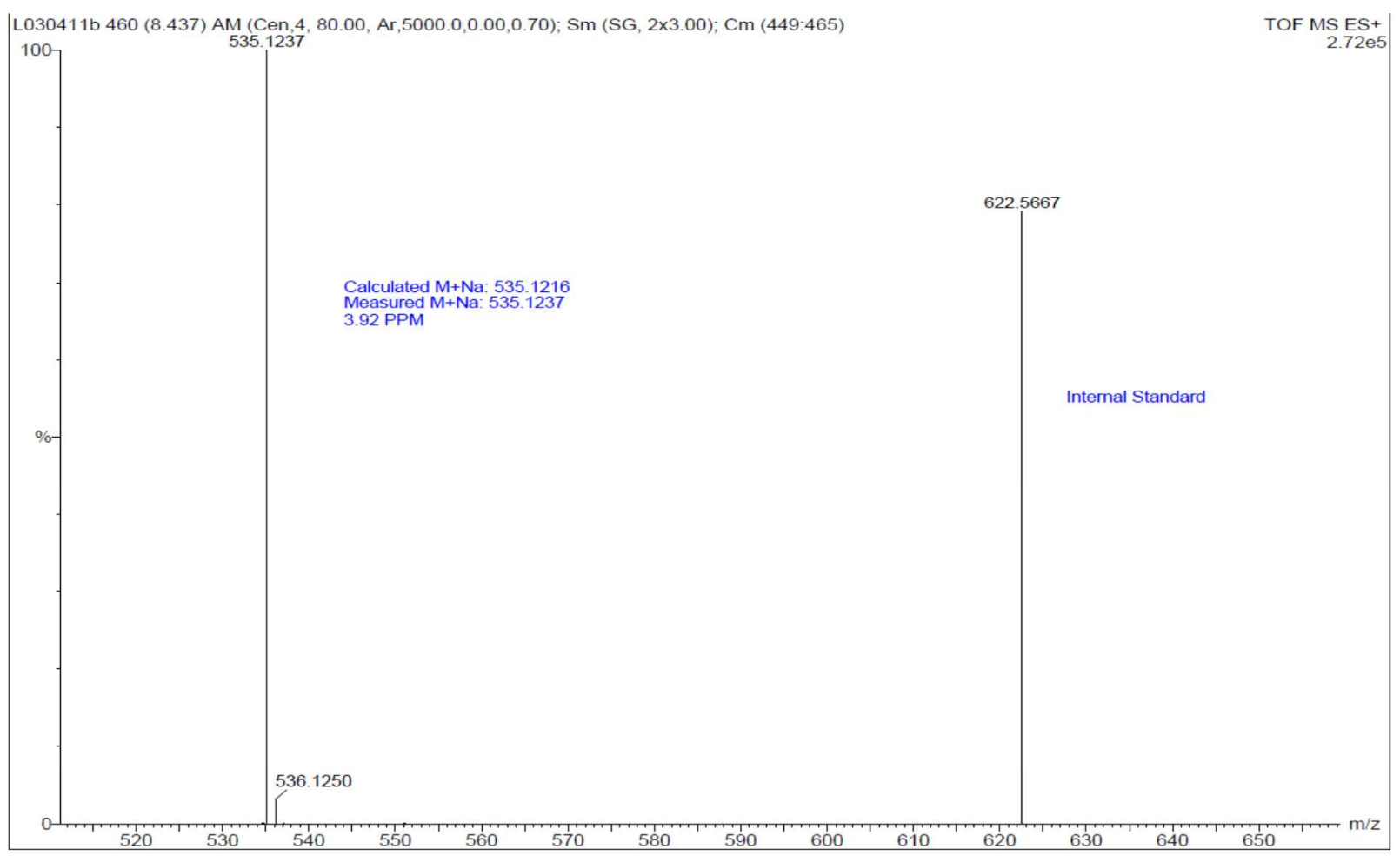

Figure S7. MS spectrum of phyllanthusmin C (4).

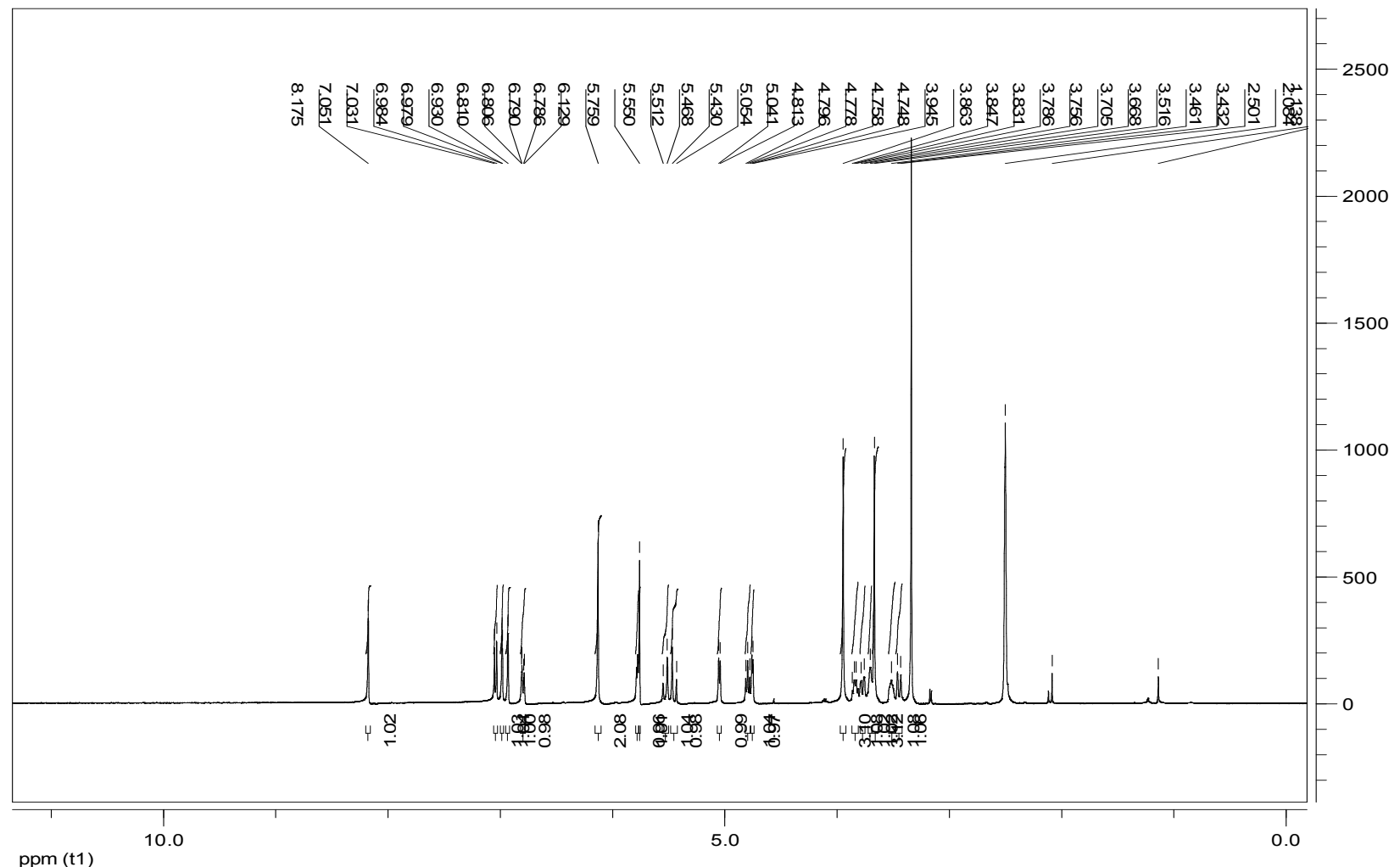



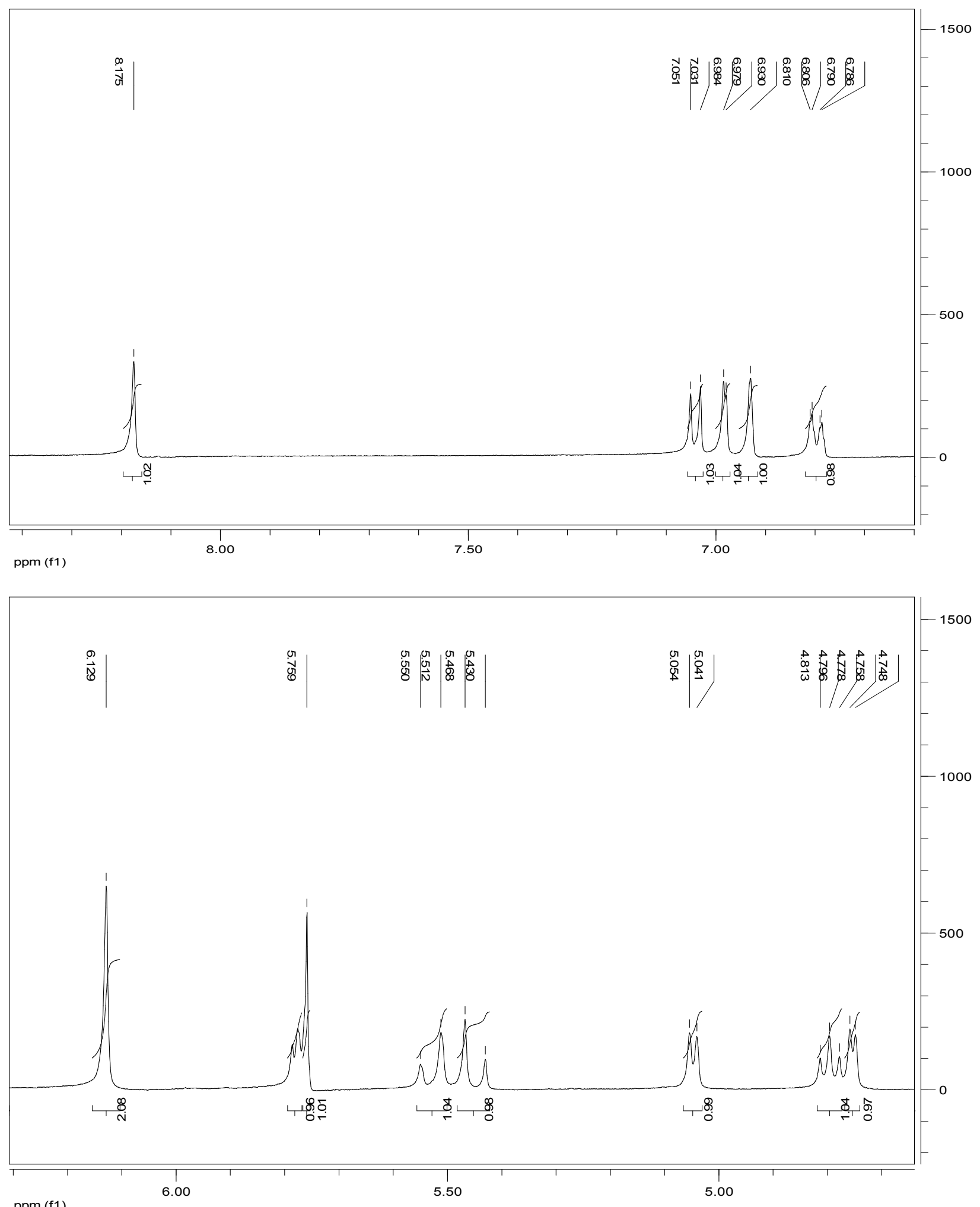


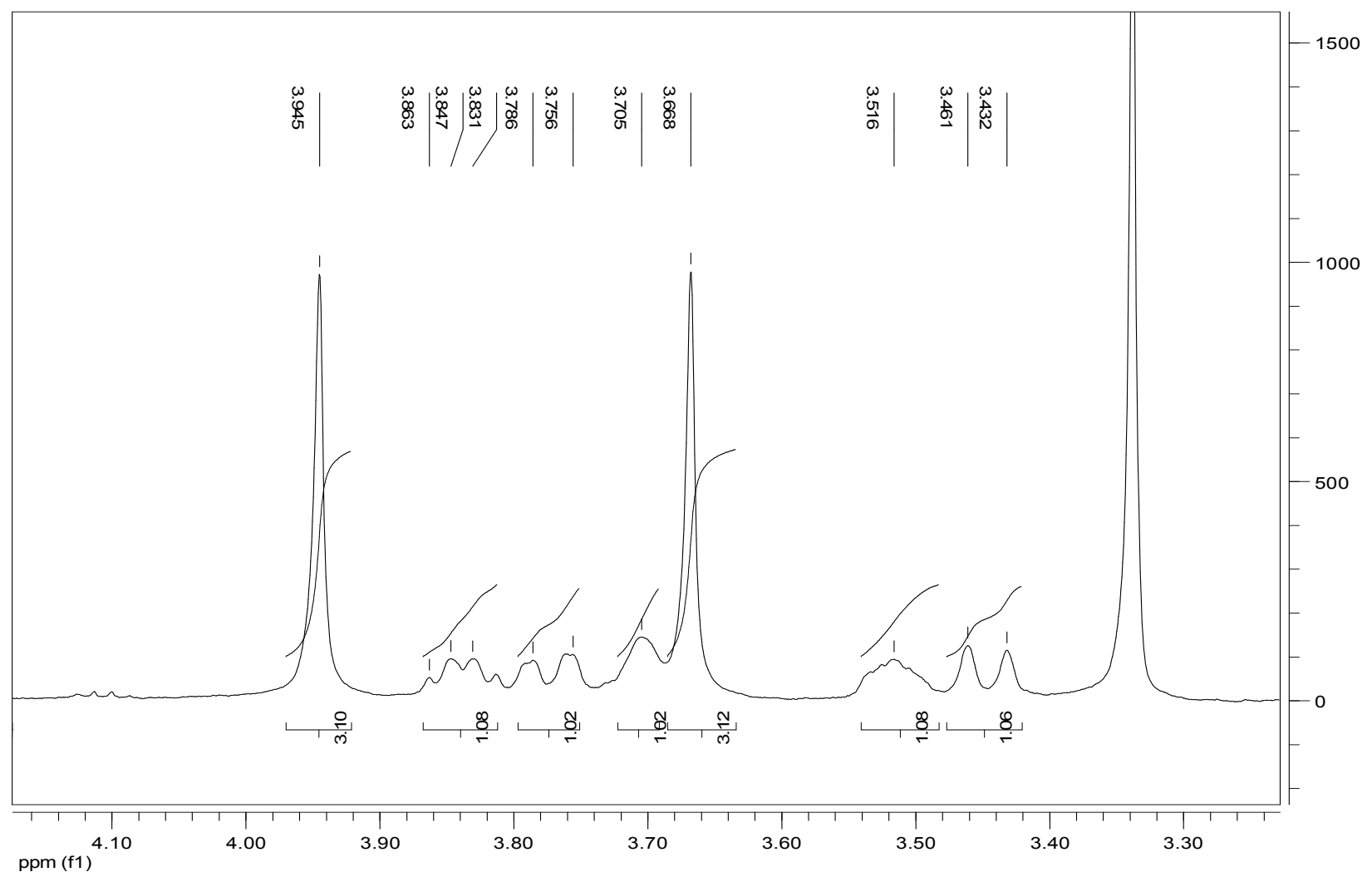

Figure S8a. ${ }^{1} \mathrm{H}$ NMR spectrum of phyllanthusmin C (4).

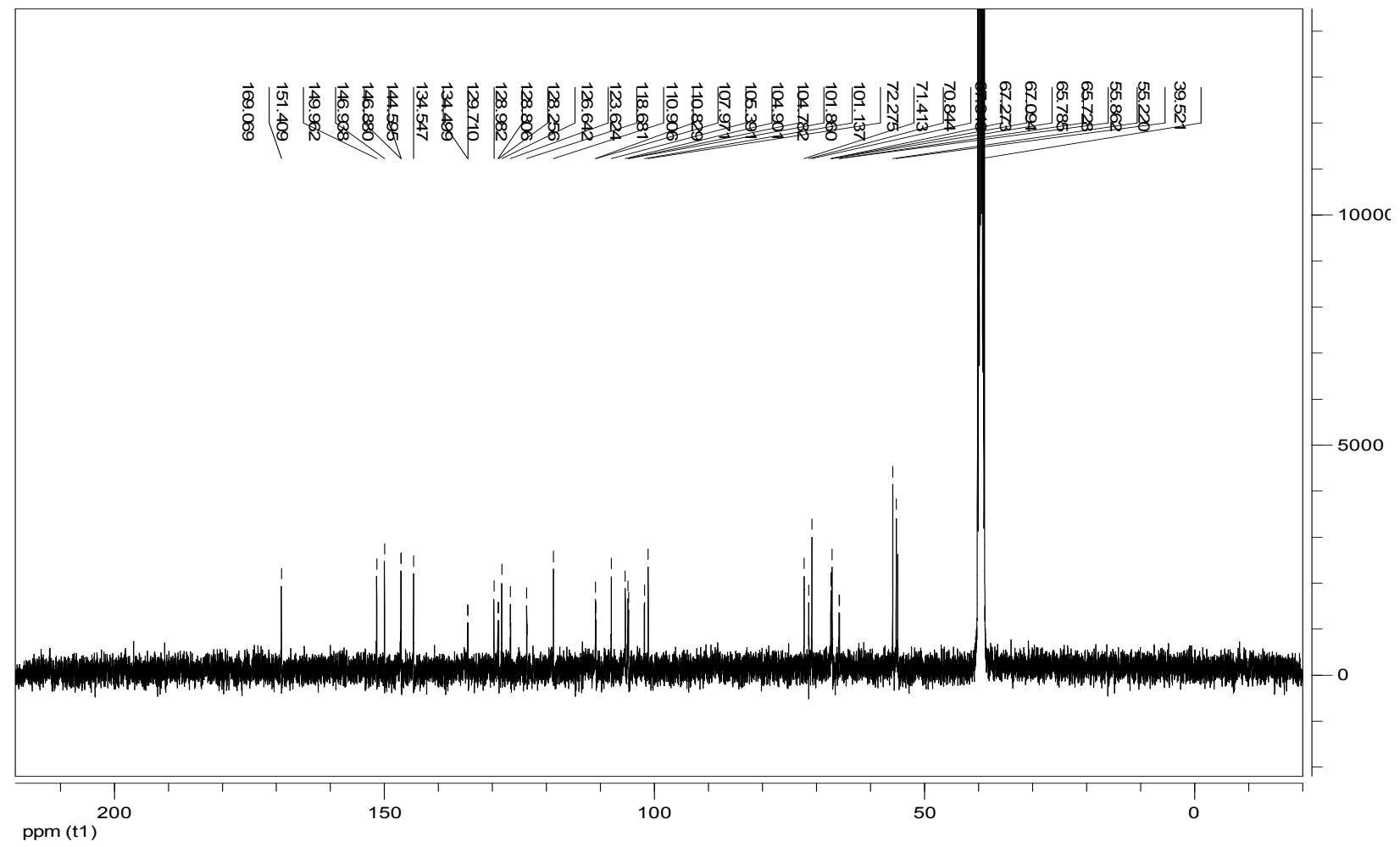




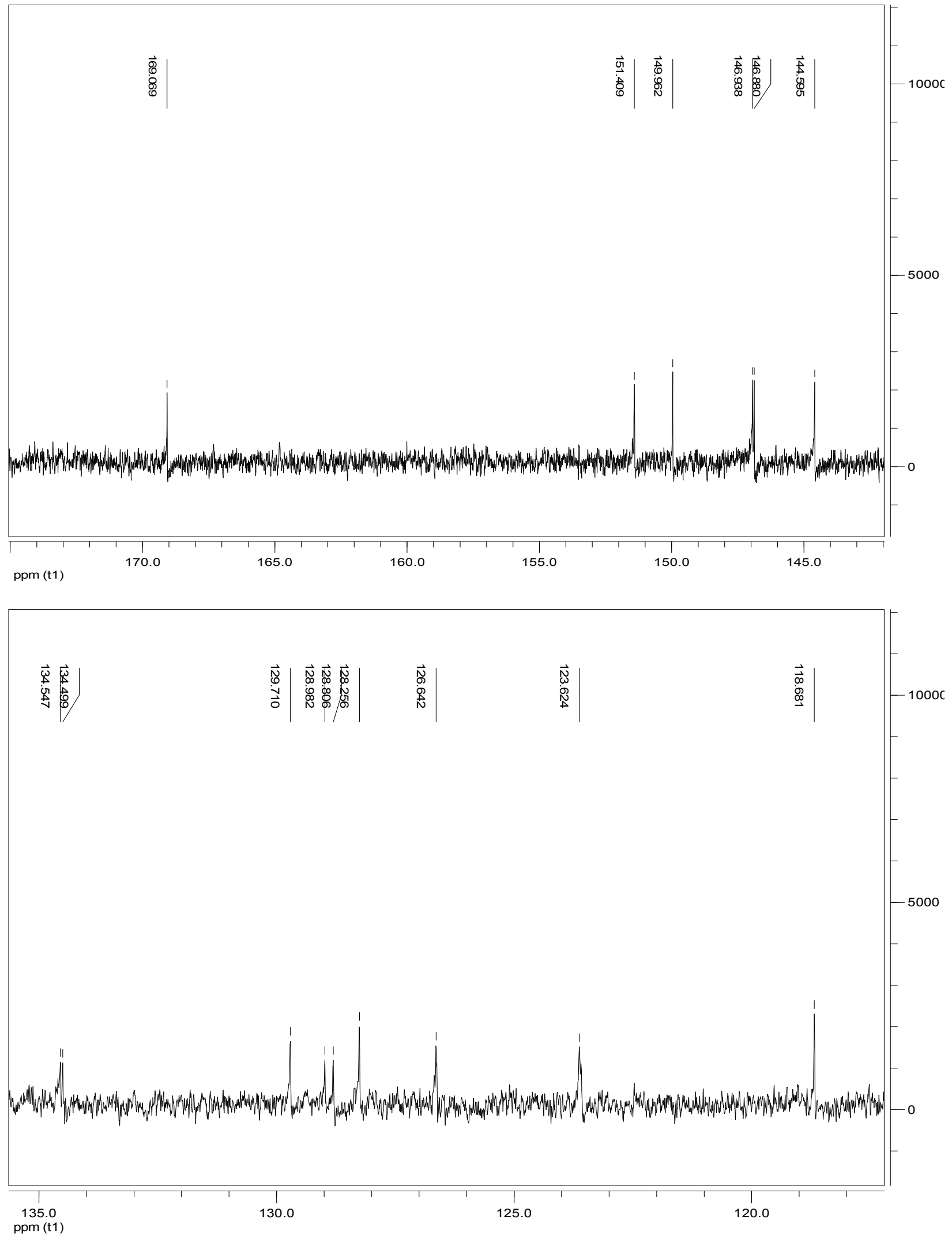




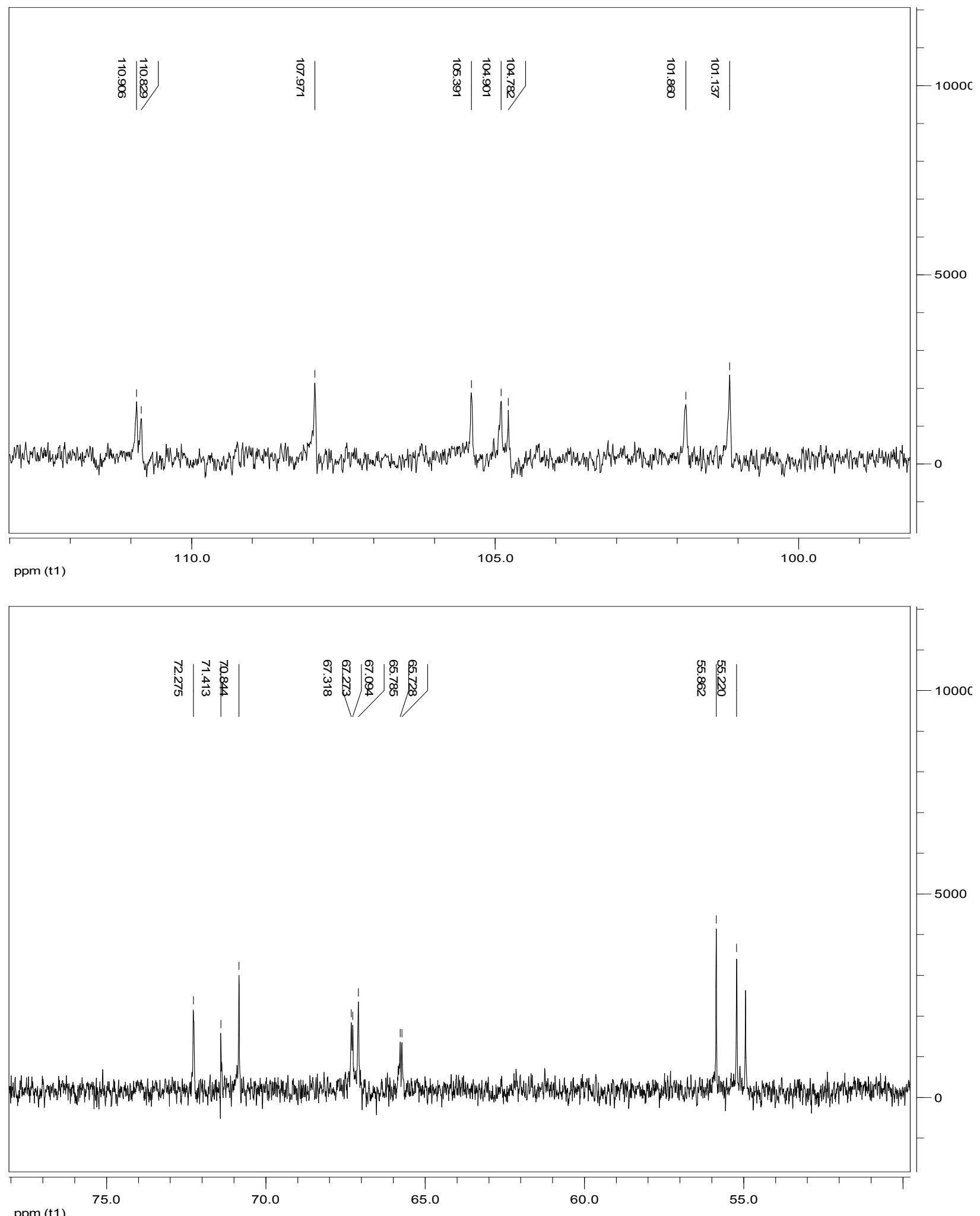

Figure S8b. ${ }^{13} \mathrm{C}$ NMR spectrum of phyllanthusmin C (4). 


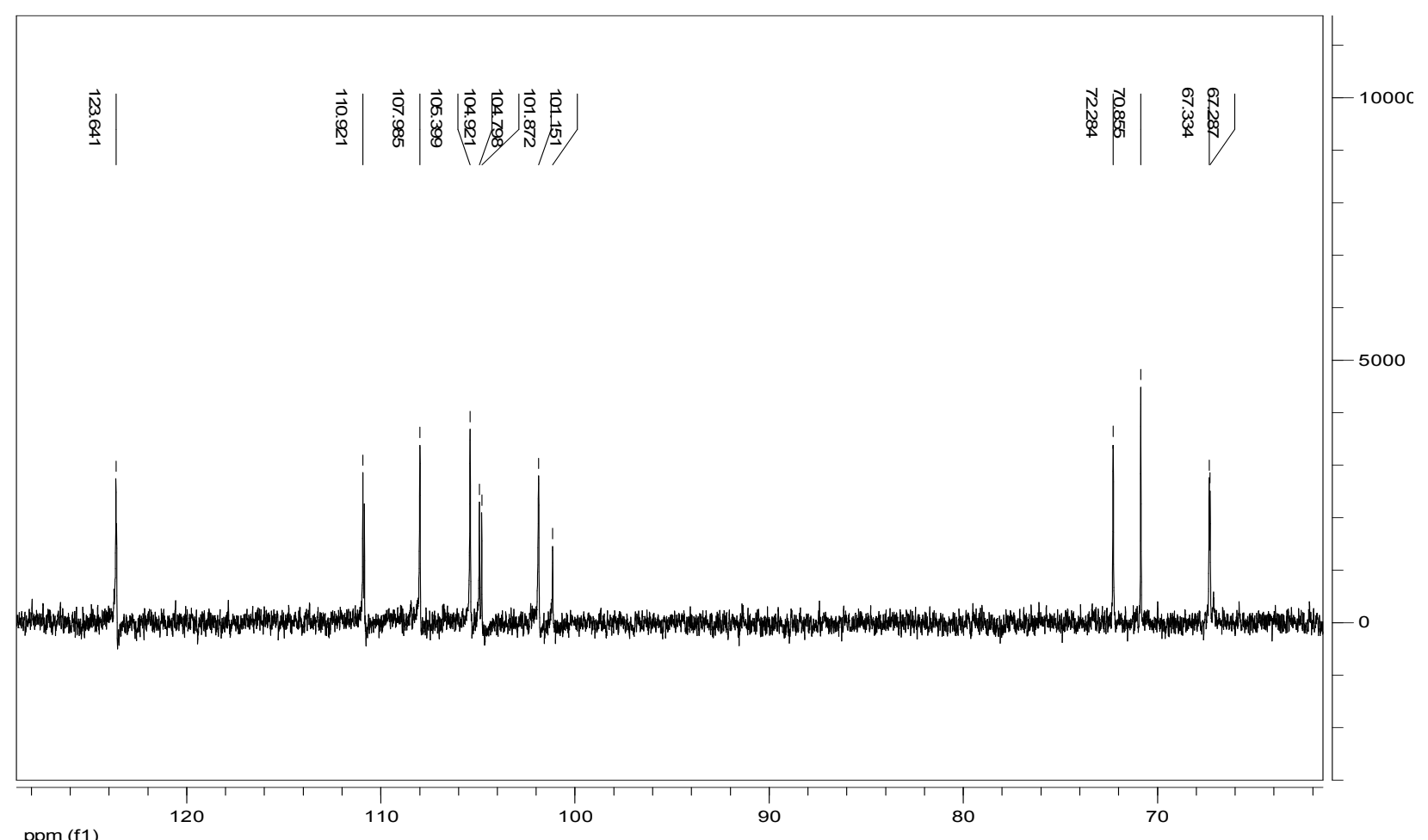

Figure S8c. DEPT 90 NMR spectrum of phyllanthusmin C (4).

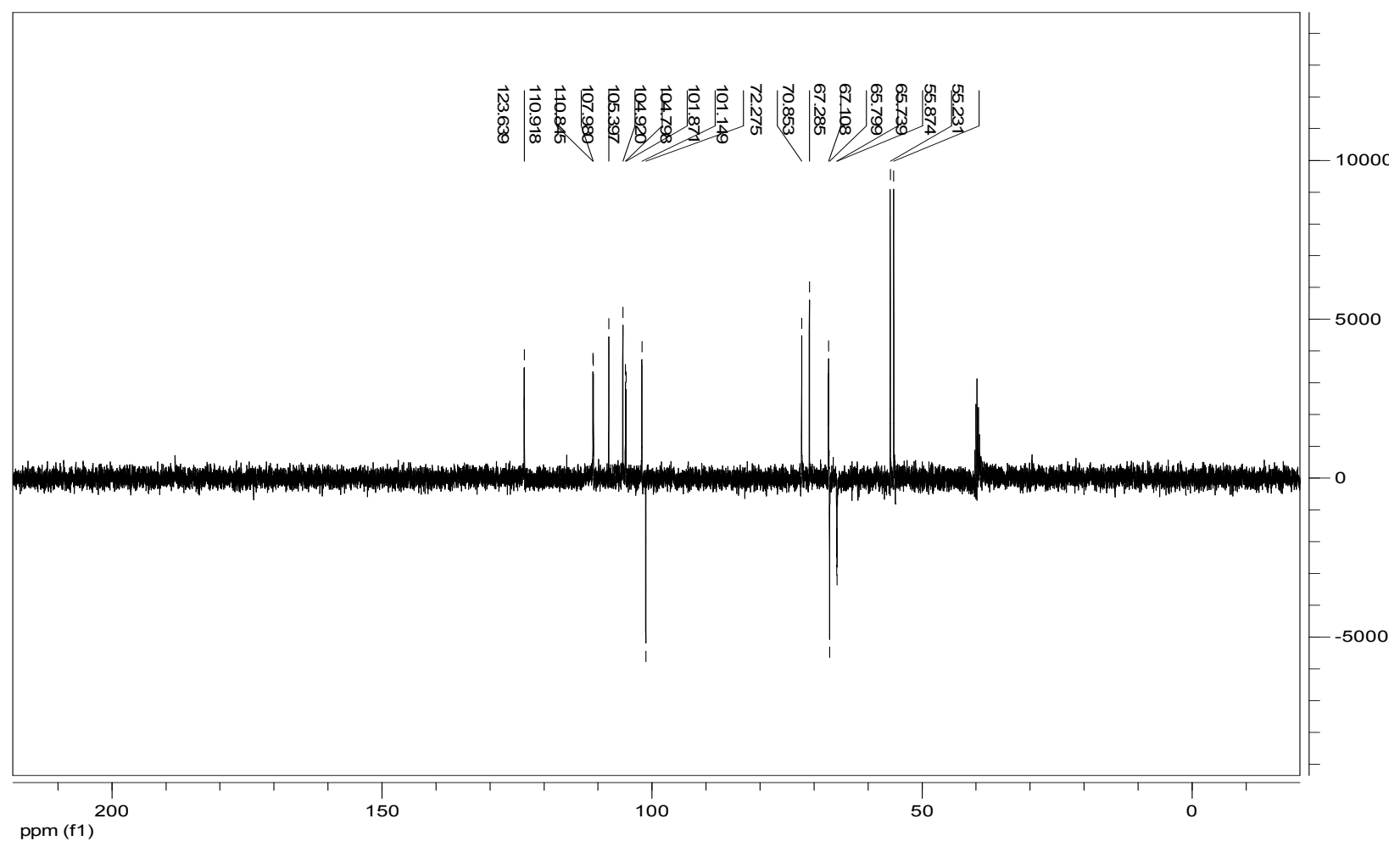

Figure S8d. DEPT 135 NMR spectrum of phyllanthusmin C (4). 


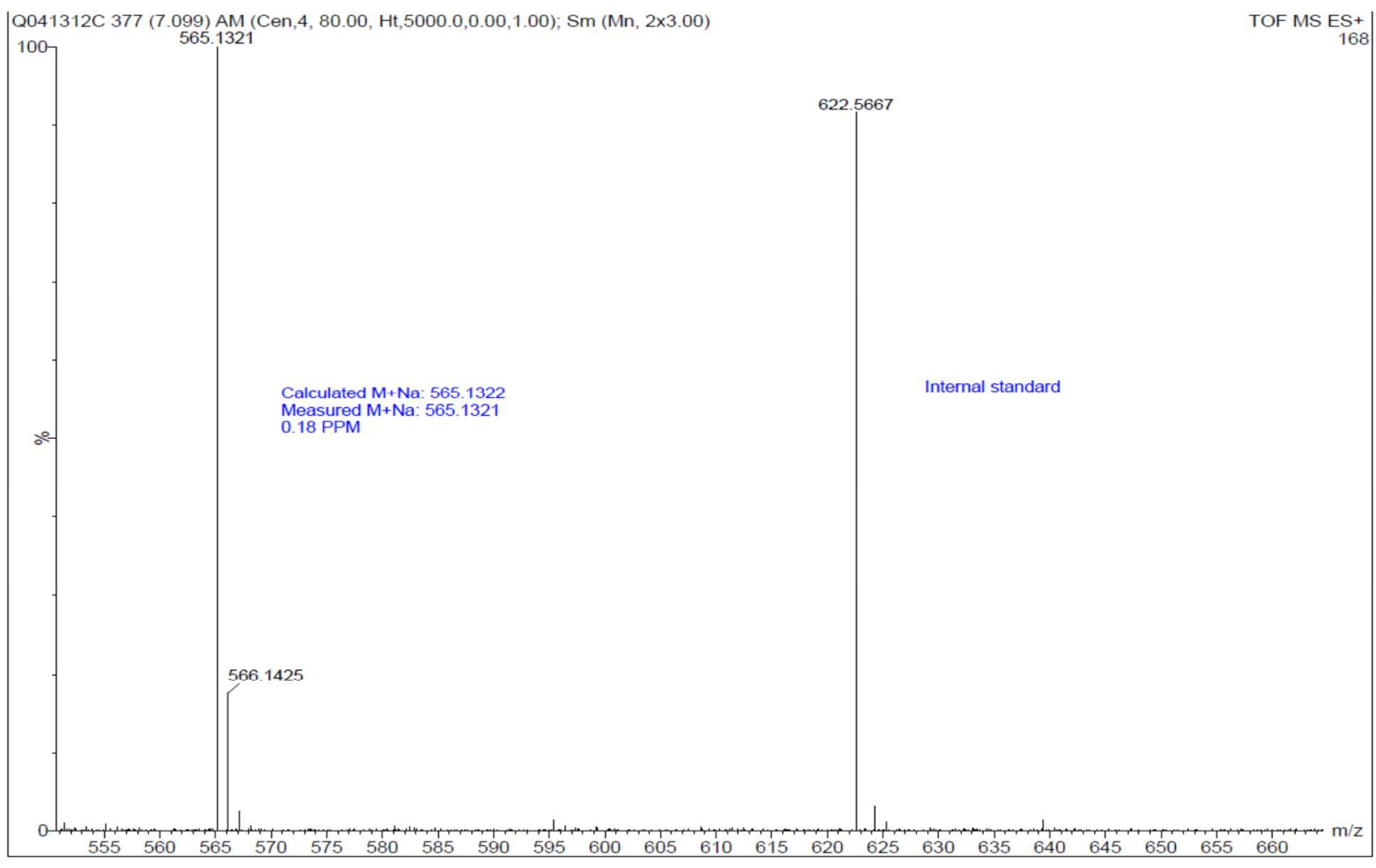

Figure S9. MS spectrum of cleistanthin B (5).

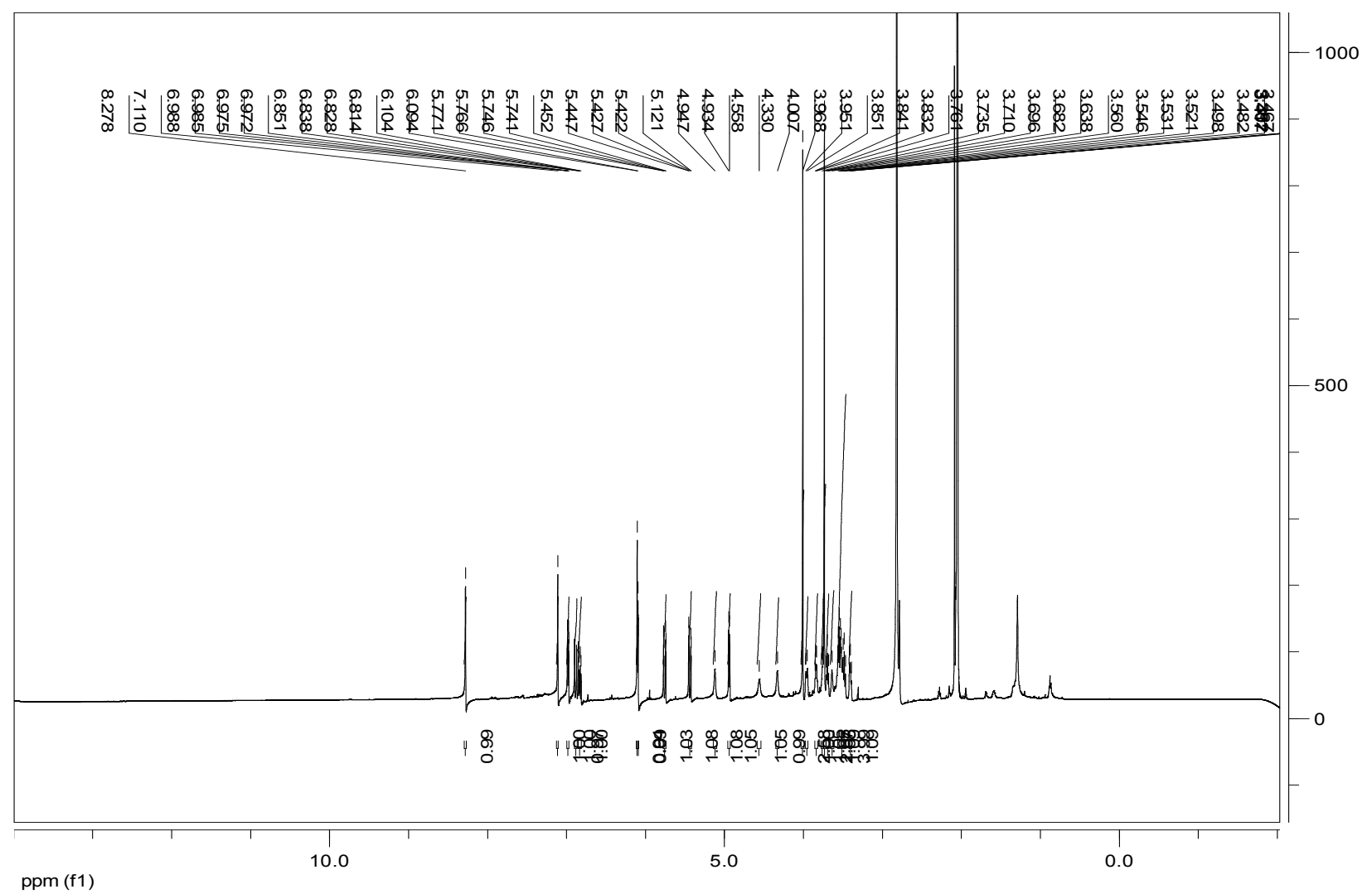



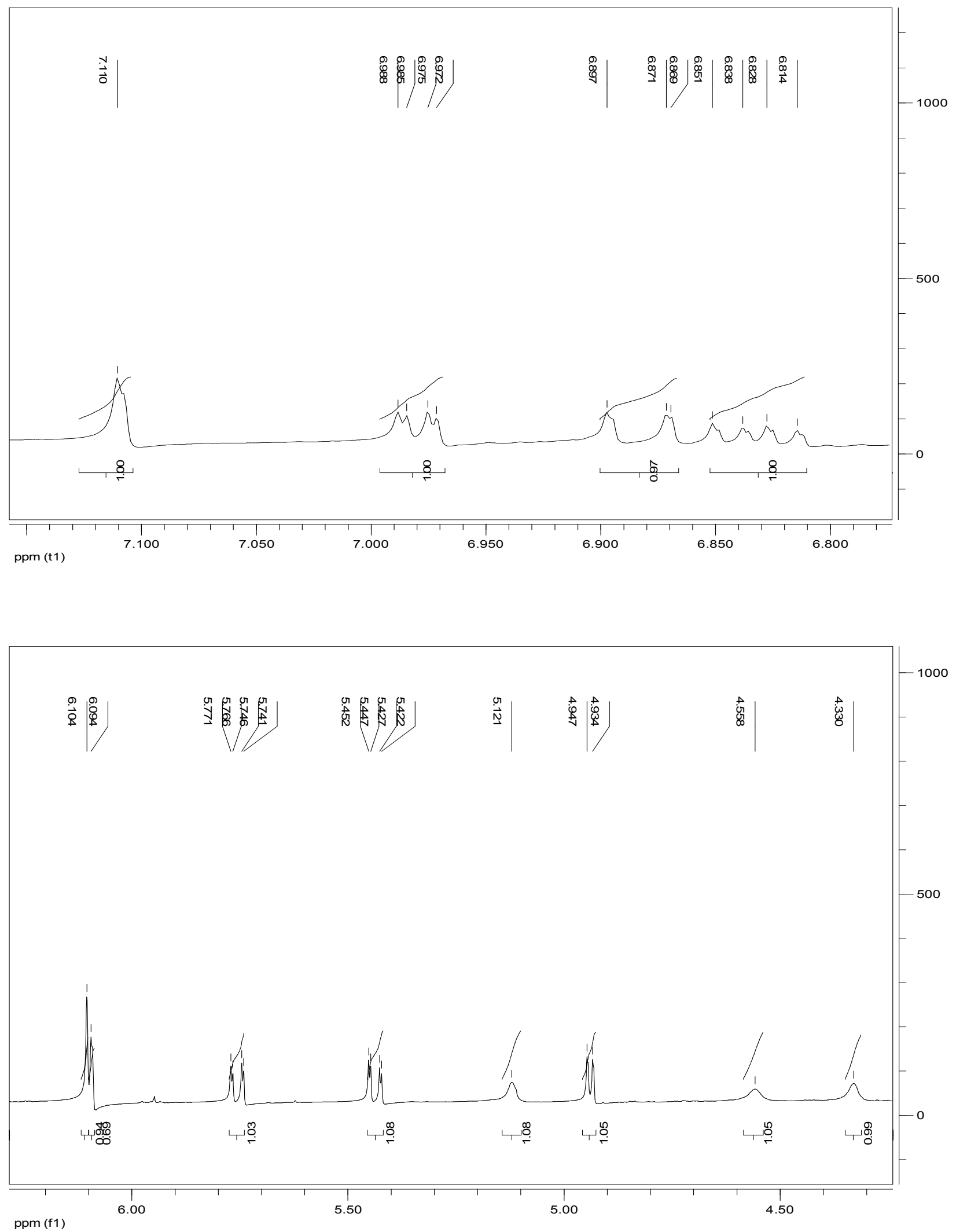

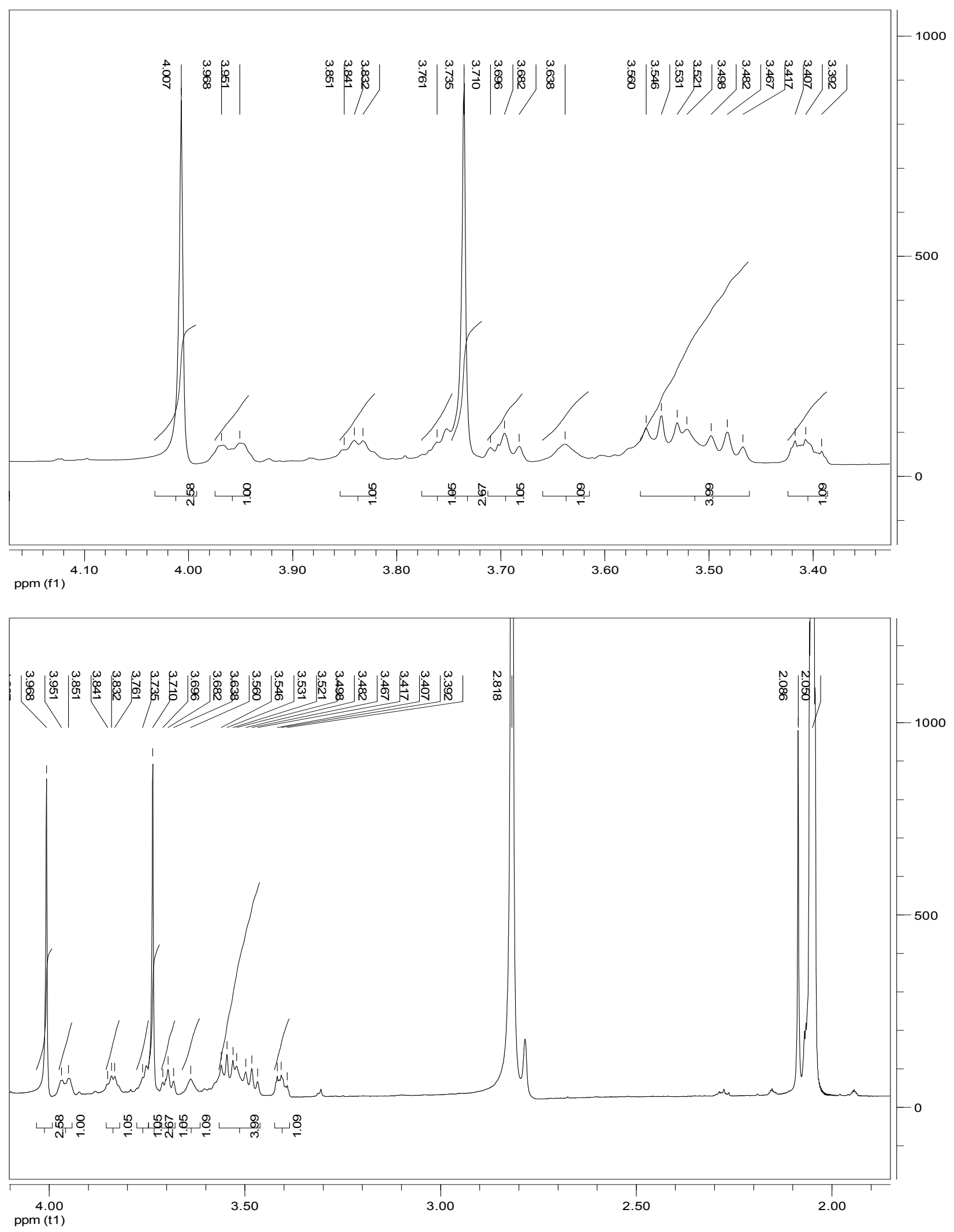

Figure S10a. ${ }^{1} \mathrm{H}$ NMR spectrum of cleistanthin B (5). 


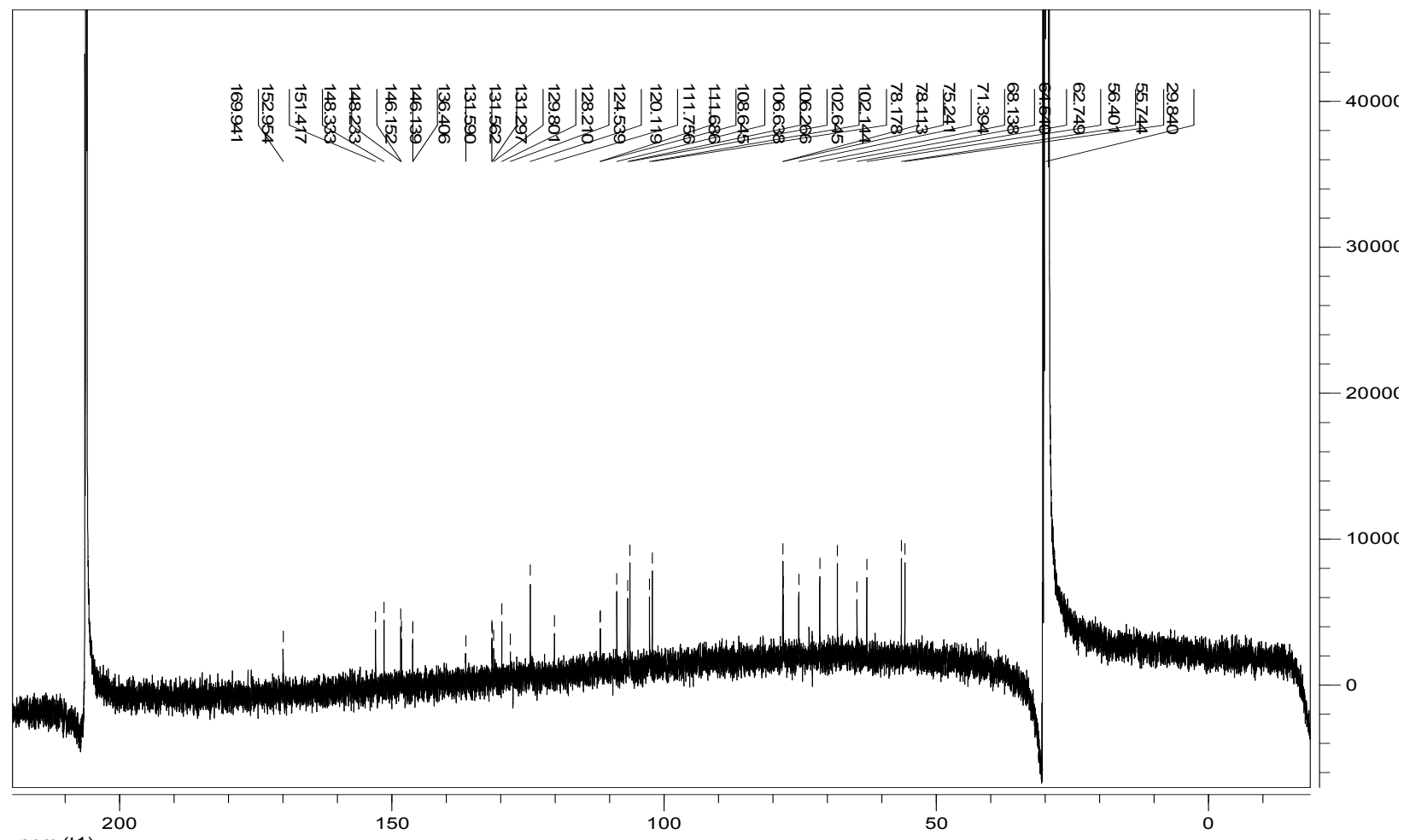

ppm (t1)

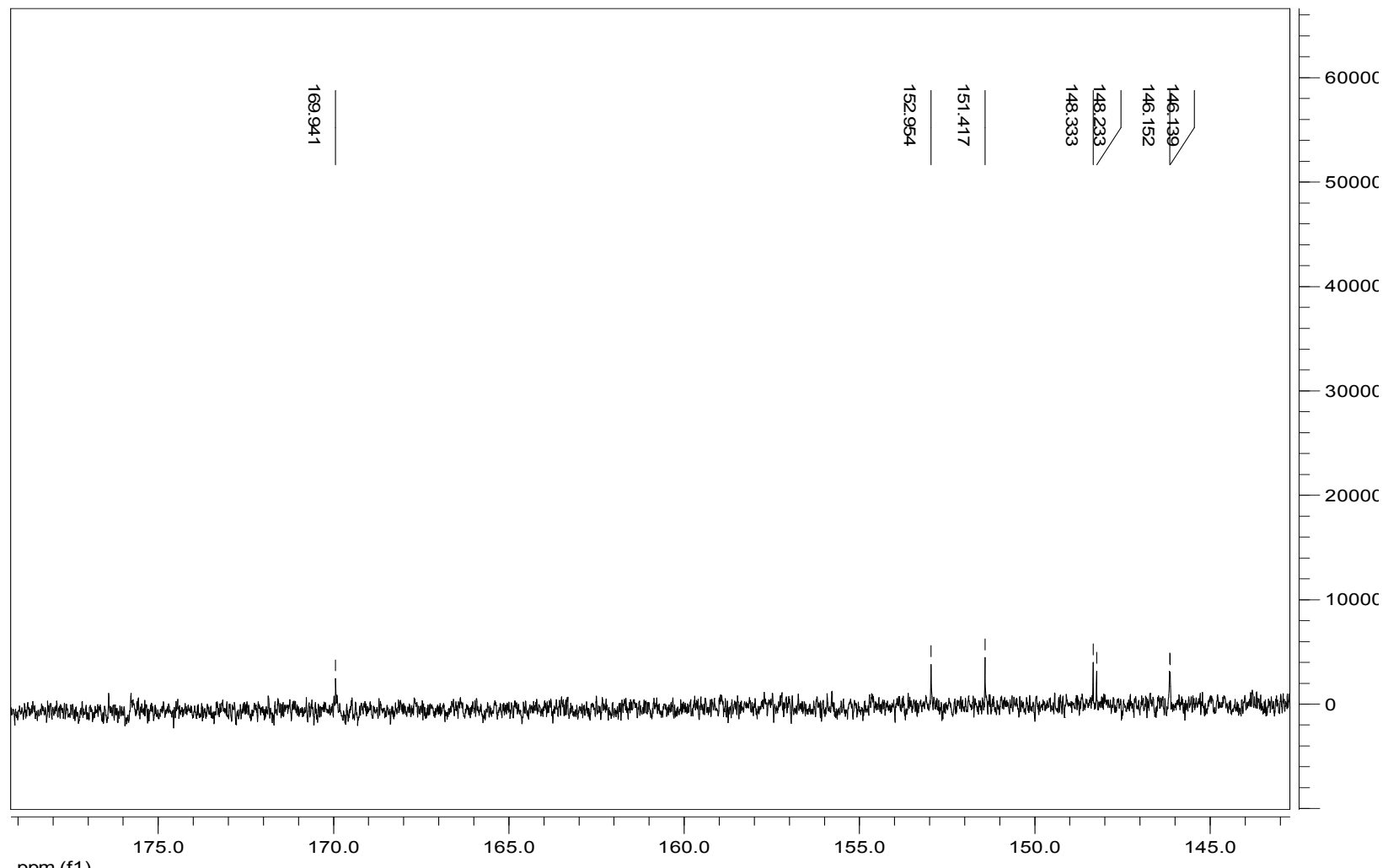




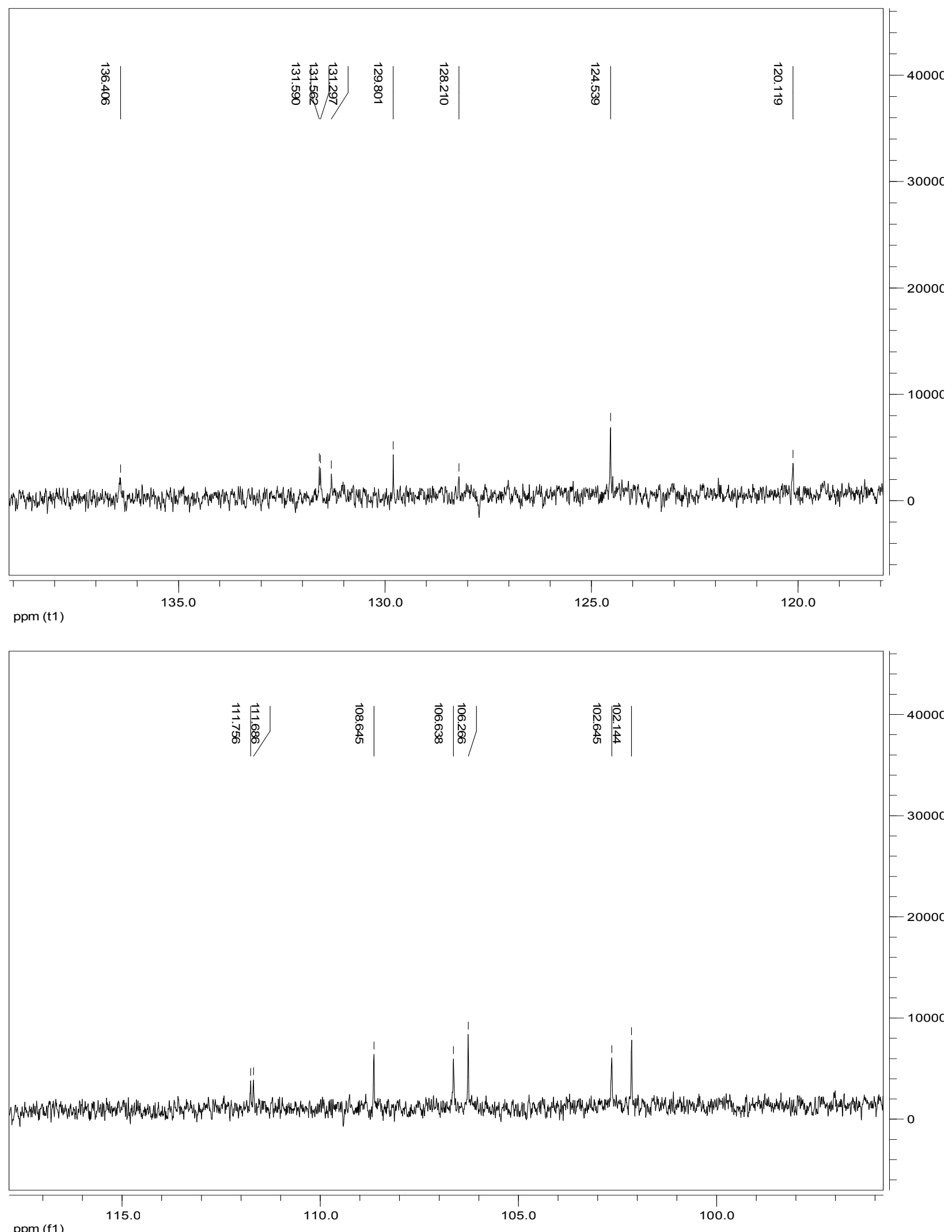




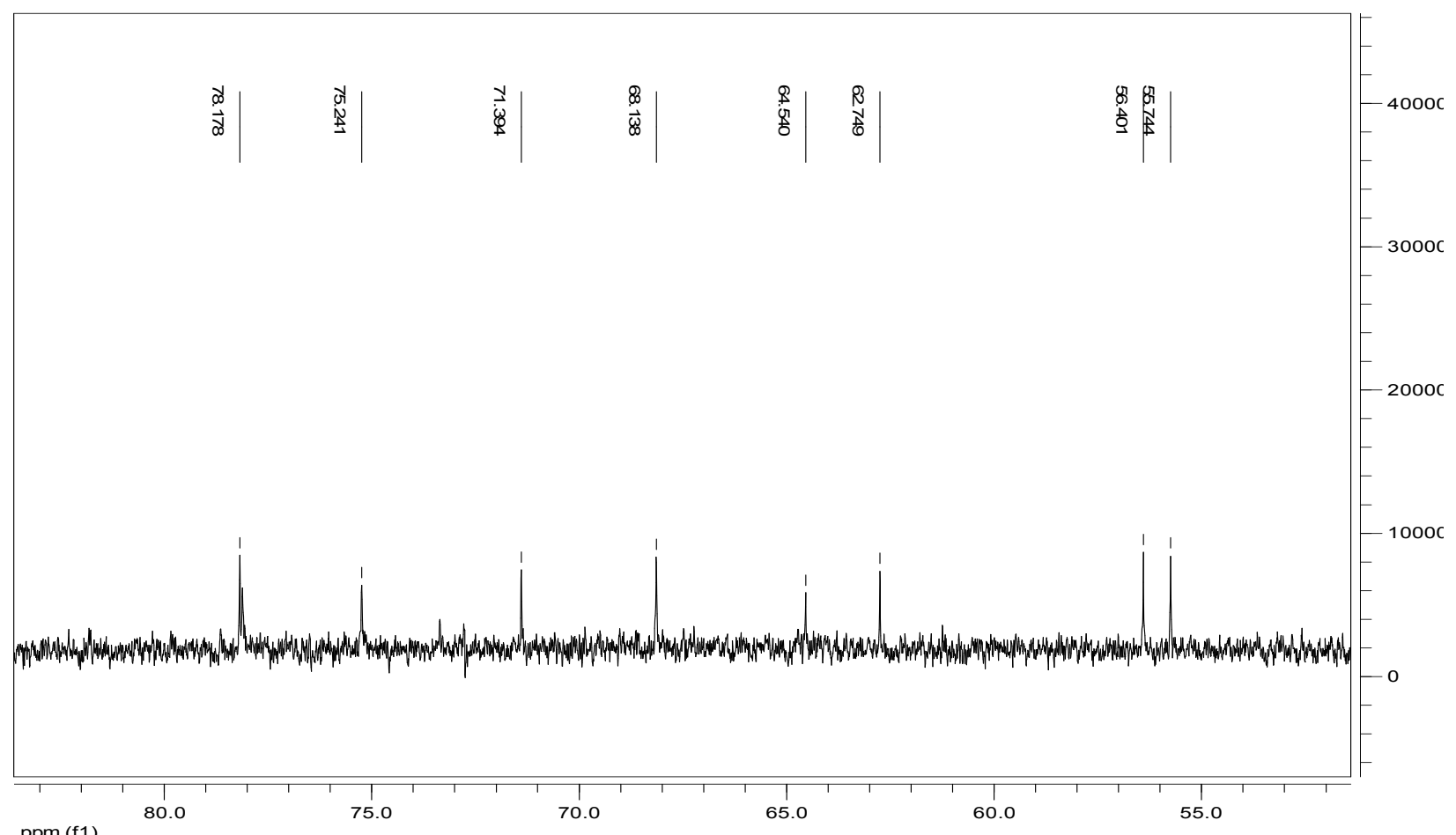

Figure S10b. ${ }^{13} \mathrm{C}$ NMR spectrum of cleistanthin B (5).

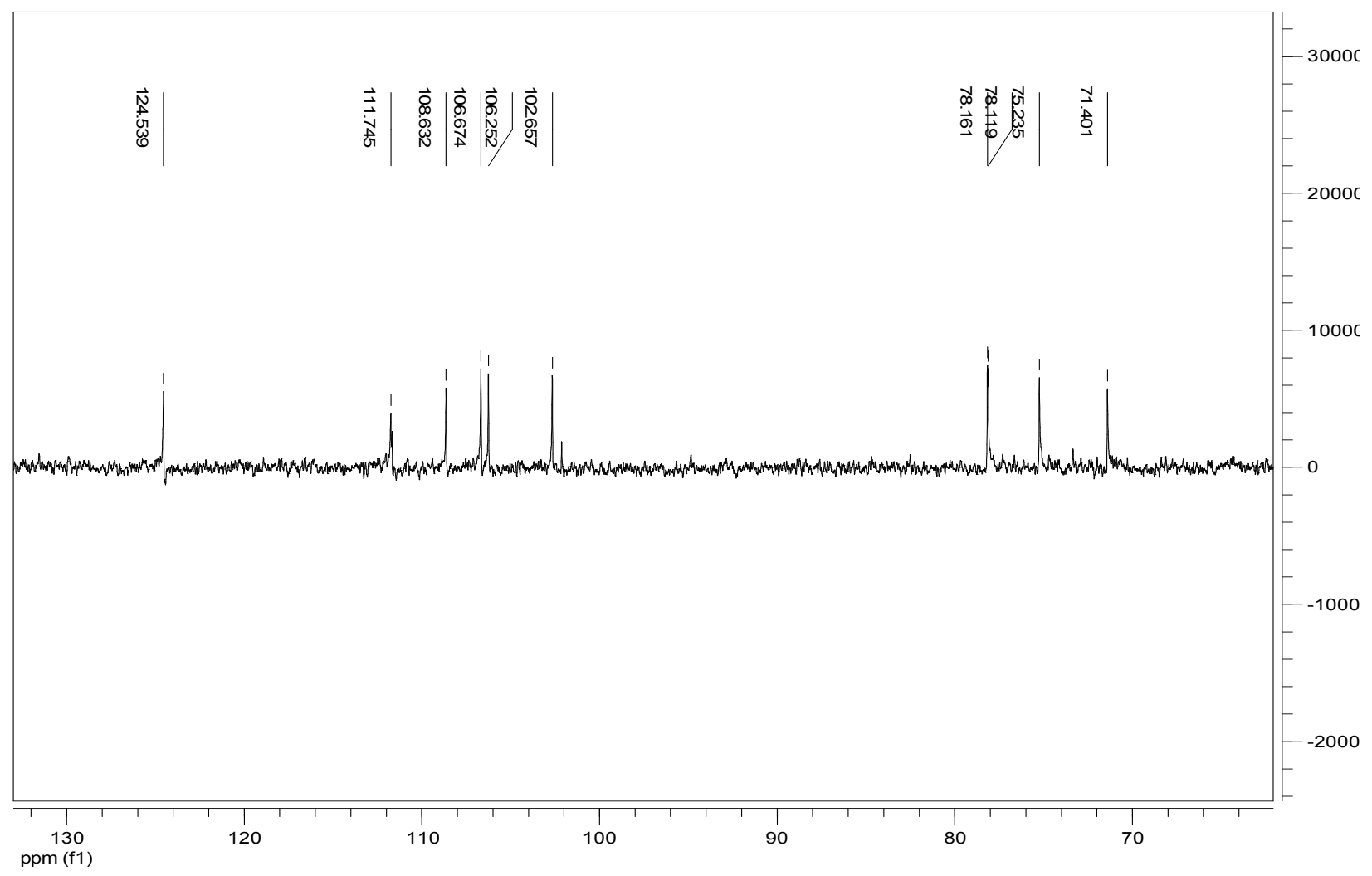

Figure S10c. DEPT 90 NMR spectrum of cleistanthin B (5). 


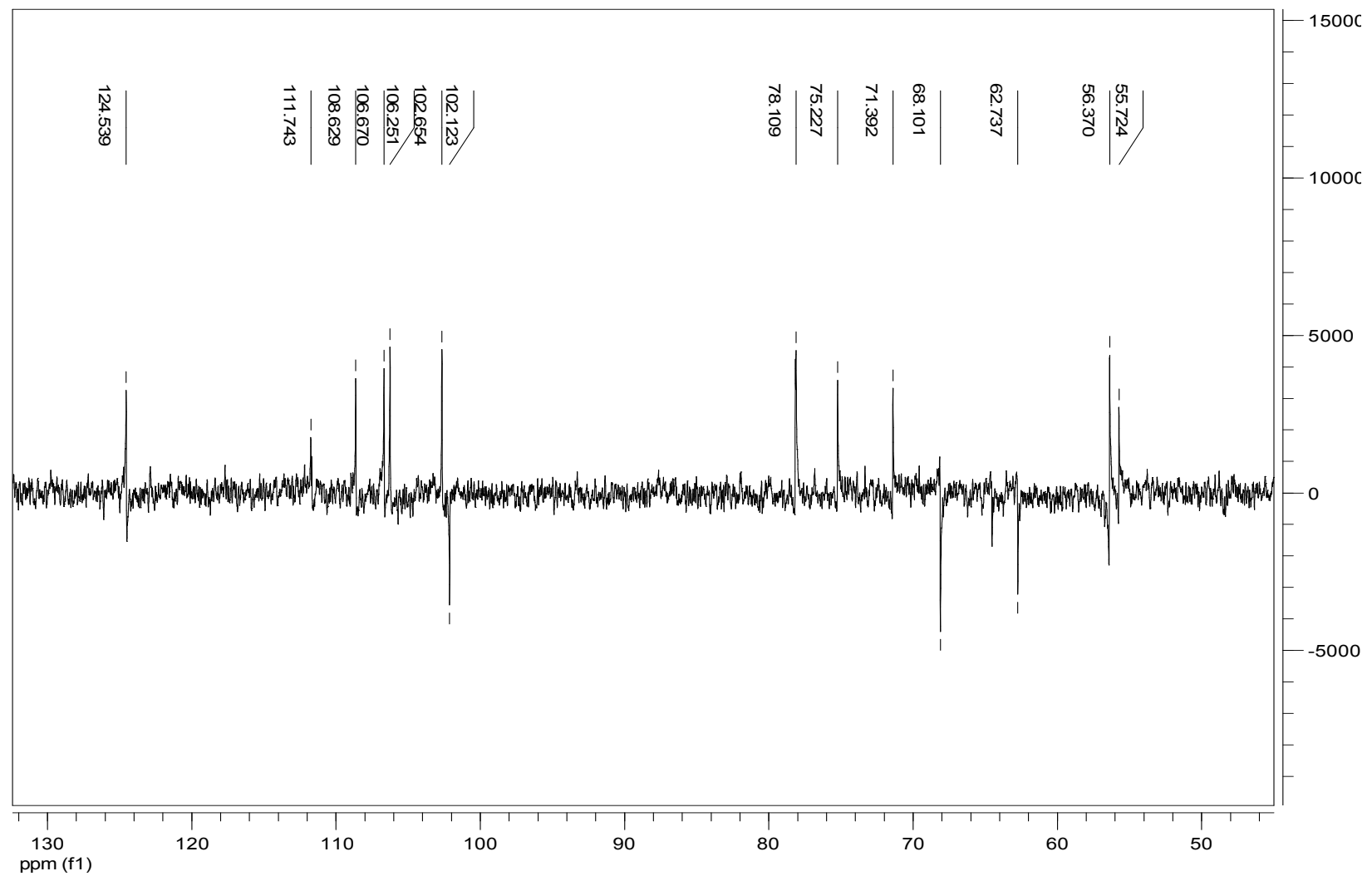

Figure S10d. DEPT 135 NMR spectrum of cleistanthin B (5).

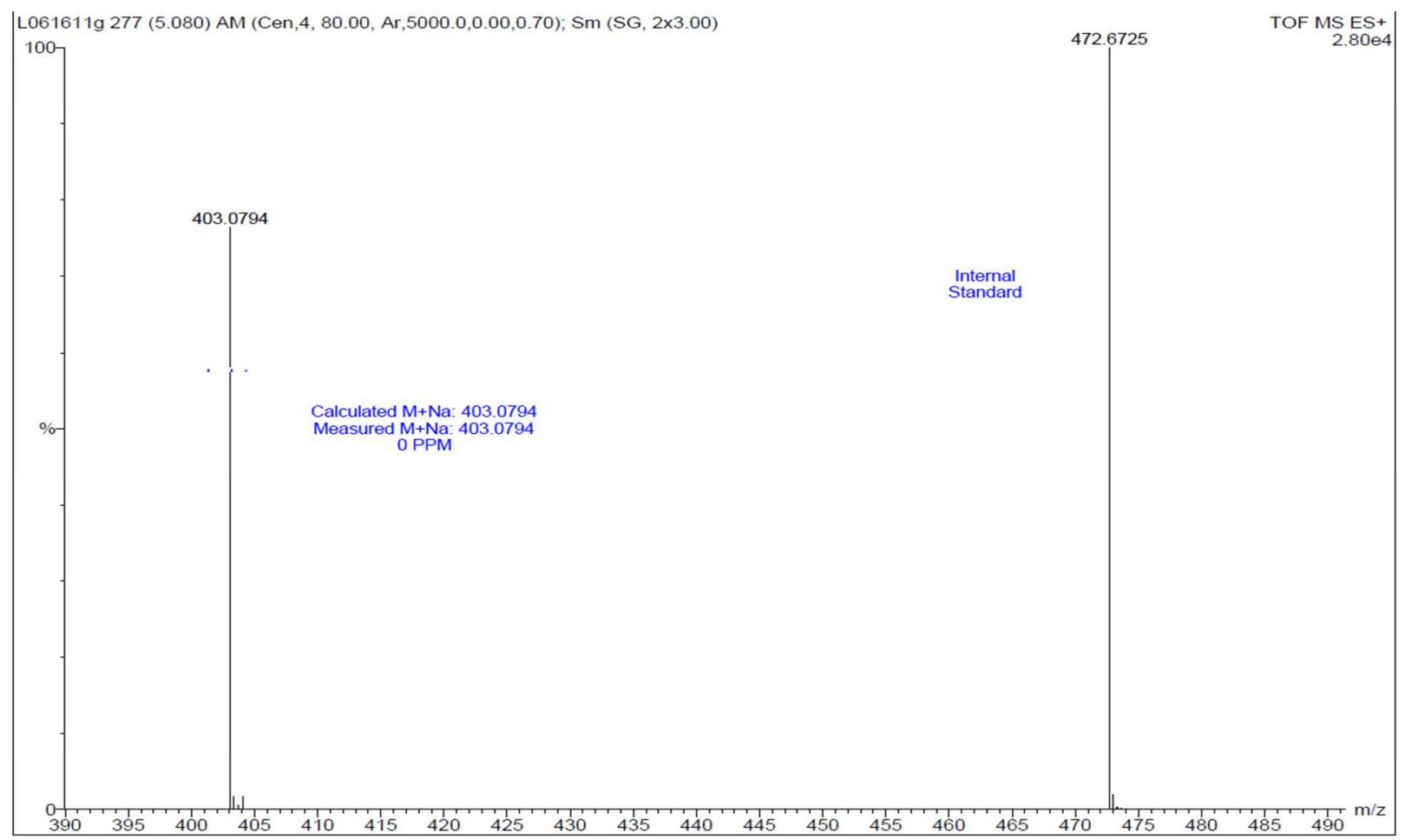

Figure S11. MS spectrum of phyllanthusmin A (6). 

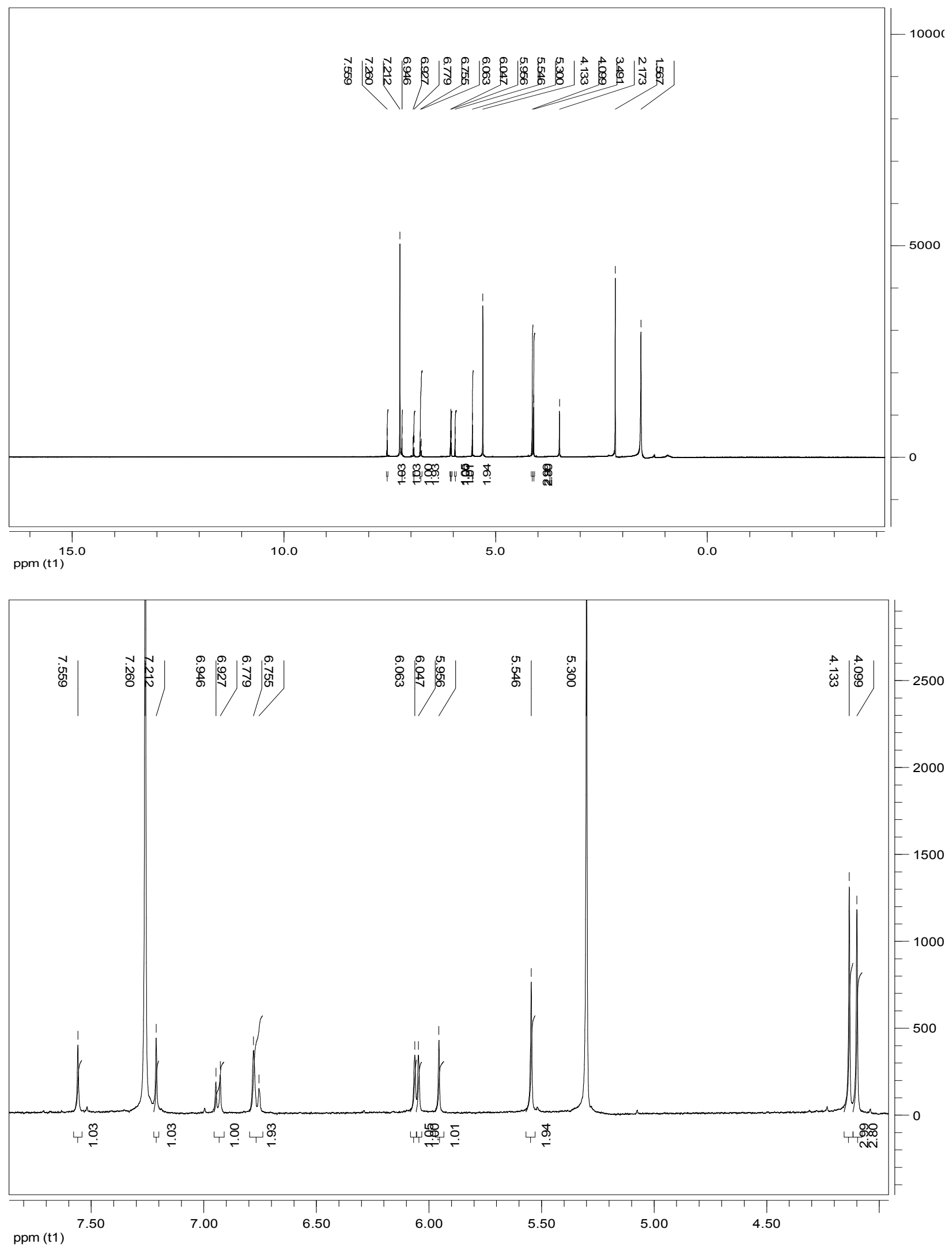

Figure S12a. ${ }^{1} \mathrm{H}$ NMR spectrum of phyllanthusmin A (6). 

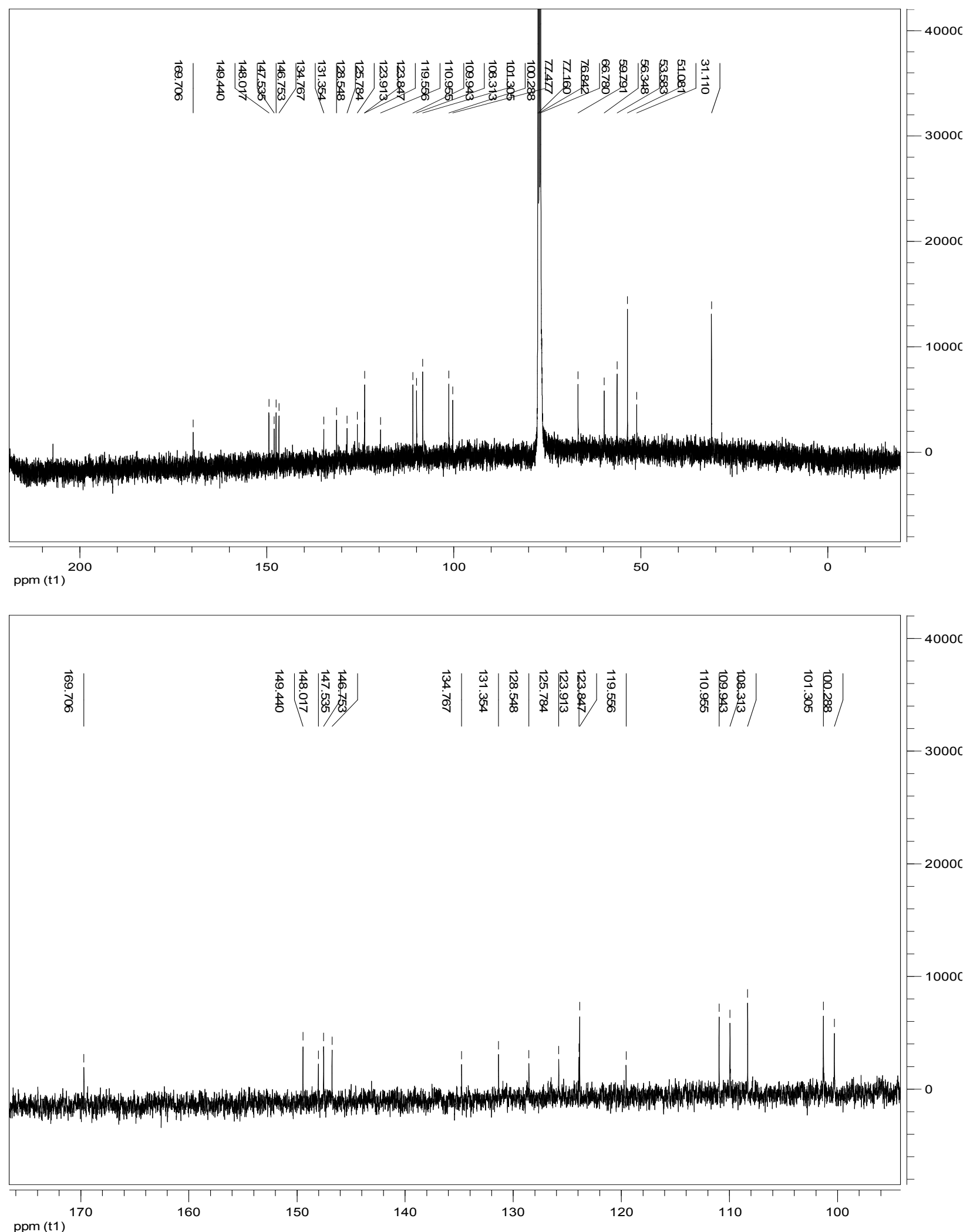


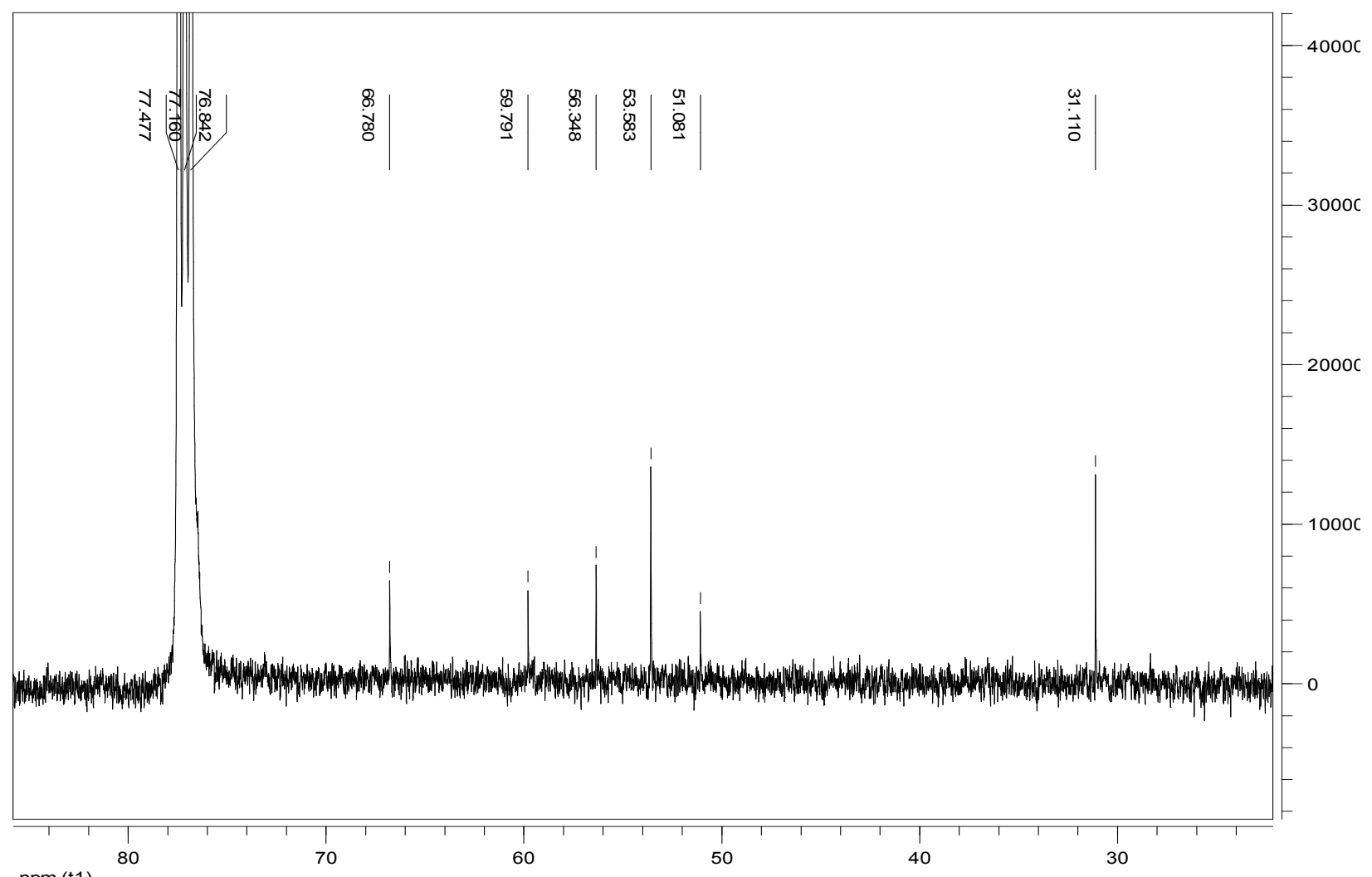

ppm (t1)

Figure S12b. C NMR spectrum of phyllanthusmin A (6).

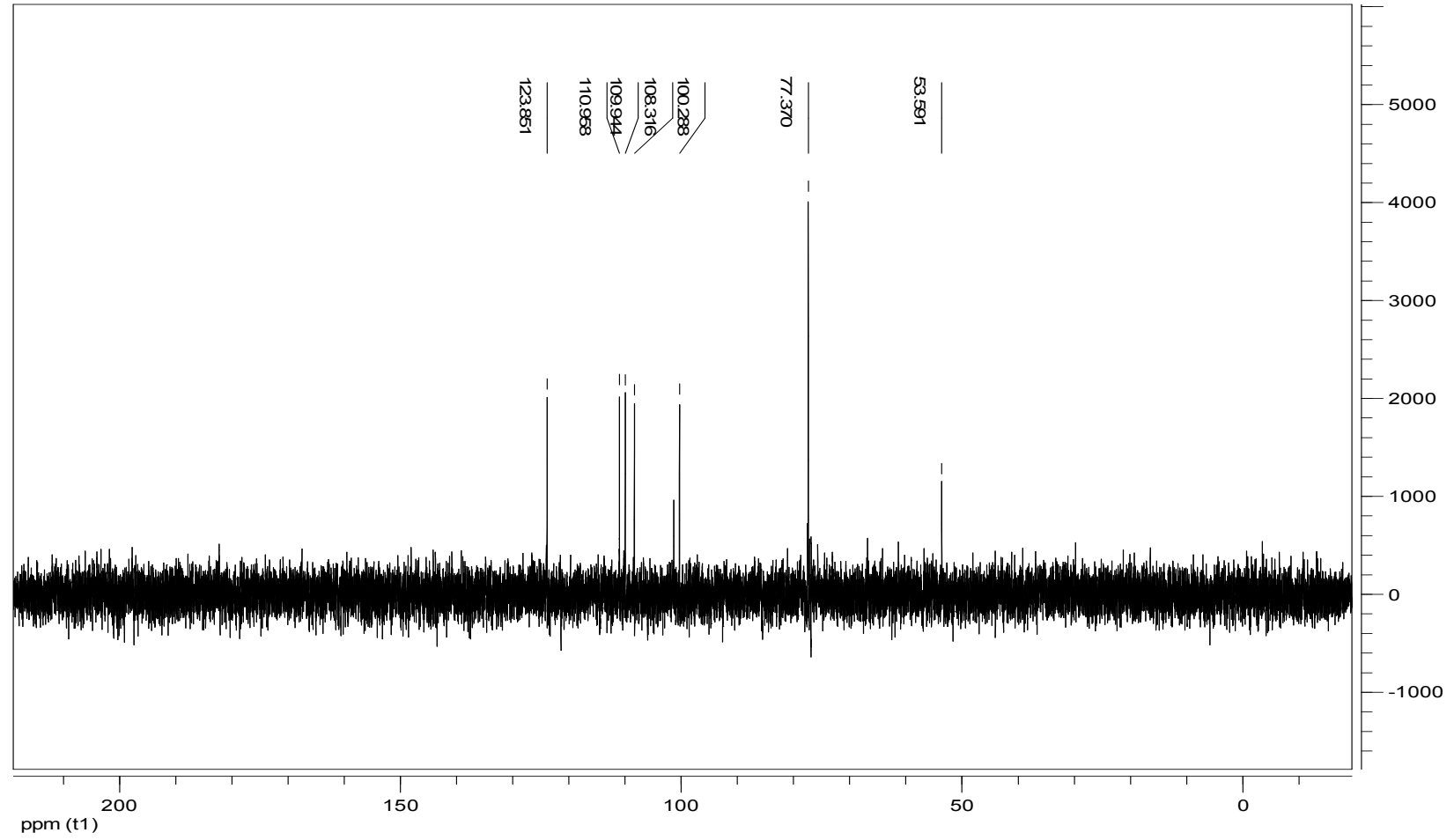

Figure S12c. DEPT 90 NMR spectrum of phyllanthusmin A (6). 


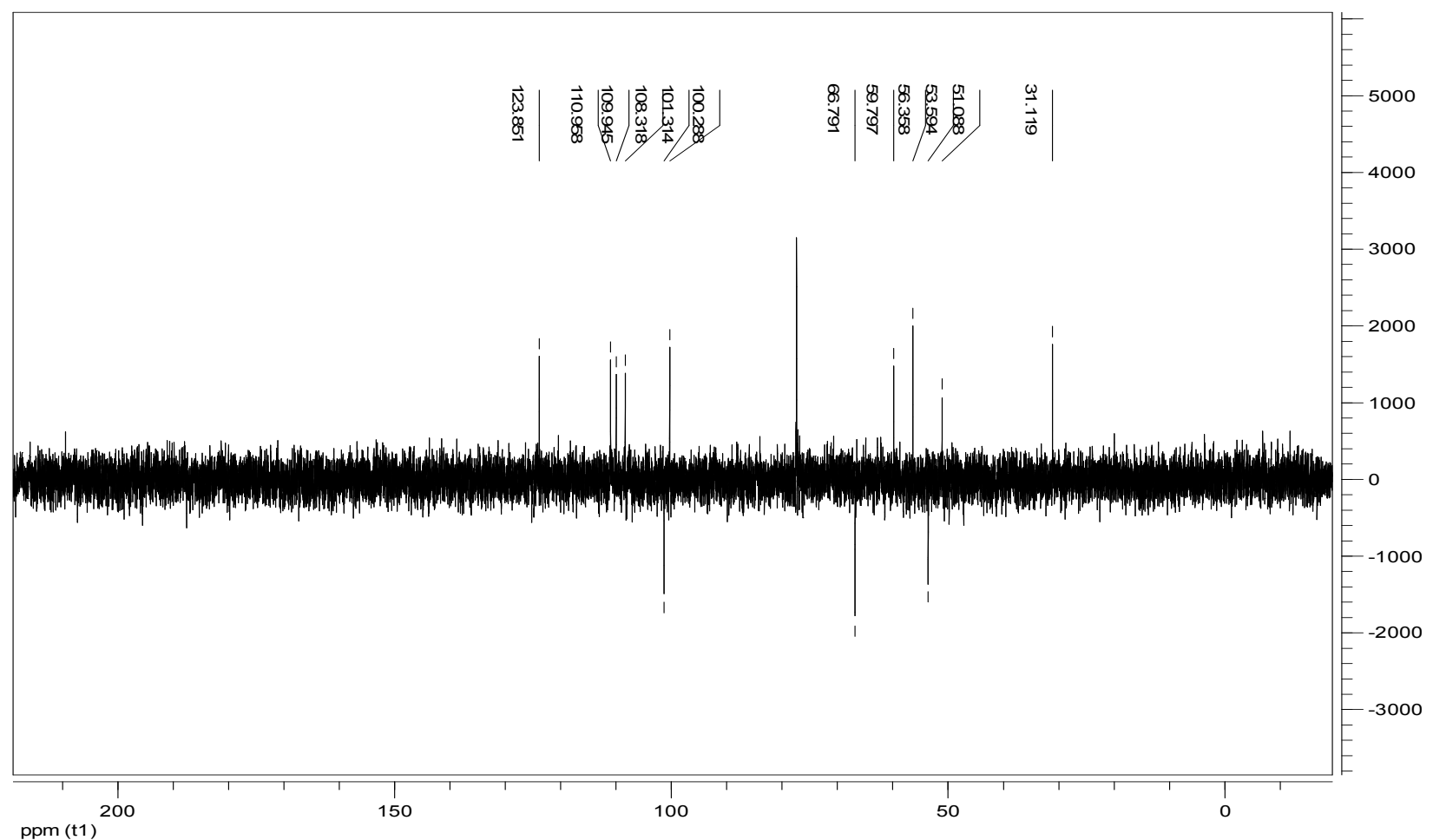

Figure S12d. DEPT 135 NMR spectrum of phyllanthusmin A (6).

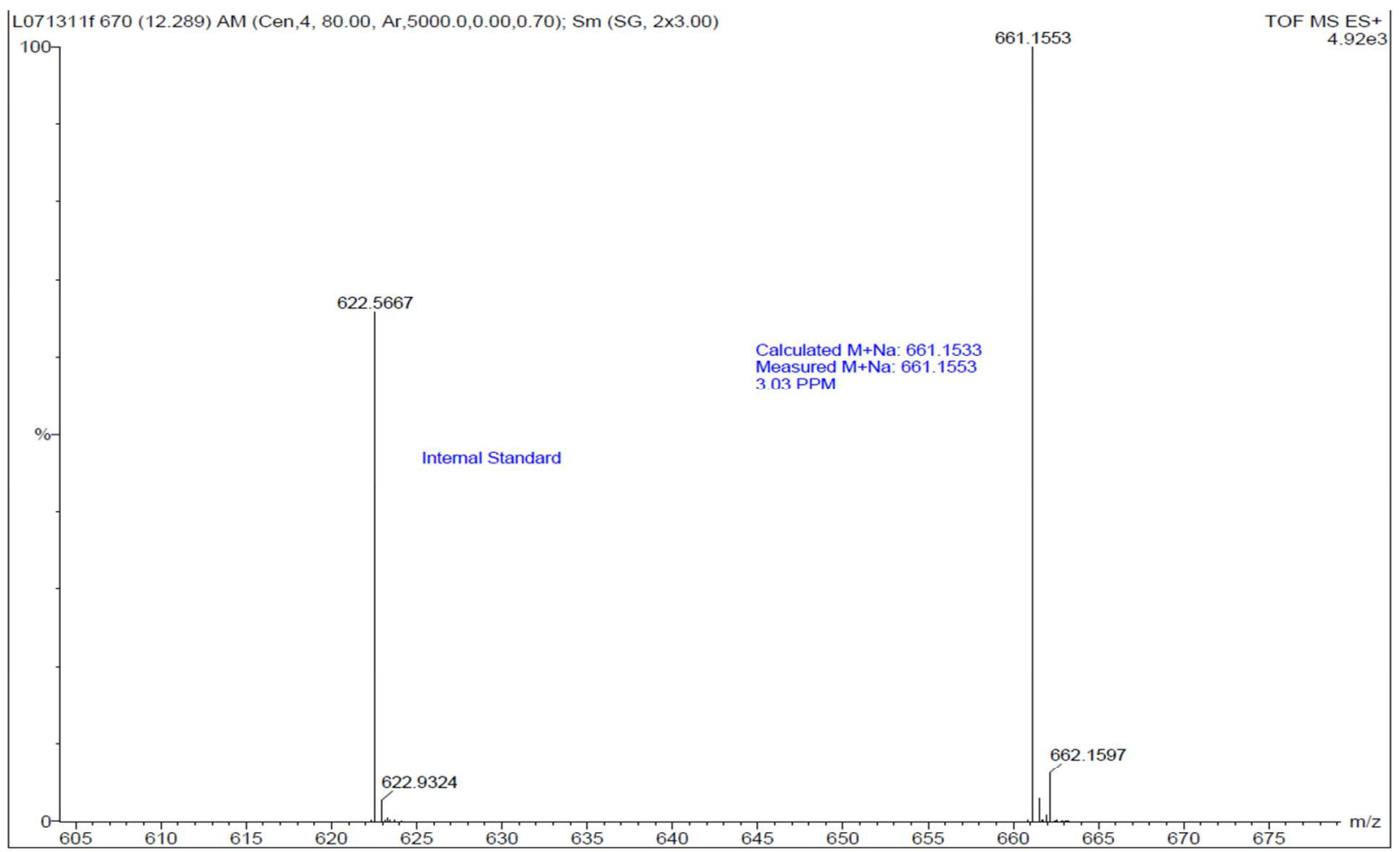

Figure S13. MS spectrum of 7-O-[(2,3,4-tri- $O$-acetyl)- $\alpha$-L-arabinopyranosyl)]diphyllin (7). 

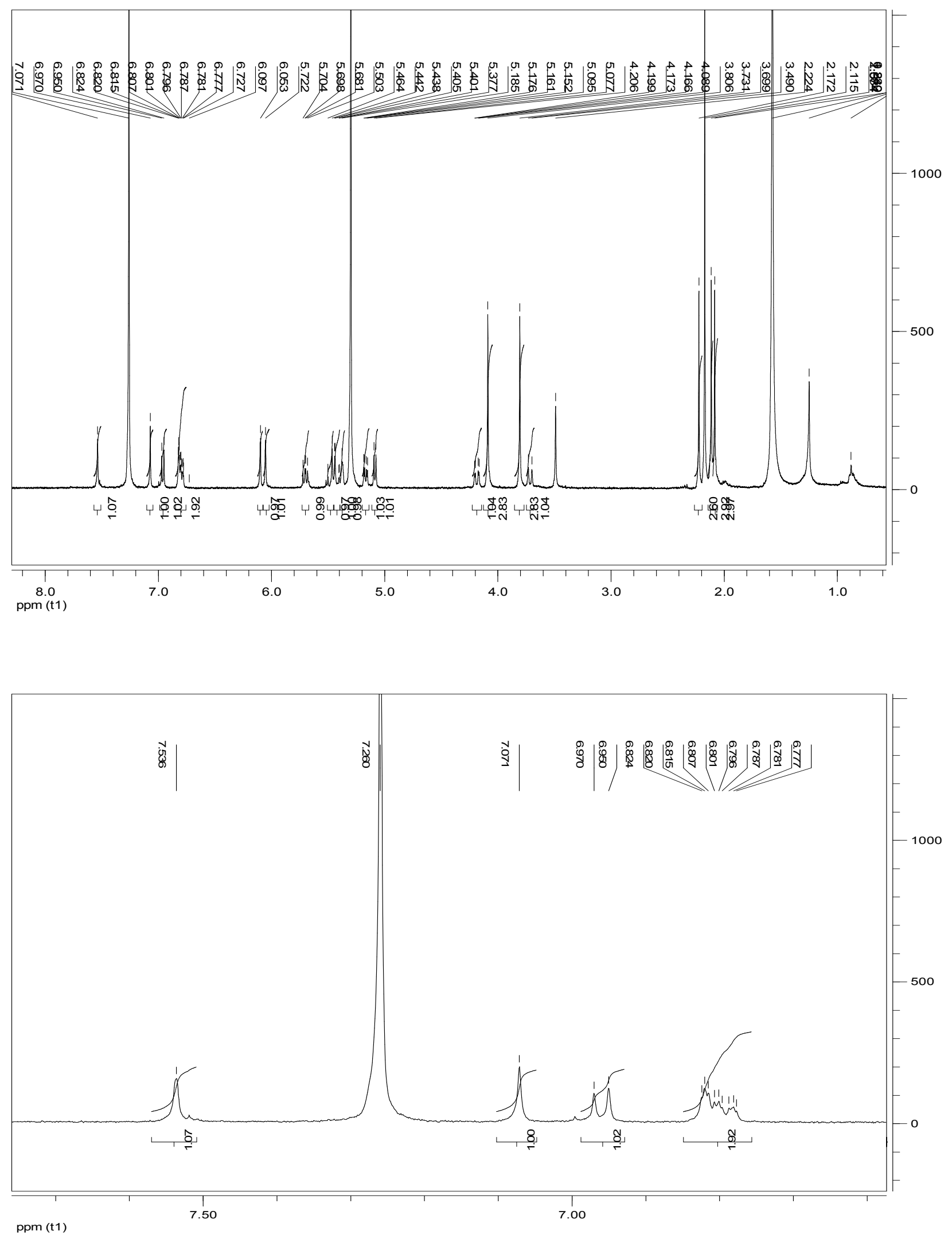


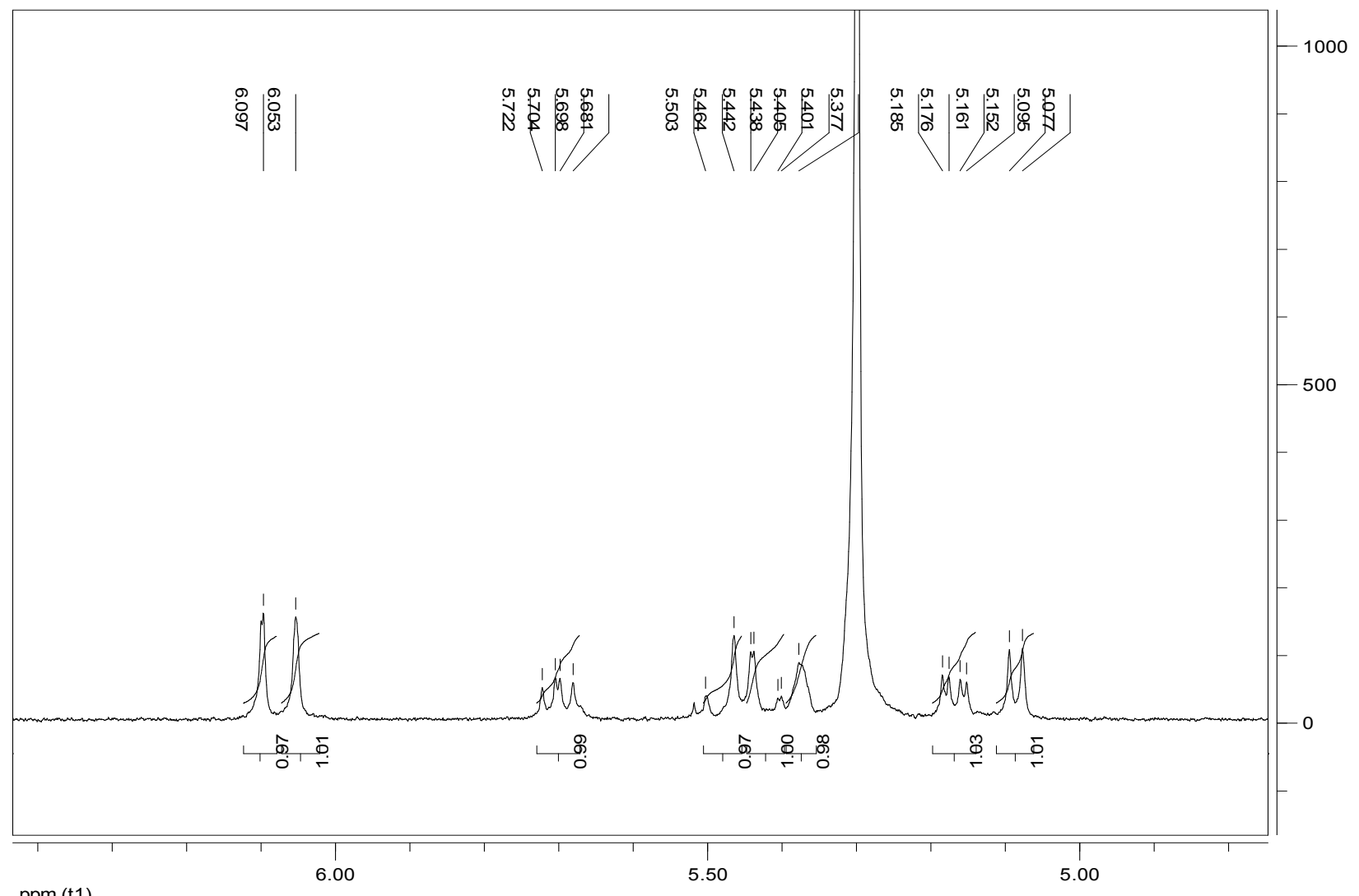

ppm (t1)

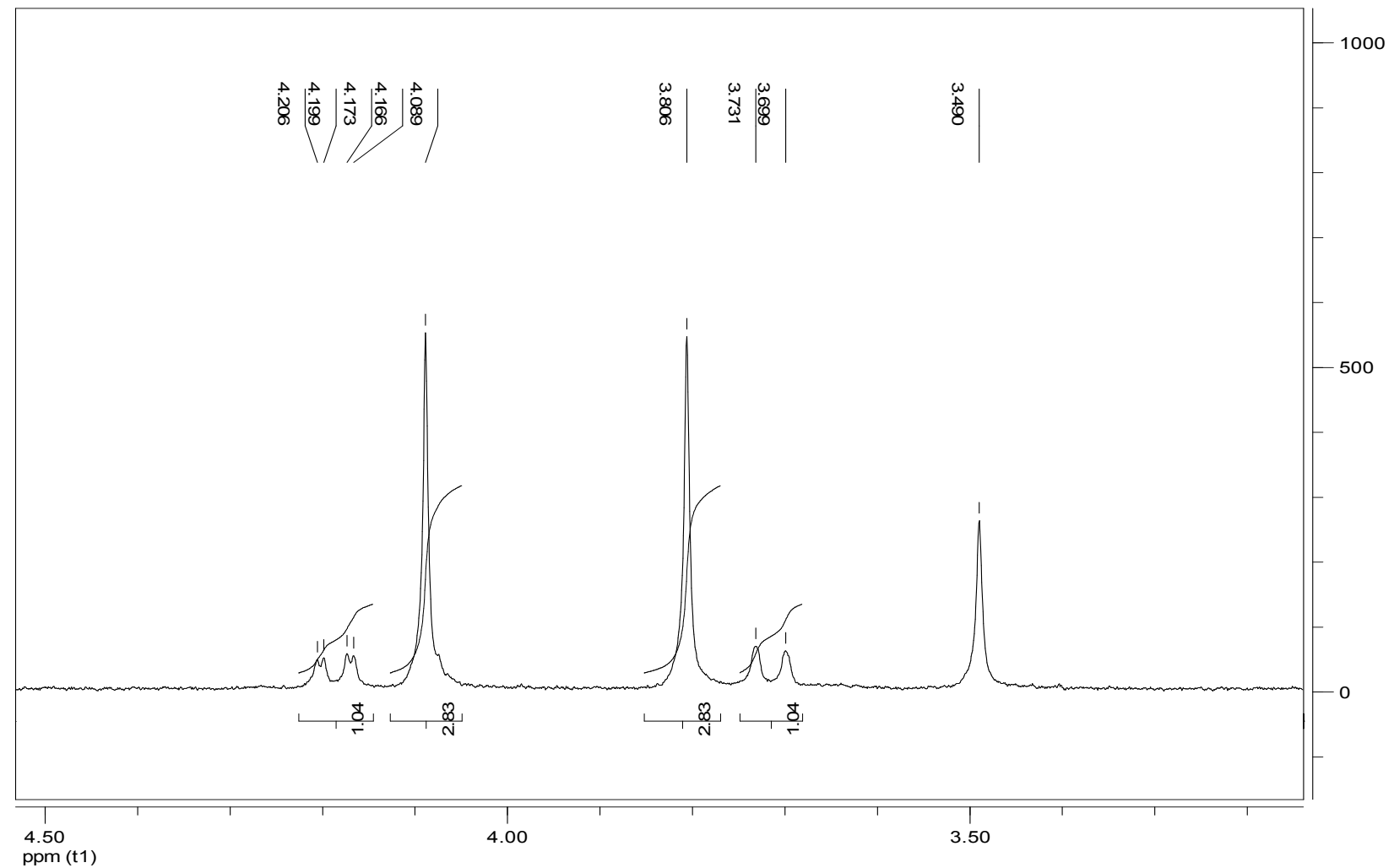




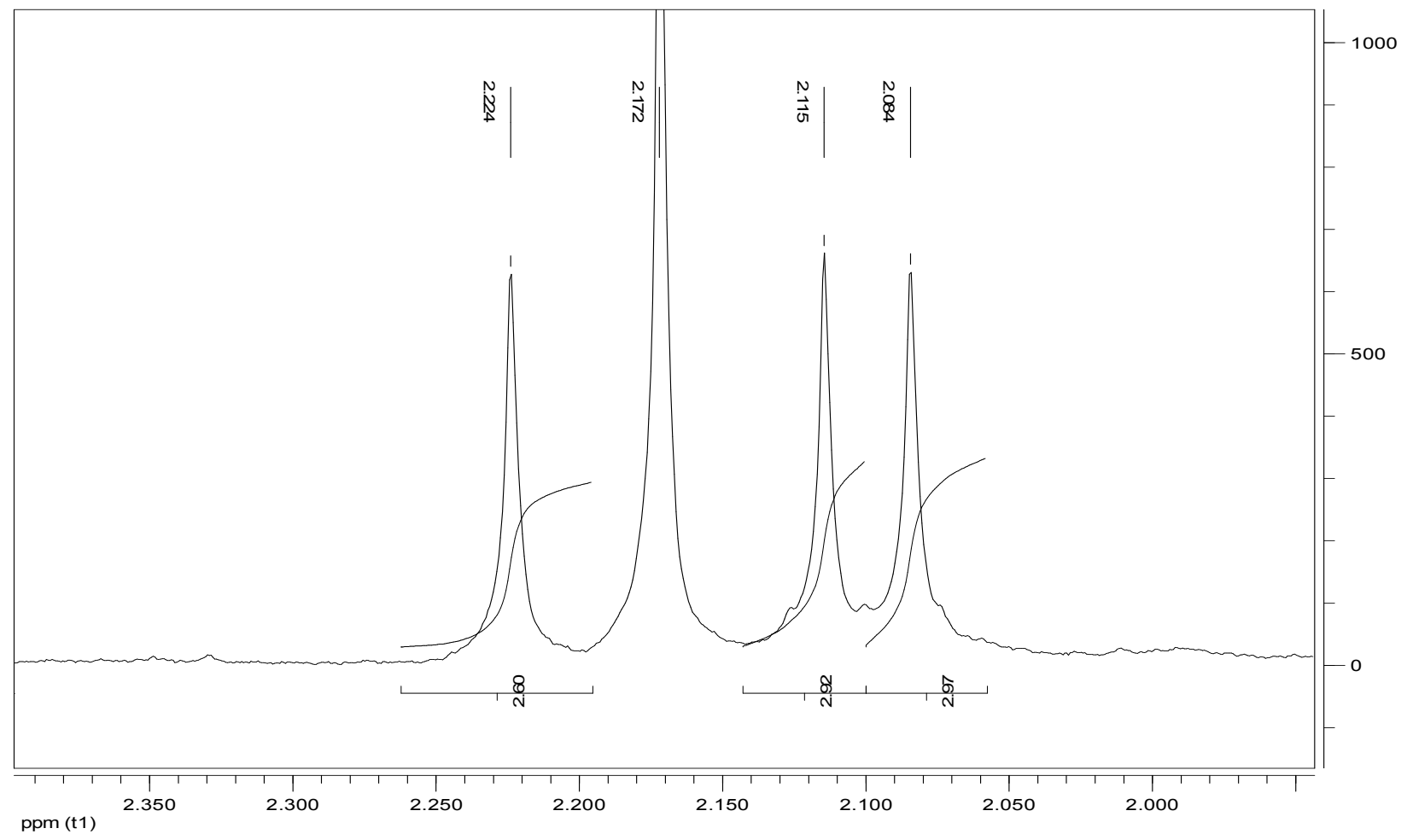

Figure S14a. ${ }^{1} \mathrm{H}-\mathrm{NMR}$ spectrum of 7-O-[(2,3,4-tri-O-acetyl)- $\alpha$-L-arabinopyranosyl)]diphyllin (7). 


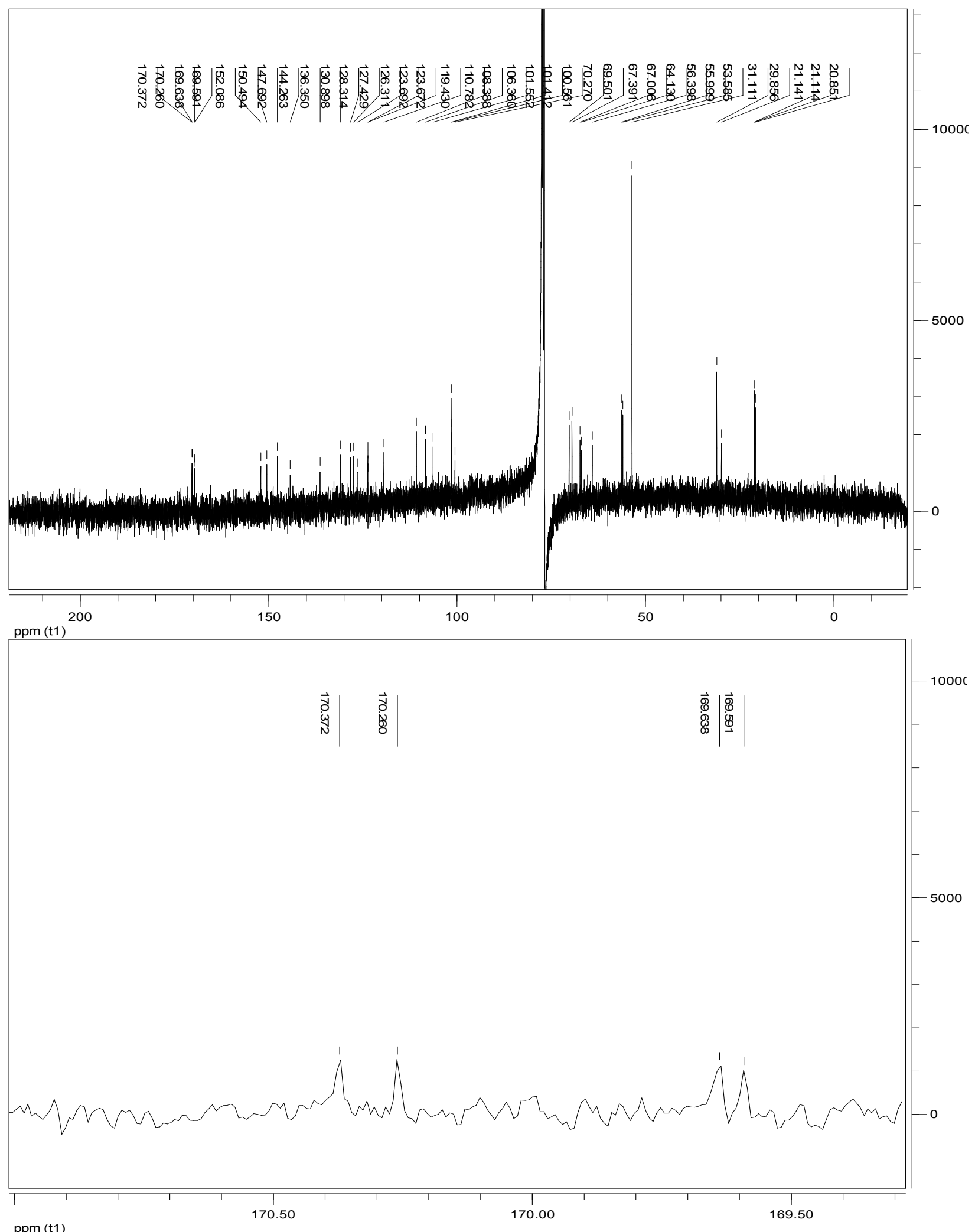

ppm (t1) 


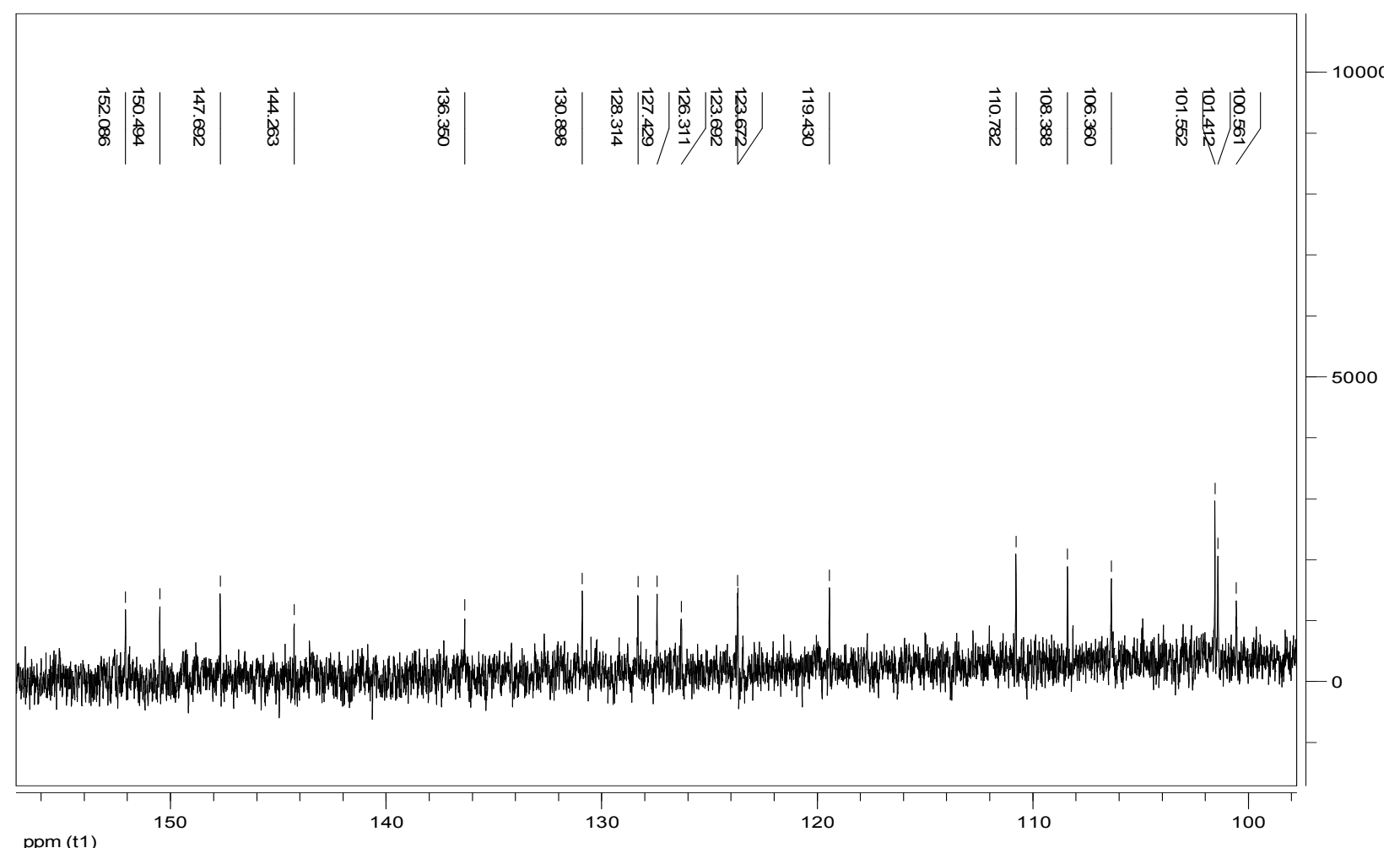
ppm (t1)

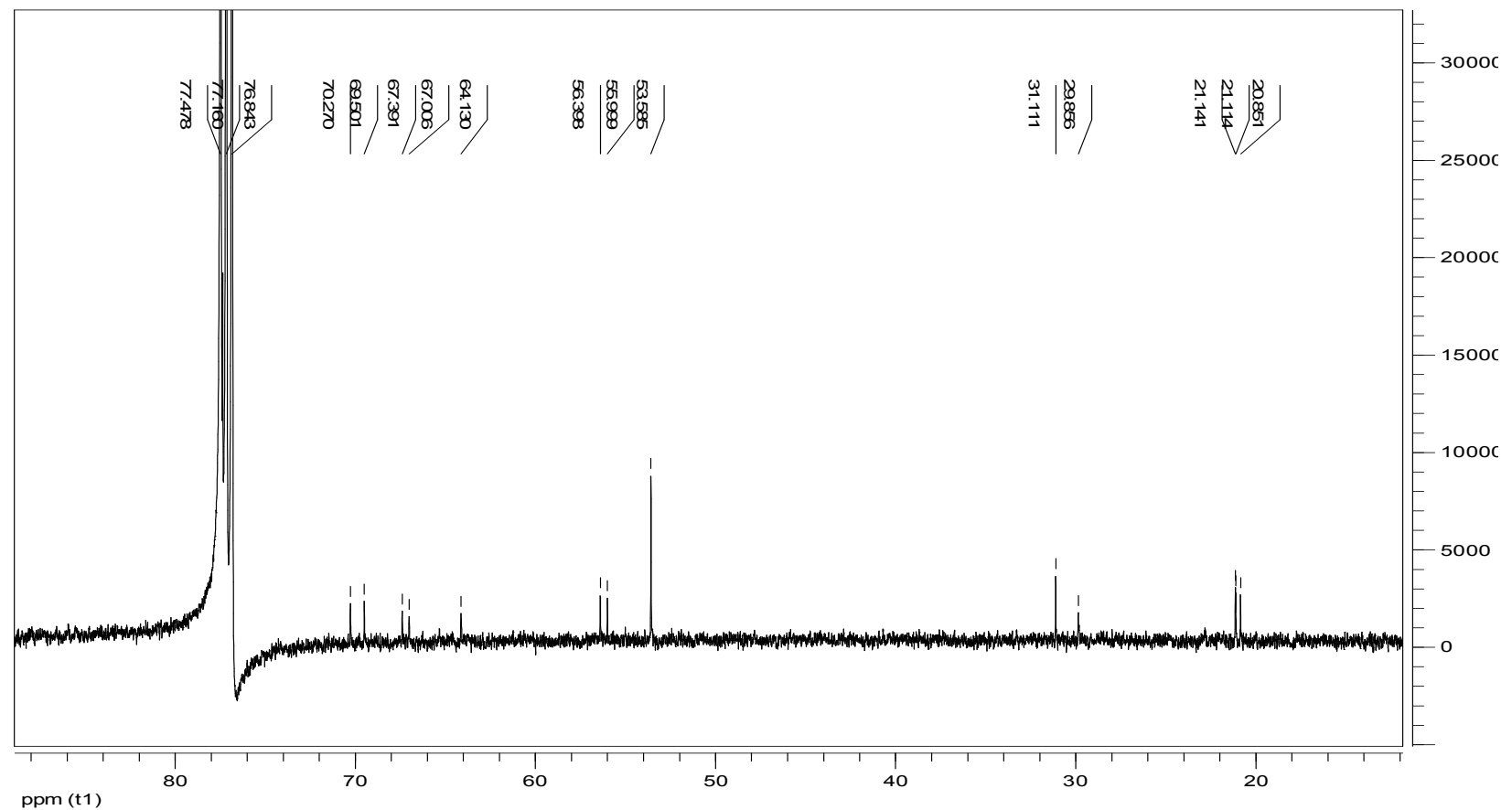

Figure S14b. ${ }^{13} \mathrm{C}-\mathrm{NMR}$ spectrum of 7-O-[(2,3,4-tri- $O$-acetyl)- $\alpha$-L-arabinopyranosyl $\left.)\right]$ diphyllin (7). 


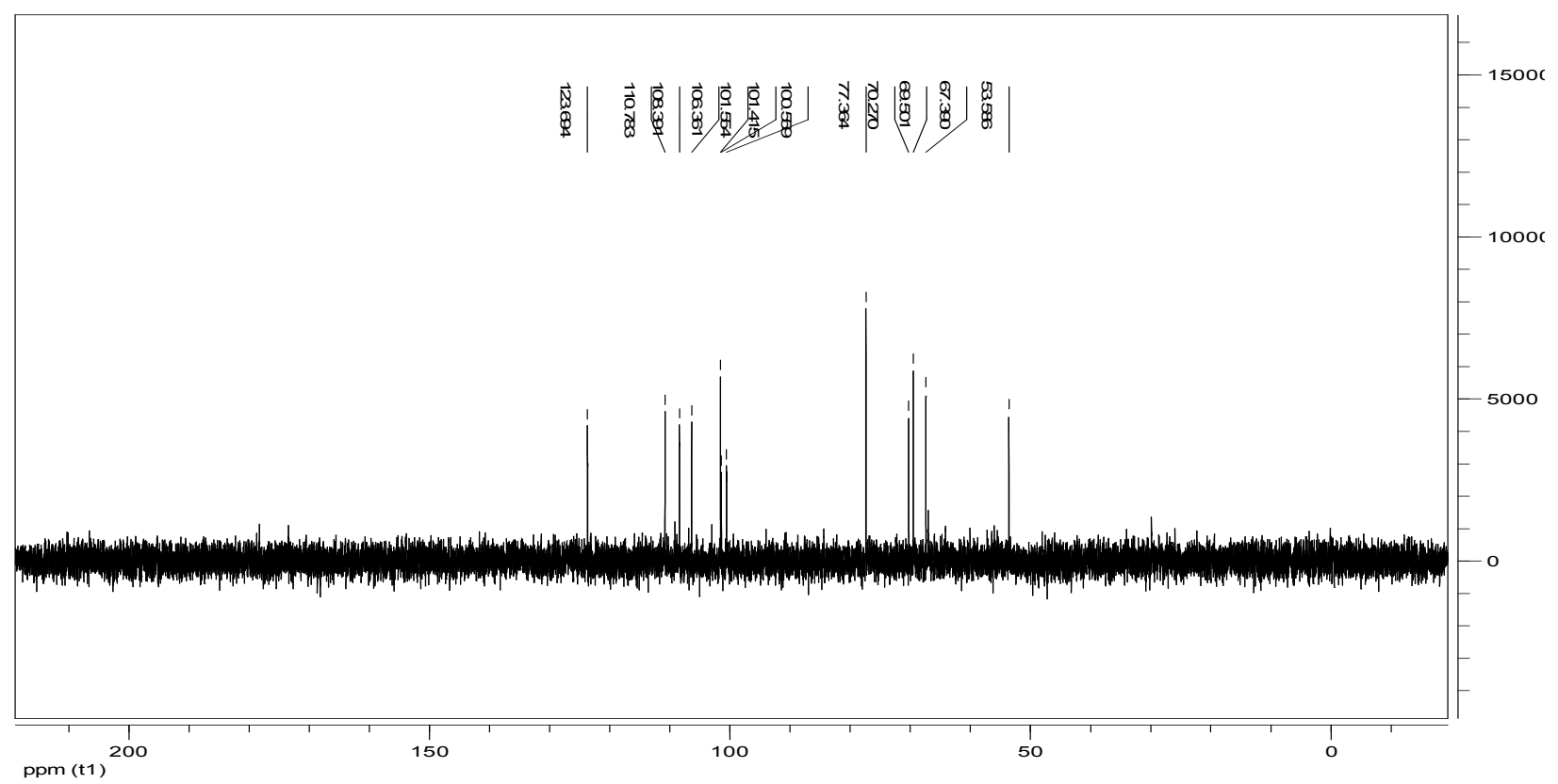

Figure S14c. DEPT90 NMR spectrum of 7-O-[(2,3,4-tri- $O$-acetyl $)-\alpha-\mathrm{L}-$ arabinopyranosyl)]diphyllin (7).

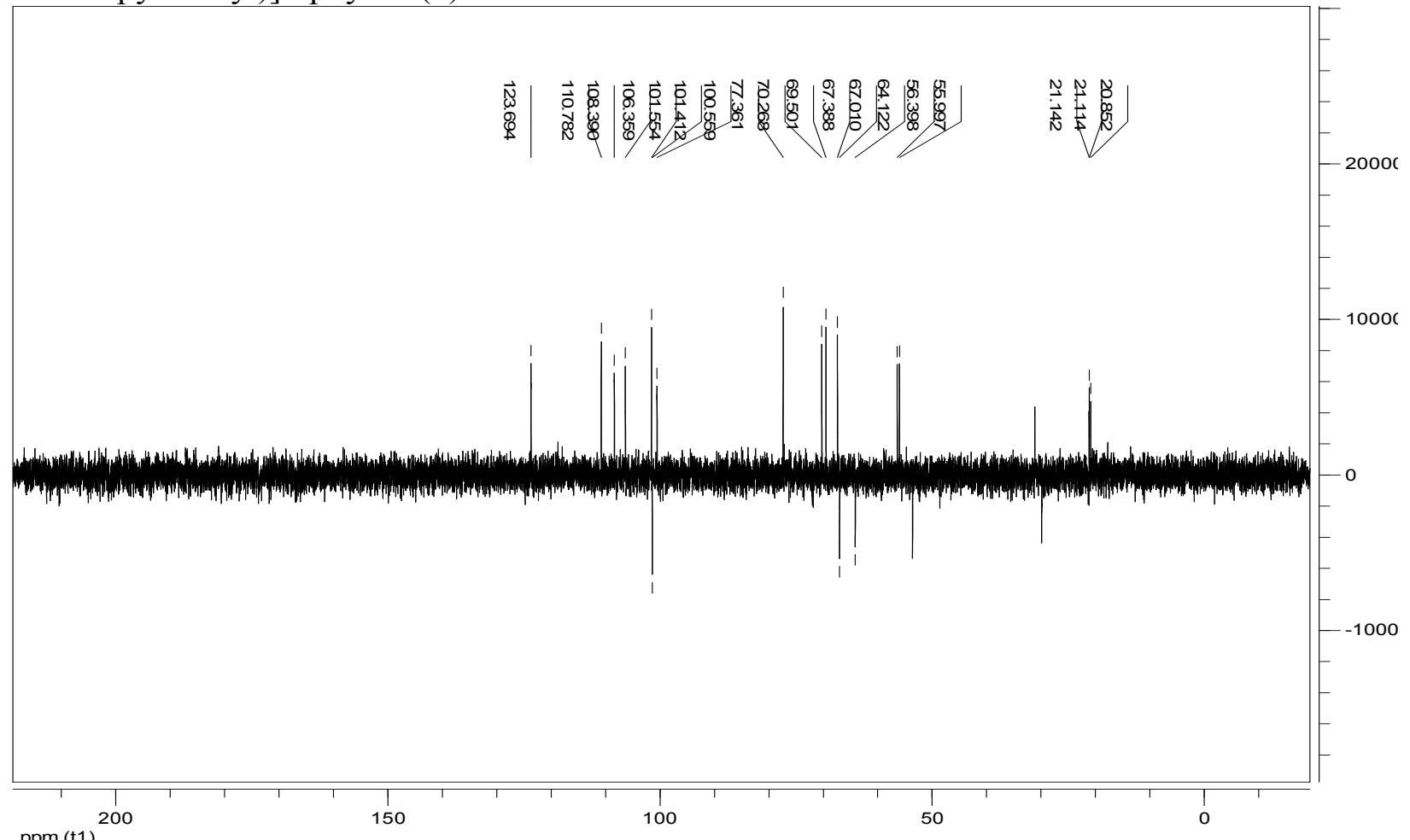

Figure S14d. DEPT135 NMR spectrum of 7-O-[(2,3,4-tri- $O$-acetyl)- $\alpha$-L-

arabinopyranosyl)]diphyllin (7). 


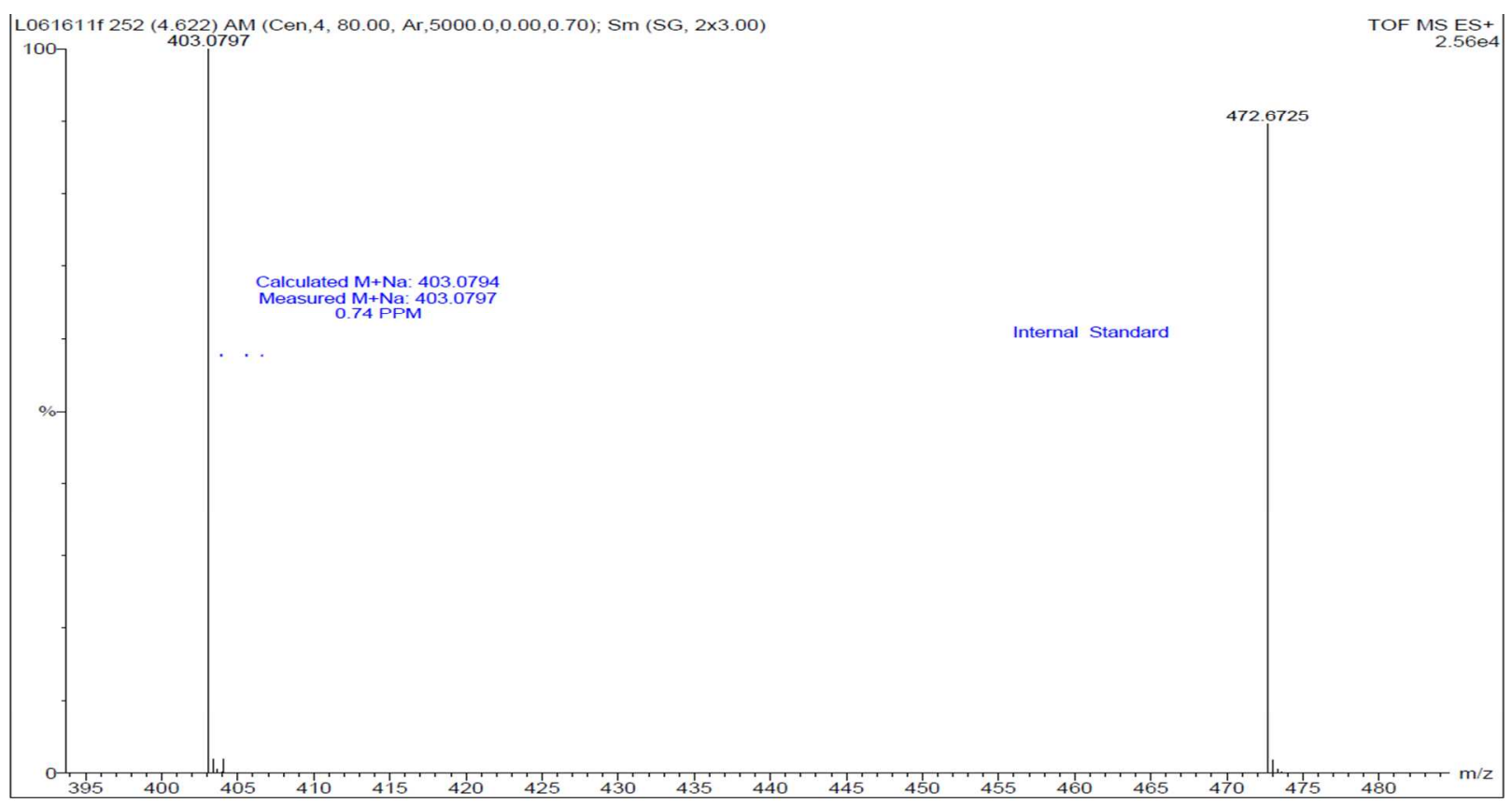

Figure S15. MS spectrum of diphyllin (8).

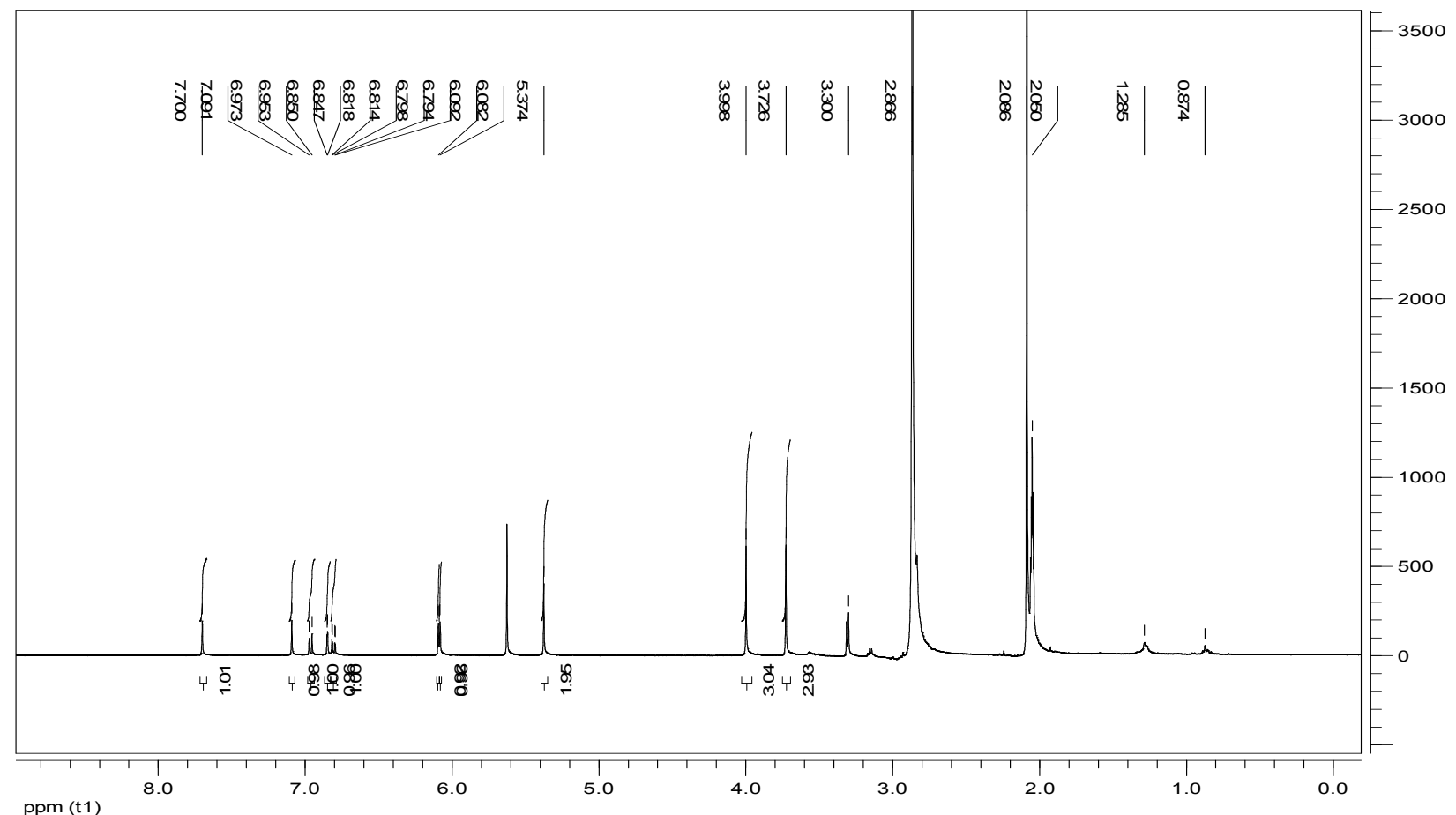




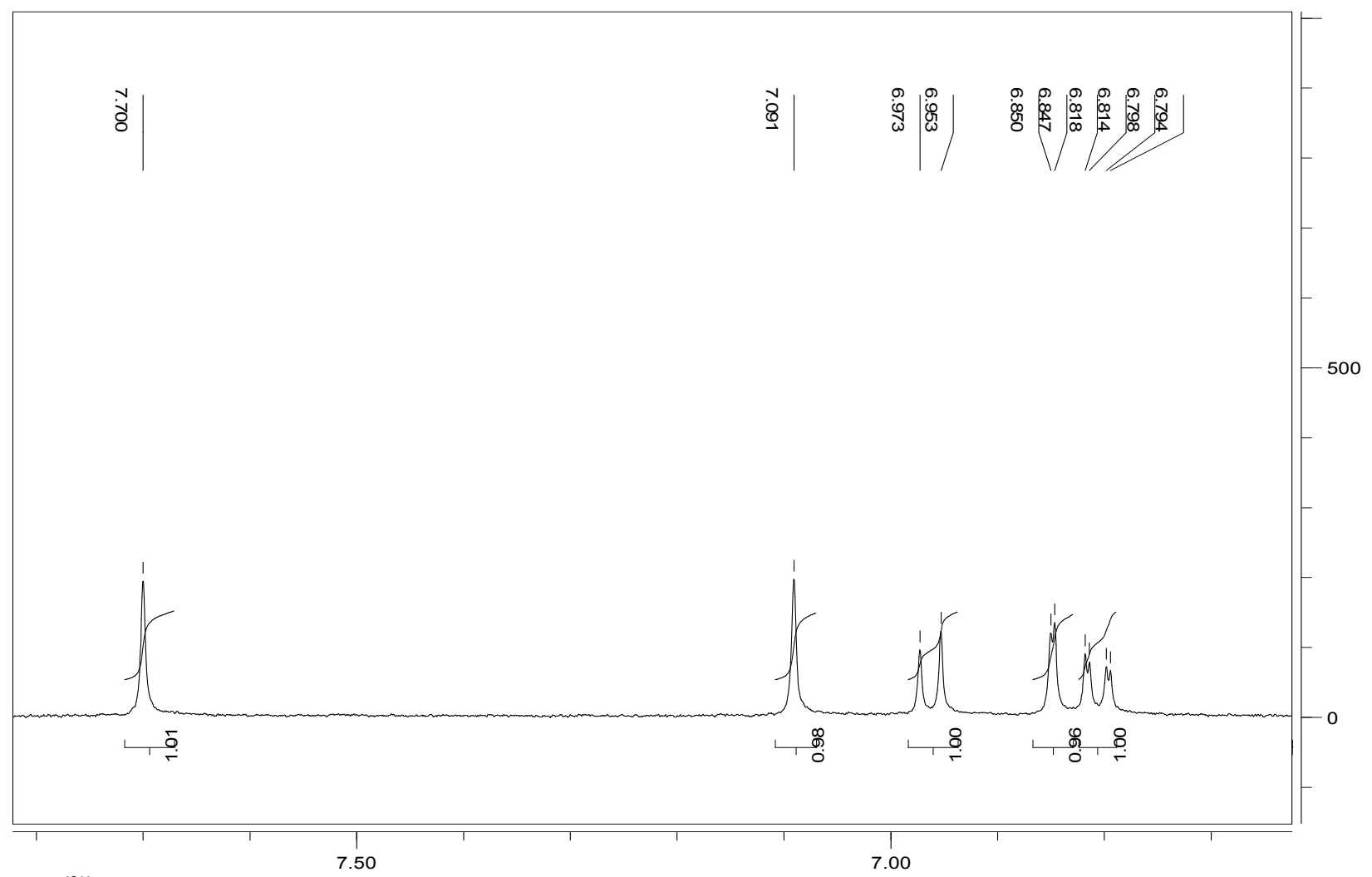

ppm (f1)

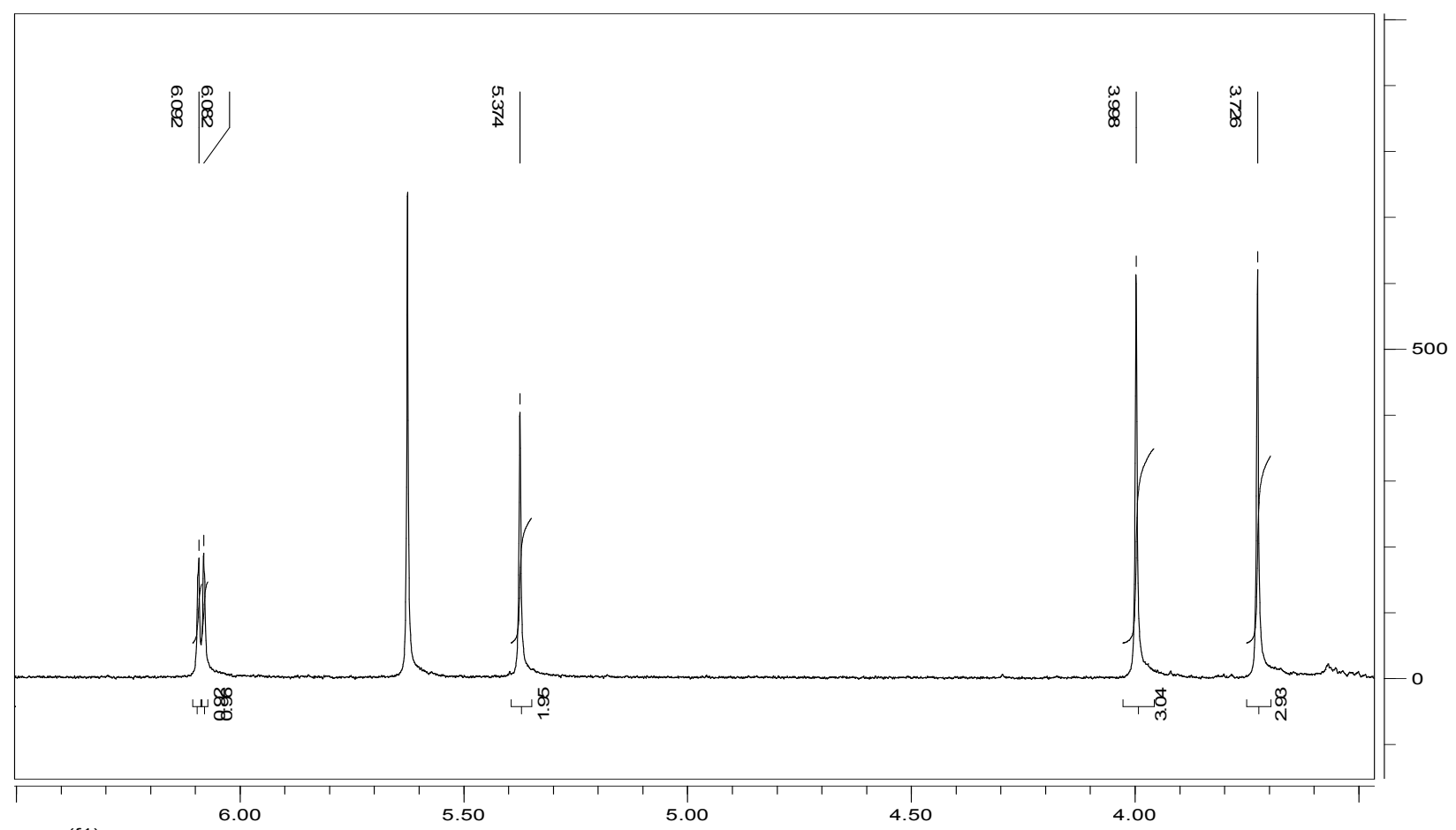

ppm (f1)

Figure S16a. ${ }^{1} \mathrm{H}$ NMR spectrum of diphyllin (8). 


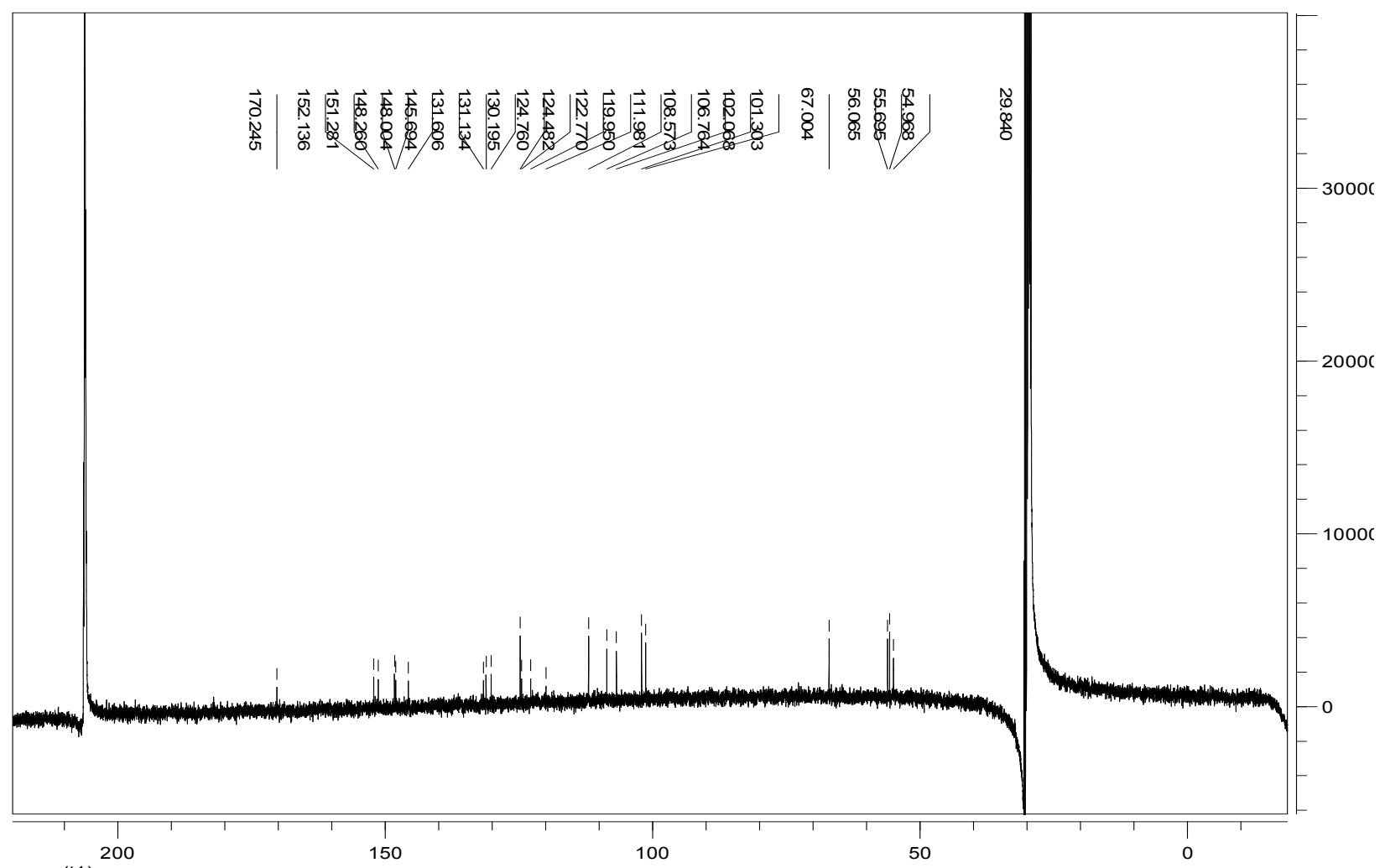

ppm (t1)

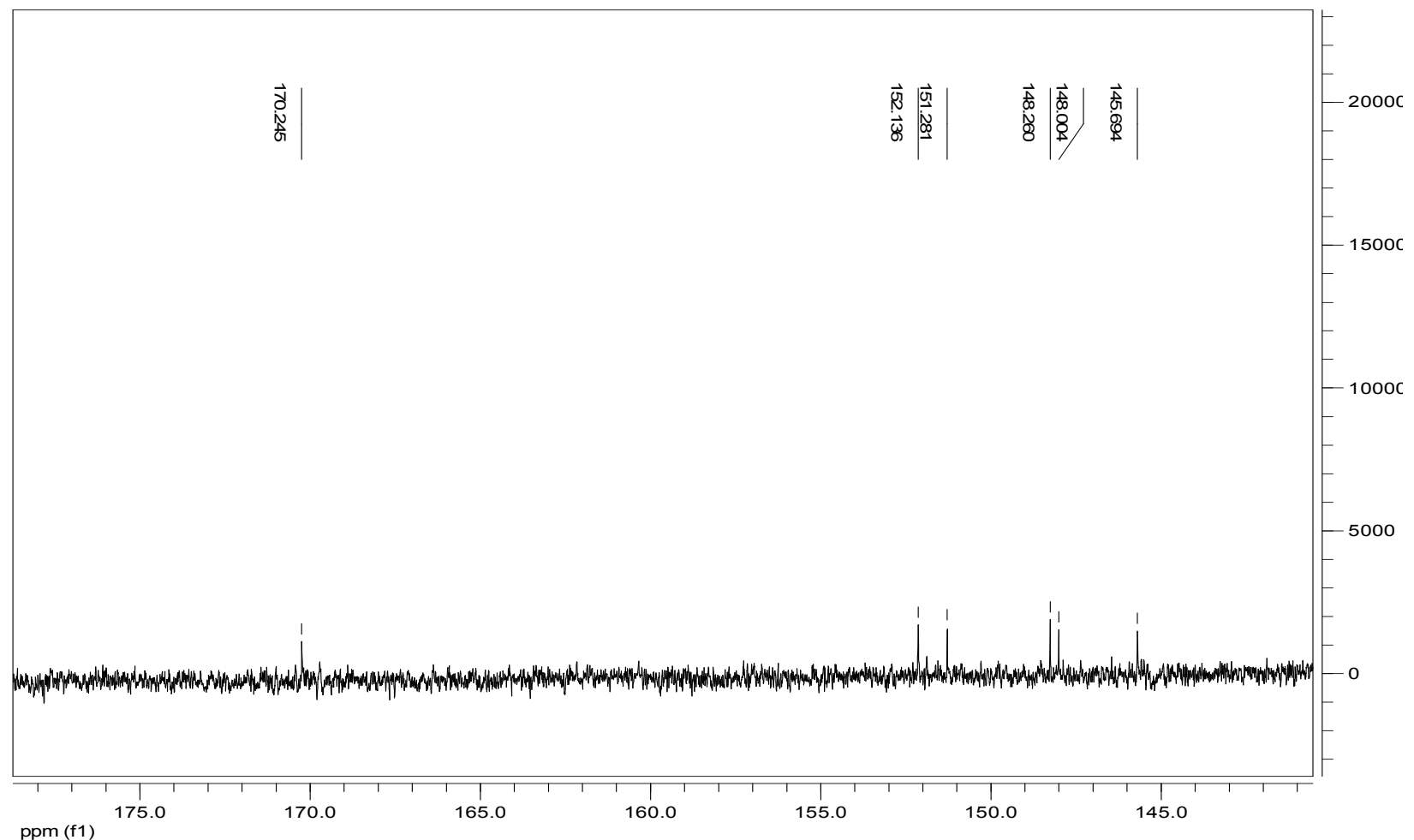




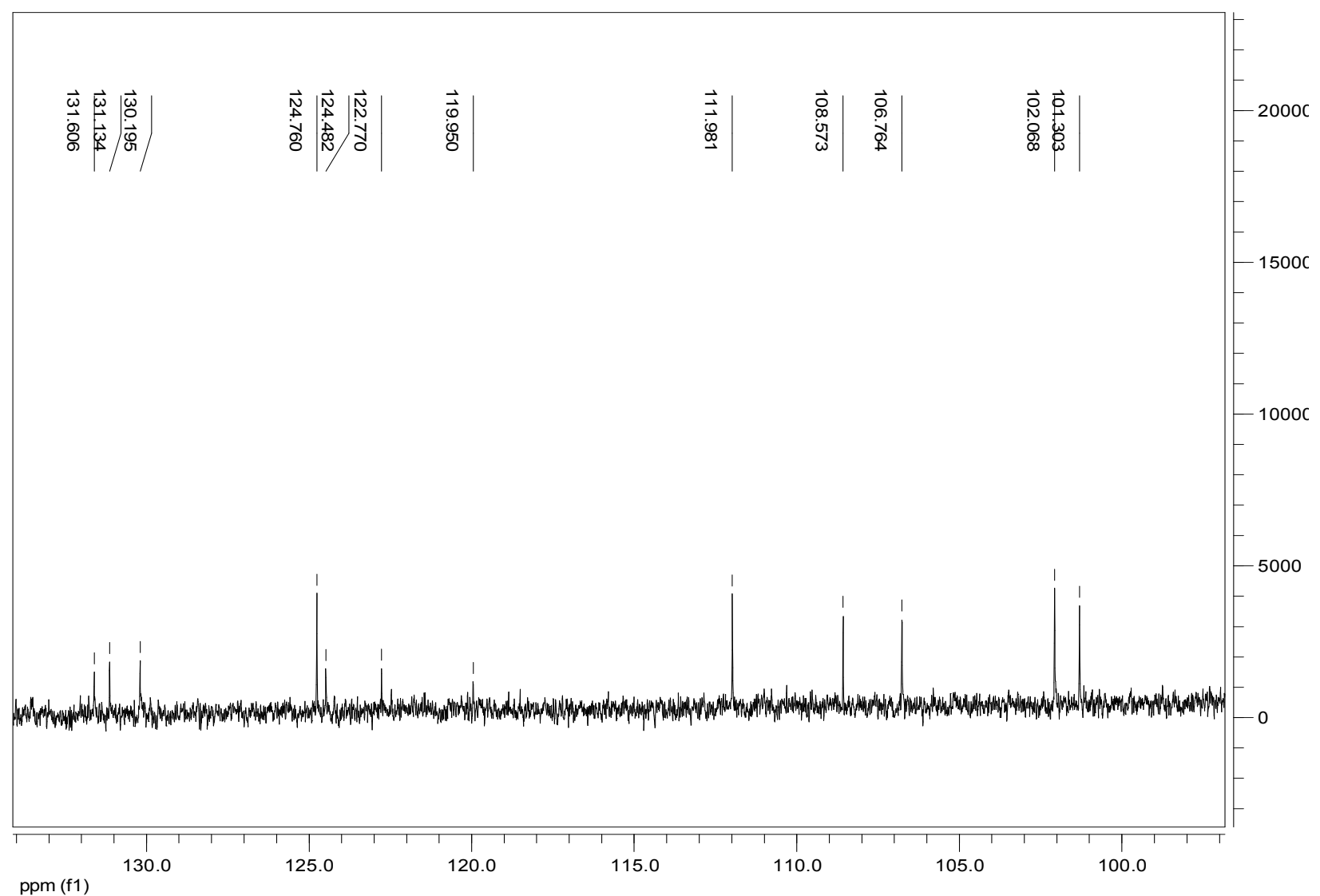

Figure S16b. ${ }^{13} \mathrm{C}$ NMR spectrum of diphyllin (8).

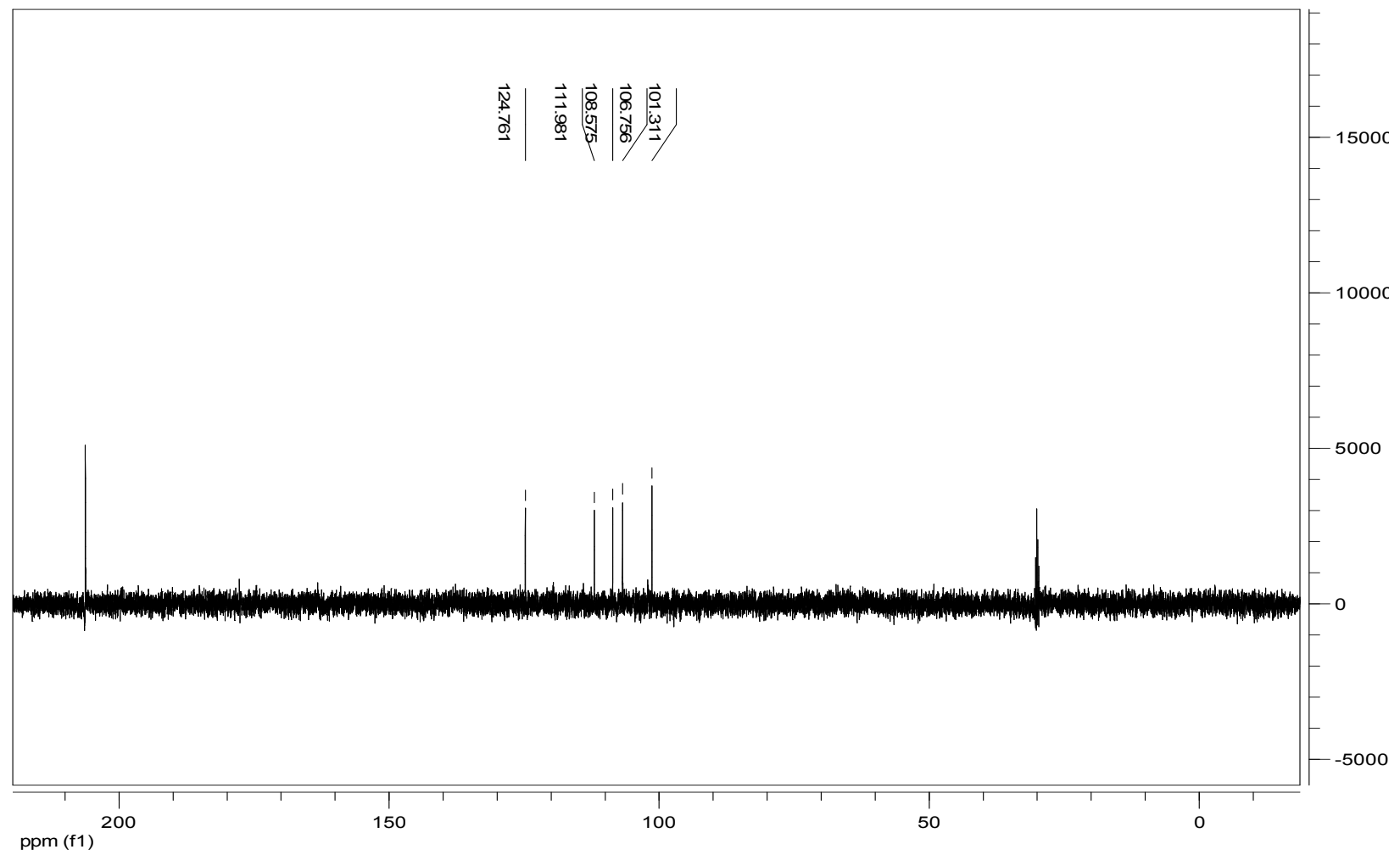


Figure S16c. DEPT 90 NMR spectrum of diphyllin (8).

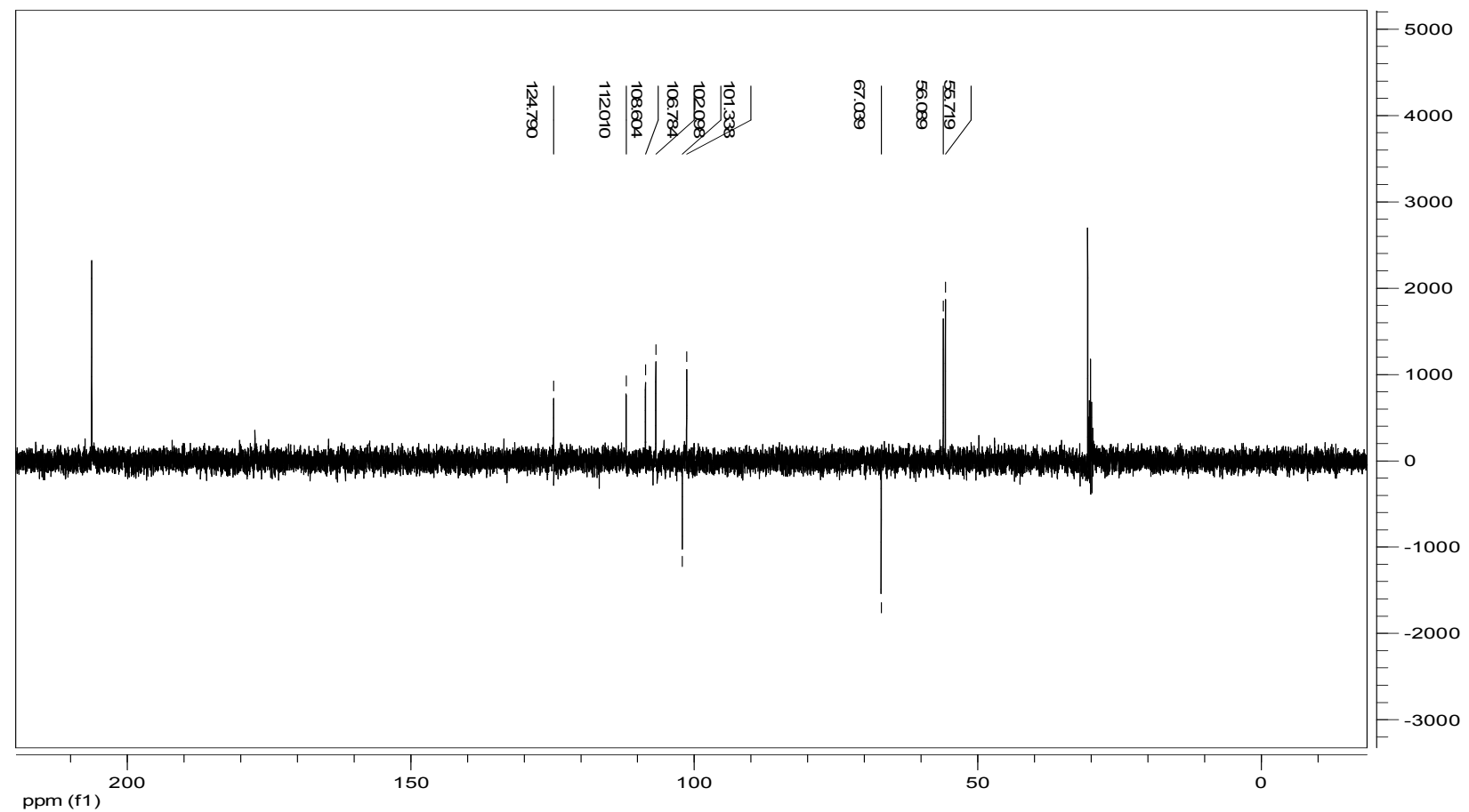

Figure S16d. DEPT 135 NMR spectrum of diphyllin (8).

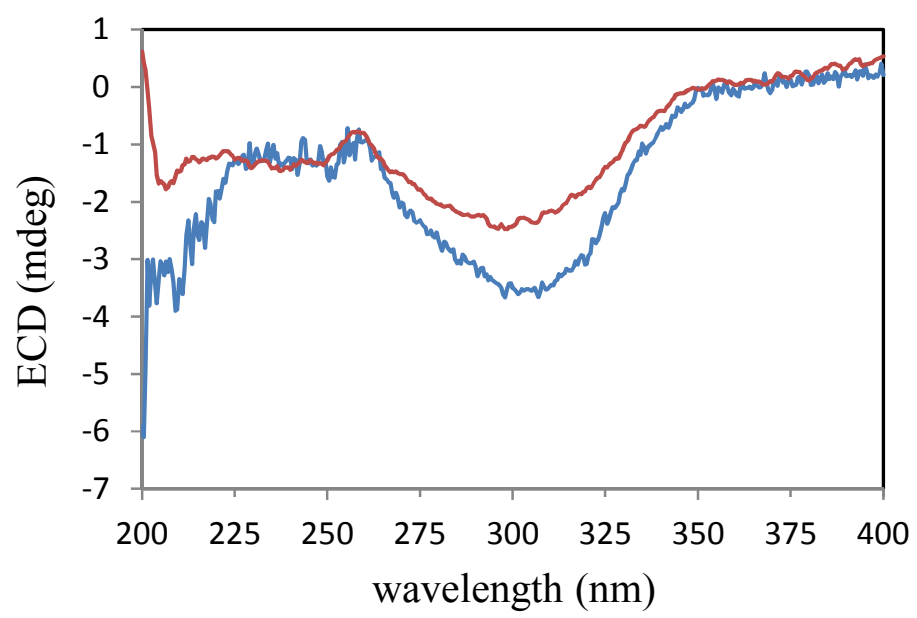

Figure S17. ECD spectra of phyllanthusmins D (1, blue) and E (2, red). The data were obtained in $\mathrm{MeOH}$ corrected by subtracting a spectrum of the appropriate solution in the absence of the samples recorded under identical conditions. 


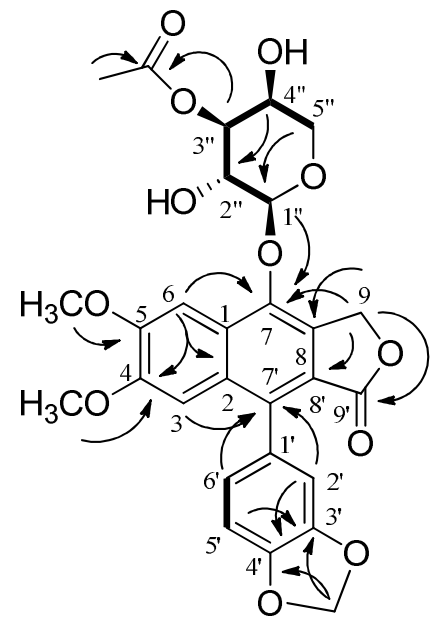

phyllanthusmin E (2)

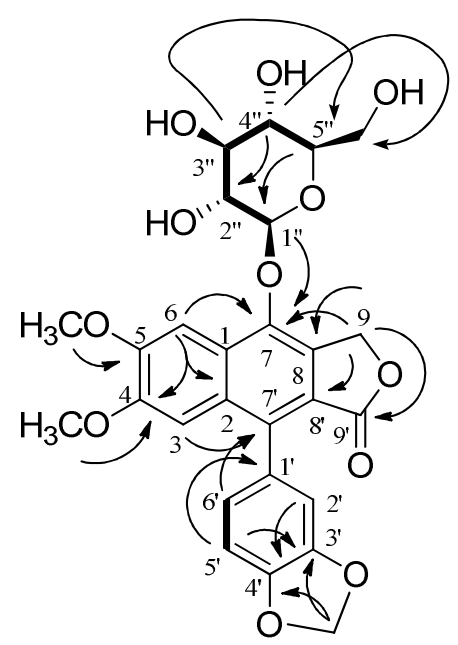

cleistanthin B (5)

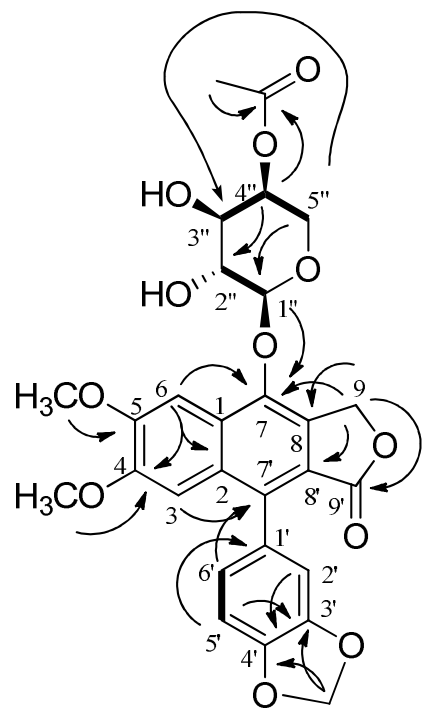

phyllanthusmin B (3)

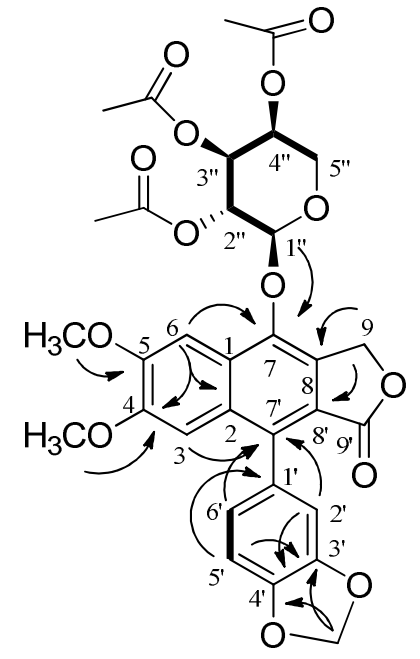

7-O-[(2,3,4-tri- $O$-acetyl)- $\alpha-\mathrm{L}-$ arabinopyranosyl)]diphyllin (7)

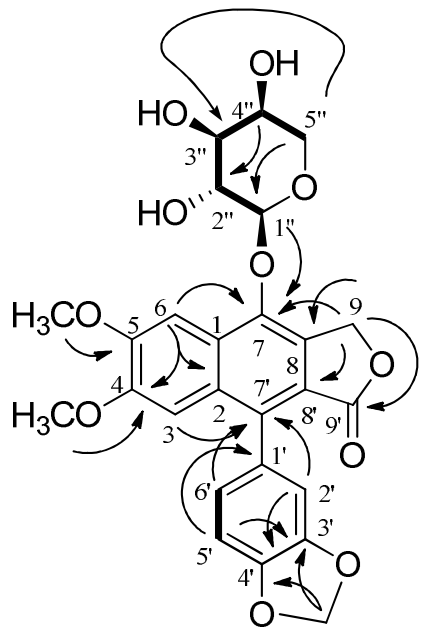

phyllanthusmin C (4)

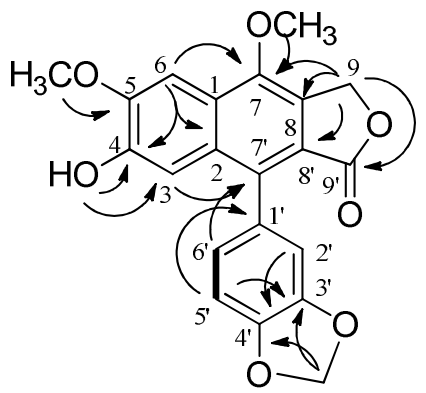

phyllanthusmin A (6)

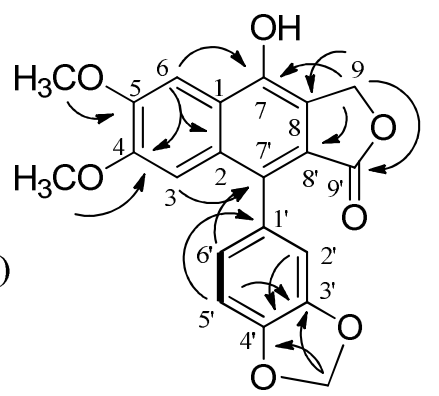

diphyllin (8)

Figure S18. COSY ( - ) and the key HMBC ( $)$ NMR correlations of compounds $\mathbf{2}-\mathbf{8}$. 


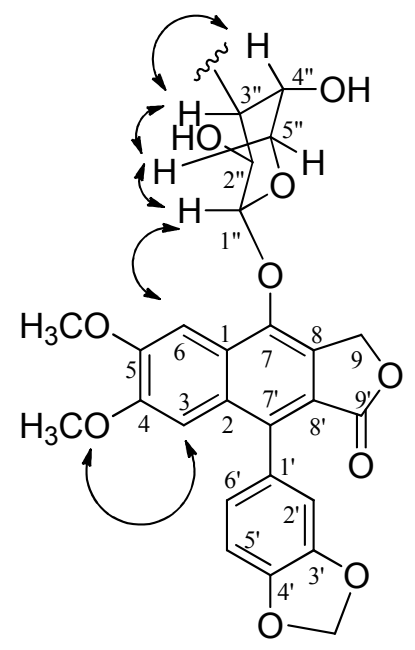

phyllanthusmin E (2)

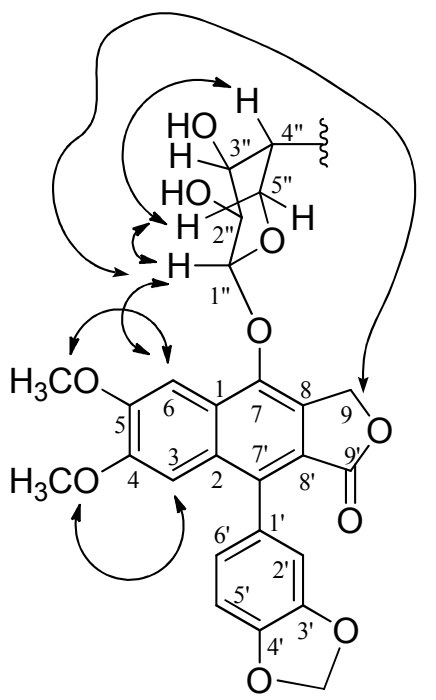

phyllanthusmin B (3)

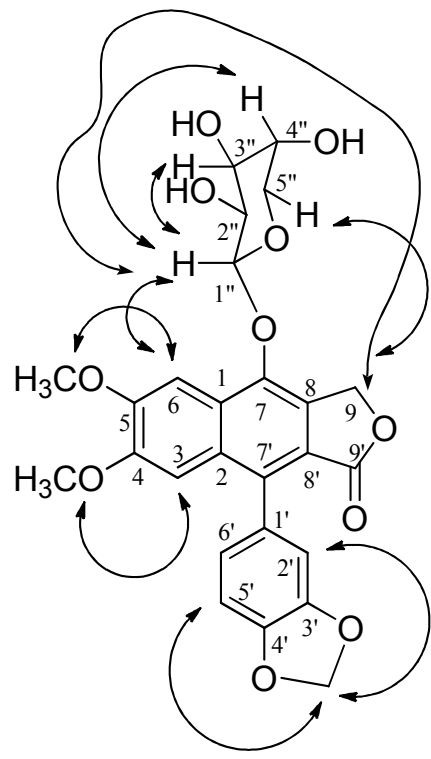

phyllanthusmin C (4)

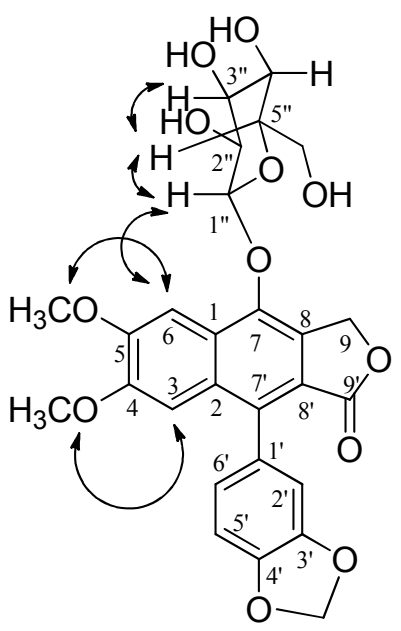

cleistanthin B (5)

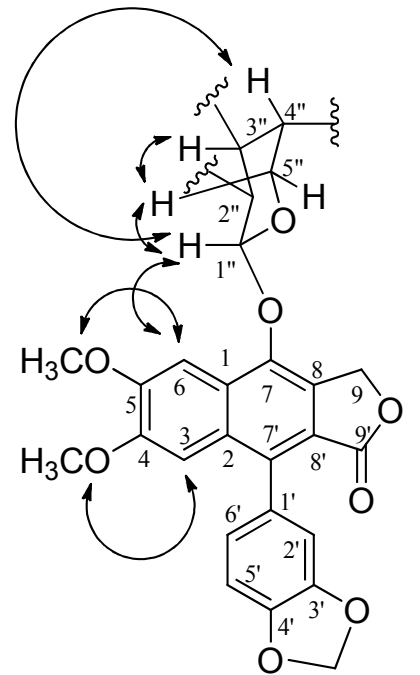

7-O-[(2,3,4-tri- $O$-acetyl $)-\alpha-\mathrm{L}-$ arabinopyranosyl)]diphyllin (7)

Figure S19. Selected NOESY $\left(\curvearrowleft,{ }^{1} \mathrm{H} \rightarrow{ }^{1} \mathrm{H}\right)$ correlations of compounds $\mathbf{2}-\mathbf{5}$ and 7. 


\section{Table S1. ${ }^{1}$ H NMR Spectroscopic Data of Compounds $3-5^{a}$}

\begin{tabular}{|c|c|c|c|}
\hline position & $3^{b}$ & $4^{c}$ & $5^{d}$ \\
\hline 3 & $7.00 \mathrm{~s}$ & 6.98 br s & $7.11 \mathrm{br} \mathrm{s}$ \\
\hline 6 & $8.17 \mathrm{~s}$ & $8.18 \mathrm{br} \mathrm{s}$ & $8.28 \mathrm{~s}$ \\
\hline \multirow[t]{2}{*}{9} & $5.40 \mathrm{~d}\left(8.4^{e}\right)$ & $5.47 \mathrm{~d}(15.2)$ & $5.45 \mathrm{dd}\left(15.0,3.0^{e}\right)$ \\
\hline & $5.51 \mathrm{~d}\left(7.8^{e}\right)$ & $5.55 \mathrm{~d}(15.2)$ & $5.77 \mathrm{dd}\left(15.0,3.0^{e}\right)$ \\
\hline $2^{\prime}$ & $6.94 \mathrm{~s}$ & $6.93 \mathrm{brs}$ & $6.90 \mathrm{dd}\left(15.6^{\mathrm{e}}, 1.2\right)$ \\
\hline $5^{\prime}$ & $7.06 \mathrm{~d}\left(12.0^{e}\right)$ & $7.05 \mathrm{~d}(8.0)$ & $6.99 \mathrm{dd}(7.8,1.8)$ \\
\hline $6^{\prime}$ & $6.81 \mathrm{~d}\left(12.0^{e}\right)$ & $6.81 \mathrm{dd}(8.0,1.6)$ & $6.85 \mathrm{~m}$ \\
\hline $1 "$ & $4.84 \mathrm{t}(10.2)$ & $4.81 \mathrm{t}(6.8)$ & $4.95 \mathrm{~d}(7.8)$ \\
\hline $2^{\prime \prime}$ & 3.88 br d $\left(19.2^{e}\right)$ & $3.86 \mathrm{~m}$ & $3.71 \mathrm{~m}$ \\
\hline $3 "$ & $3.80 \mathrm{~m}$ & $3.52 \mathrm{~m}$ & $3.56 \mathrm{~m}$ \\
\hline $4 "$ & 4.99 br s & 3.71 br s & $3.50 \mathrm{~m}$ \\
\hline $5^{\prime \prime}{ }_{a x}$ & 3.68 overlapped & $3.46 \mathrm{~d}(11.2)$ & $3.42 \mathrm{~m}$ \\
\hline $5{ }^{\prime \prime} \mathrm{eq}$ & 3.96 overlapped & $3.79 \mathrm{~m}$ & \\
\hline $6^{\prime \prime}$ & & & $\begin{array}{l}3.85 \mathrm{~m} \\
3.97 \mathrm{brd}(102)\end{array}$ \\
\hline $\mathrm{MeO}-4$ & 3.68 overlapped & $3.67 \mathrm{~s}$ & $3.74 \mathrm{~s}$ \\
\hline $\mathrm{MeO}-5$ & 3.96 overlapped & $3.95 \mathrm{~s}$ & $4.01 \mathrm{~s}$ \\
\hline \multirow[t]{2}{*}{$\mathrm{OCH}_{2} \mathrm{O}-3^{\prime}, 4^{\prime}$} & $6.14 \mathrm{~s}$ & $6.13 \mathrm{br} \mathrm{s}$ & $6.09 \mathrm{~s}$ \\
\hline & $6.14 \mathrm{~s}$ & & $6.10 \mathrm{~s}$ \\
\hline AcO-4" & $2.12 \mathrm{~s}$ & & \\
\hline
\end{tabular}

${ }^{a}$ Chemical shifts were assigned based on the analysis of 1D- and 2D-NMR spectra. The overlapped signals were determined by analysis of the ${ }^{1} \mathrm{H}-{ }^{1} \mathrm{H}$ COSY, HSQC, and HMBC spectra without designating multiplicity ( $\mathrm{s}=$ singlet, $\mathrm{br} \mathrm{s}=$ broad singlet, $\mathrm{d}=$ doublet, $\mathrm{br} \mathrm{d}=$ broad doublet, $\mathrm{dd}=$ double doublet, $\mathrm{t}=$ triplet, $\mathrm{m}=$ multiplet). Proton coupling constant $J$ (in parentheses) values are presented in $\mathrm{Hz}$ and were omitted if the signals overlapped as multiplets.

${ }^{b}$ Data $(\delta)$ recorded at $600.2 \mathrm{MHz}$ in DMSO- $d_{6}$ and referenced to the solvent residual peak at $\delta 2.50{ }^{34}$ ${ }^{c}$ Data $(\delta)$ recorded at $400.1 \mathrm{MHz}$ in DMSO- $d_{6}$ and referenced to the solvent residual peak at $\delta 2.50 .^{34}$

${ }^{d}$ Data $(\delta)$ measured at $600.2 \mathrm{MHz}$ in acetone- $d_{6}$ and referenced to the solvent residual peak at $\delta 2.05 .{ }^{34}$

${ }^{e}$ The unusual value may result from the rotation conformation of the $\mathrm{D}$ ring. 
Table S2. ${ }^{13} \mathrm{C}$ NMR Spectroscopic Data of Compounds of 3-5 ${ }^{a}$

\begin{tabular}{|c|c|c|c|}
\hline position & $3^{b}$ & $4^{c}$ & $5^{d}$ \\
\hline 1 & $126.6 \mathrm{C}$ & $126.6 \mathrm{C}$ & $128.2 \mathrm{C}$ \\
\hline 2 & $129.8 \mathrm{C}$ & $129.7 \mathrm{C}$ & $131.3 \mathrm{C}$ \\
\hline 3 & $105.5 \mathrm{CH}$ & $105.4 \mathrm{CH}$ & $106.6 \mathrm{CH}$ \\
\hline 4 & $150.0 \mathrm{C}$ & $150.0 \mathrm{C}$ & $151.4 \mathrm{C}$ \\
\hline 5 & $151.5 \mathrm{C}$ & $151.4 \mathrm{C}$ & $153.0 \mathrm{C}$ \\
\hline 6 & $101.7 \mathrm{CH}$ & $101.9 \mathrm{CH}$ & $102.6 \mathrm{CH}$ \\
\hline 7 & $144.7 \mathrm{C}$ & $144.6 \mathrm{C}$ & $146.15 / 146.14^{f} \mathrm{C}$ \\
\hline 8 & $129.4 \mathrm{C}$ & $128.98 / 128.81^{f} \mathrm{C}$ & $131.59 / 131.56^{f} \mathrm{C}$ \\
\hline 9 & $67.0 \mathrm{CH}_{2}$ & $67.1 \mathrm{CH}_{2}$ & $68.1 \mathrm{CH}_{2}$ \\
\hline $1^{\prime}$ & $128.2 \mathrm{C}$ & $128.3 \mathrm{C}$ & $129.8 \mathrm{C}$ \\
\hline $2^{\prime}$ & $110.86 / 110.76^{f} \mathrm{CH}$ & $110.91 / 110.83^{f} \mathrm{CH}$ & $111.76 / 111.69^{f} \mathrm{CH}$ \\
\hline $3^{\prime}$ & $146.9 \mathrm{C}$ & $146.9 \mathrm{C}$ & $148.3 \mathrm{C}$ \\
\hline $4^{\prime}$ & $146.9 \mathrm{C}$ & $146.9 \mathrm{C}$ & $148.2 \mathrm{C}$ \\
\hline $5^{\prime}$ & $108.0 \mathrm{CH}$ & $108.0 \mathrm{CH}$ & $108.6 \mathrm{CH}$ \\
\hline $6^{\prime}$ & $123.59 / 123.55^{f} \mathrm{CH}$ & $123.6 \mathrm{CH}$ & $124.5 \mathrm{CH}$ \\
\hline $7^{\prime}$ & $134.9 \mathrm{C}$ & $134.55 / 134.50^{f} \mathrm{C}$ & $136.4 \mathrm{C}$ \\
\hline $8^{\prime}$ & $118.7 \mathrm{C}$ & $118.7 \mathrm{C}$ & $120.1 \mathrm{C}$ \\
\hline $9^{\prime}$ & $169.0 \mathrm{C}$ & $169.1 \mathrm{C}$ & $169.9 \mathrm{C}$ \\
\hline $1^{\prime \prime}$ & $105.14 / 105.06^{f} \mathrm{CH}$ & $104.90 / 104.78^{f} \mathrm{CH}$ & $106.3 \mathrm{CH}$ \\
\hline $2^{\prime \prime}$ & $71.2 \mathrm{CH}$ & $70.8 \mathrm{CH}$ & $75.2 \mathrm{CH}$ \\
\hline 3" & $70.4 \mathrm{CH}$ & $72.3 \mathrm{CH}$ & $78.1 \mathrm{CH}$ \\
\hline $4 "$ & $70.7 \mathrm{CH}$ & $67.32 / 67.27^{f} \mathrm{CH}$ & $71.4 \mathrm{CH}$ \\
\hline $5^{\prime \prime}$ & $63.7 \mathrm{CH}_{2}$ & $65.79 / 65.73^{f} \mathrm{CH}_{2}$ & $78.2 \mathrm{CH}$ \\
\hline $6^{\prime \prime}$ & & & $62.8 \mathrm{CH}_{2}$ \\
\hline MeO-4 & $55.2 \mathrm{CH}_{3}$ & $55.2 \mathrm{CH}_{3}$ & $55.7 \mathrm{CH}_{3}$ \\
\hline $\mathrm{MeO}-5$ & $55.8 \mathrm{CH}_{3}$ & $55.9 \mathrm{CH}_{3}$ & $56.4 \mathrm{CH}_{3}$ \\
\hline $\mathrm{OCH}_{2} \mathrm{O}-3^{\prime}, 4^{\prime}$ & $101.1 \mathrm{CH}_{2}$ & $101.1 \mathrm{CH}_{2}$ & $102.1 \mathrm{CH}_{2}$ \\
\hline \multirow[t]{2}{*}{$\mathrm{AcO}-4 "$} & $170.1 \mathrm{C}^{2}$ & & \\
\hline & $21.1 \mathrm{CH}_{3}$ & & \\
\hline
\end{tabular}

${ }^{a}$ Chemical shifts were assigned based on the analysis of 1D- and 2D-NMR spectra. $\mathrm{CH}_{3}$, $\mathrm{CH}_{2}, \mathrm{CH}$, and $\mathrm{C}$ multiplicities were determined by DEPT 90, DEPT 135, and HSQC experiments.

${ }^{b}$ Data $(\delta)$ measured at $150.9 \mathrm{MHz}$ in DMSO- $d_{6}$ and referenced to the solvent residual peak at $\delta 39.52 .^{34}$

${ }^{c}$ Data $(\delta)$ measured at $100.6 \mathrm{MHz}$ in DMSO- $d_{6}$ and referenced to the solvent residual peak at $\delta 39.52 .^{34}$

${ }^{d}$ Data $(\delta)$ measured at $100.6 \mathrm{MHz}$ in acetone- $d_{6}$ and referenced to the solvent residual peak at $\delta 29.84 .^{34}$

${ }^{f}$ Exist in pairs. 


\section{Table S3. ${ }^{1}$ H NMR Spectroscopic Data of Compounds 6-8 ${ }^{a}$}

\begin{tabular}{|c|c|c|c|}
\hline position & $6^{b}$ & $7^{b}$ & $8^{c}$ \\
\hline 3 & $7.21 \mathrm{~s}$ & $7.07 \mathrm{~s}$ & $7.09 \mathrm{~s}$ \\
\hline 6 & $7.56 \mathrm{~s}$ & $7.54 \mathrm{~s}$ & $7.70 \mathrm{~s}$ \\
\hline 9 & 5.55 br s & $\begin{array}{l}5.50 \mathrm{~d}(15.6) \\
5.44 \mathrm{dd}(14.8,1.6)\end{array}$ & $5.37 \mathrm{~s}$ \\
\hline $2^{\prime}$ & 6.78 overlapped & 6.82 overlapped & $6.85 \mathrm{~d}(1.2)$ \\
\hline $5^{\prime}$ & $6.95 \mathrm{~d}(7.6)$ & $6.97 \mathrm{~d}(8.0)$ & $6.97 \mathrm{~d}(8.0)$ \\
\hline $6^{\prime}$ & 6.78 overlapped & 6.82 overlapped & $6.82 \mathrm{dd}(8.0,1.6)$ \\
\hline $1 "$ & & $5.10 \mathrm{~d}(7.2)$ & \\
\hline $2^{\prime \prime}$ & & $5.72 \mathrm{dd}(9.6,7.2)$ & \\
\hline $3 "$ & & $5.19 \mathrm{dd}(9.6,3.6)$ & \\
\hline 4" & & $5.38 \mathrm{br} \mathrm{s}$ & \\
\hline $5^{\prime \prime}{ }_{a x}$ & & $3.73 \mathrm{brd}(12.8)$ & \\
\hline $5^{\prime \prime}$ & & $4.21 \mathrm{dd}(13.2,2.8)$ & \\
\hline $\mathrm{MeO}-4$ & & $3.81 \mathrm{~s}$ & $3.73 \mathrm{~s}$ \\
\hline $\mathrm{MeO}-5$ & $4.10 \mathrm{~s}$ & $4.09 \mathrm{~s}$ & $4.00 \mathrm{~s}$ \\
\hline $\mathrm{MeO}-7$ & $4.13 \mathrm{~s}$ & & \\
\hline \multirow[t]{2}{*}{$\mathrm{OCH}_{2} \mathrm{O}-3^{\prime}, 4^{\prime}$} & $6.05 \mathrm{~s}$ & $6.05 \mathrm{~s}$ & $6.08 \mathrm{~s}$ \\
\hline & $6.06 \mathrm{~s}$ & $6.10 \mathrm{~s}$ & $6.09 \mathrm{~s}$ \\
\hline AcO-2" & & ${ }^{d} 2.08 \mathrm{~s}$ & \\
\hline AcO-3" & & ${ }^{d} 2.12 \mathrm{~s}$ & \\
\hline AcO-4" & & ${ }^{d} 2.22 \mathrm{~s}$ & \\
\hline $\mathrm{HO}-4$ & $5.96 \mathrm{~s}$ & & \\
\hline
\end{tabular}

${ }^{a}$ Chemical shifts were assigned based on the analysis of 1D- and 2D-NMR spectra. The overlapped signals were determined by analysis of the ${ }^{1} \mathrm{H}-{ }^{1} \mathrm{H}$ COSY, HSQC, and HMBC spectra without designating multiplicity $(\mathrm{s}=$ singlet, $\mathrm{br} \mathrm{s}=$ broad singlet, $\mathrm{d}=\operatorname{doublet}, \mathrm{br} \mathrm{d}=$ broad doublet, $\mathrm{dd}=$ double doublet, $\mathrm{m}=$ multiplet). Proton coupling constant $J$ (in parentheses) values are presented in $\mathrm{Hz}$ and were omitted if the signals overlapped as multiplets.

${ }^{b}$ Data $(\delta)$ recorded at $400.1 \mathrm{MHz}$ in $\mathrm{CDCl}_{3}$ and referenced to the solvent residual peak at $\delta$ $7.26 .^{34}$

${ }^{c}$ Data $(\delta)$ measured at $400.1 \mathrm{MHz}$ in acetone- $d_{6}$ and referenced to the solvent residual peak at $\delta 2.05 .^{34}$

${ }^{d}$ Interchangeable signals. 
Table S4. ${ }^{13} \mathrm{C}$ NMR Spectroscopic Data of Compounds of 6-8 ${ }^{a}$

\begin{tabular}{|c|c|c|c|}
\hline position & $6^{b}$ & $7^{b}$ & $8^{c}$ \\
\hline 1 & $125.8 \mathrm{C}$ & $126.3 \mathrm{C}$ & $124.5 \mathrm{C}$ \\
\hline 2 & $131.4 \mathrm{C}$ & $130.9 \mathrm{C}$ & $131.1 \mathrm{C}$ \\
\hline 3 & $109.9 \mathrm{CH}$ & $106.4 \mathrm{CH}$ & $106.8 \mathrm{CH}$ \\
\hline 4 & $146.8 \mathrm{C}$ & $150.5 \mathrm{C}$ & $151.3 \mathrm{C}$ \\
\hline 5 & $149.4 \mathrm{C}$ & $152.1 \mathrm{C}$ & $152.1 \mathrm{C}$ \\
\hline 6 & $100.3 \mathrm{CH}$ & $100.6 \mathrm{CH}$ & $101.3 \mathrm{CH}$ \\
\hline 7 & $148.0 \mathrm{C}$ & $144.3 \mathrm{C}$ & $145.7 \mathrm{C}$ \\
\hline 8 & $123.9 \mathrm{C}$ & $127.4 \mathrm{C}$ & $122.8 \mathrm{C}$ \\
\hline 9 & $66.8 \mathrm{CH}_{2}$ & $67.0 \mathrm{CH}_{2}$ & $67.0 \mathrm{CH}_{2}$ \\
\hline $1^{\prime}$ & $128.5 \mathrm{C}$ & $128.3 \mathrm{C}$ & $130.2 \mathrm{C}$ \\
\hline $2^{\prime}$ & $111.0 \mathrm{CH}$ & $110.8 \mathrm{CH}$ & $112.0 \mathrm{CH}$ \\
\hline $3^{\prime}$ & $147.5 \mathrm{C}$ & $147.7 \mathrm{C}$ & $148.3 \mathrm{C}$ \\
\hline $4^{\prime}$ & $147.5 \mathrm{C}$ & $147.7 \mathrm{C}$ & $148.0 \mathrm{C}$ \\
\hline $5^{\prime}$ & $108.3 \mathrm{CH}$ & $108.4 \mathrm{CH}$ & $108.6 \mathrm{CH}$ \\
\hline $6^{\prime}$ & $123.8 \mathrm{CH}$ & $123.69 / 123.67 \mathrm{CH}^{e}$ & $124.8 \mathrm{CH}$ \\
\hline $7^{\prime}$ & $134.8 \mathrm{C}$ & $136.4 \mathrm{C}$ & $131.6 \mathrm{C}$ \\
\hline $8^{\prime}$ & $119.6 \mathrm{C}$ & $119.4 \mathrm{C}$ & $120.0 \mathrm{C}$ \\
\hline $9^{\prime}$ & $169.7 \mathrm{C}$ & $169.6 \mathrm{C}$ & $170.2 \mathrm{C}$ \\
\hline $1^{\prime \prime}$ & & $101.6 \mathrm{CH}$ & \\
\hline $2^{\prime \prime}$ & & $69.5 \mathrm{CH}$ & \\
\hline $3^{\prime \prime}$ & & $70.3 \mathrm{CH}$ & \\
\hline $4 "$ & & $67.39 / 67.01 \mathrm{CH}^{e}$ & \\
\hline $5 "$ & & $64.1 \mathrm{CH}_{2}$ & \\
\hline $\mathrm{MeO}-4$ & & $56.0 \mathrm{CH}_{3}$ & $55.7 \mathrm{CH}_{3}$ \\
\hline MeO-5 & $56.4 \mathrm{CH}_{3}$ & $56.4 \mathrm{CH}_{3}$ & $56.1 \mathrm{CH}_{3}$ \\
\hline $\mathrm{MeO}-7$ & $59.8 \mathrm{CH}_{3}$ & & \\
\hline $\mathrm{OCH}_{2} \mathrm{O}-3^{\prime}, 4^{\prime}$ & $101.3 \mathrm{CH}_{2}$ & $101.4 \mathrm{CH}_{2}$ & $102.1 \mathrm{CH}_{2}$ \\
\hline \multirow{2}{*}{$\mathrm{AcO}-2^{\prime \prime}$} & & ${ }^{f} 170.4 \mathrm{C}$ & \\
\hline & & ${ }^{g} 20.9 \mathrm{CH} 3$ & \\
\hline \multirow[t]{2}{*}{ AcO-3" } & & ${ }^{f} 170.3 \mathrm{C}$ & \\
\hline & & ${ }^{g} 21.1 \mathrm{CH}_{3}$ & \\
\hline \multirow[t]{2}{*}{ AcO-4" } & & ${ }^{f} 169.6 \mathrm{C}$ & \\
\hline & & ${ }^{g} 21.1 \mathrm{CH}_{3}$ & \\
\hline
\end{tabular}

${ }^{a}$ Chemical shifts were assigned based on the analysis of 1D- and 2D-NMR spectra. $\mathrm{CH}_{3}, \mathrm{CH}_{2}, \mathrm{CH}$, and $\mathrm{C}$ multiplicities were determined by DEPT 90, DEPT 135, and HSQC experiments.

${ }^{b}$ Data $(\delta)$ measured at $100.6 \mathrm{MHz}$ in $\mathrm{CDCl}_{3}$ and referenced to the solvent residual peak at $\delta 77.16 .^{34}$

${ }^{c}$ Data $(\delta)$ measured at $100.6 \mathrm{MHz}$ in acetone- $d_{6}$ and referenced to the solvent residual peak at $\delta 29.84 .^{34}$

${ }^{e}$ Exist in pairs.

${ }^{f}$ Interchangeable signals.

${ }^{g}$ Interchangeable signals. 
Analytical data of known compounds (3-8) isolated from Phyllanthus poilanei.

Phyllanthusmin B (3): Amorphous colorless powder showing a blue color under UV light at 365 $\mathrm{nm} ;[\alpha]^{20}{ }_{\mathrm{D}}-6\left(c 0.1, \mathrm{CHCl}_{3}\right) ; \mathrm{UV}(\mathrm{MeOH}) \lambda_{\max }(\log \varepsilon) 260(4.62) \mathrm{nm} ; \mathrm{ECD}(\mathrm{MeOH}, \mathrm{nm}) \lambda_{\max }$ ( $\Delta \varepsilon) 297$ (-4.12); IR (dried film) $v_{\max } 3364,1723,1615,1505,1480,765 \mathrm{~cm}^{-1} ;{ }^{1} \mathrm{H}$ and ${ }^{13} \mathrm{C}$ NMR data, see Tables S1 and S2; positive-ion HRESIMS $m / z$ 577.1317, calcd for $\mathrm{C}_{28} \mathrm{H}_{26} \mathrm{O}_{12} \mathrm{Na}$, 577.1316.

Phyllanthusmin C (4): Amorphous colorless powder showing a blue color under UV light at 365 $\mathrm{nm} ;[\alpha]^{20}{ }_{\mathrm{D}}-8\left(c 0.1, \mathrm{CHCl}_{3}\right) ; \mathrm{UV}(\mathrm{MeOH}) \lambda_{\max }(\log \varepsilon) 260(4.35) \mathrm{nm} ; \mathrm{ECD}(\mathrm{MeOH}, \mathrm{nm}) \lambda_{\max }$ ( $\Delta \varepsilon) 292$ (-3.19); IR (dried film) $v_{\max } 3373,1734,1619,1507,1480,767 \mathrm{~cm}^{-1} ;{ }^{1} \mathrm{H}$ and ${ }^{13} \mathrm{C}$ NMR data, see Tables S1 and S2; positive-ion HRESIMS $m / z$ 535.1237, calcd for $\mathrm{C}_{26} \mathrm{H}_{24} \mathrm{O}_{11} \mathrm{Na}$, 535.1210.

Cleistanthin B (5): Amorphous colorless powder showing a blue color under UV light at $365 \mathrm{~nm}$; $[\alpha]^{20}{ }_{D}-53(c 0.1, \mathrm{MeOH}) ; \mathrm{UV}(\mathrm{MeOH}) \lambda_{\max }(\log \varepsilon) 260(4.73) \mathrm{nm} ; \mathrm{ECD}(\mathrm{MeOH}, \mathrm{nm}) \lambda_{\max }(\Delta \varepsilon)$ 301 (-4.94); IR (dried film) $v_{\max } 3390,1739,1713,1622,1506,1481,770 \mathrm{~cm}^{-1} ;{ }^{1} \mathrm{H}$ and ${ }^{13} \mathrm{C}$ NMR data, see Tables $\mathrm{S} 1$ and S2; positive-ion HRESIMS $m / z$ 565.1321, calcd for $\mathrm{C}_{27} \mathrm{H}_{26} \mathrm{O}_{12} \mathrm{Na}$, 565.1316.

Phyllanthusmin A (6): Amorphous colorless powder showing a blue color under UV light at 365 nm; UV (MeOH) $\lambda_{\max }(\log \varepsilon) 262(4.61) \mathrm{nm}$; IR (dried film) $v_{\max }$ 3447, 1766, 1716, 1597, 1508, 1480, $752 \mathrm{~cm}^{-1} ;{ }^{1} \mathrm{H}$ and ${ }^{13} \mathrm{C}$ NMR data, see Tables S3 and S4; positive-ion HRESIMS $\mathrm{m} / \mathrm{z}$ 403.0794, calcd for $\mathrm{C}_{21} \mathrm{H}_{16} \mathrm{O}_{7} \mathrm{Na}, 403.0788$.

7-O-[(2,3,4-tri-O-acetyl)- $\alpha$-L-arabinopyranosyl)]diphyllin (7): Amorphous colorless powder showing a blue color under UV light at $365 \mathrm{~nm} ;[\alpha]^{20}{ }_{\mathrm{D}}-12\left(c 0.1, \mathrm{CHCl}_{3}\right) ; \mathrm{UV}(\mathrm{MeOH}) \lambda \max$ $(\log \varepsilon) 260(4.45) \mathrm{nm} ; \mathrm{ECD}(\mathrm{MeOH}, \mathrm{nm}) \lambda \max (\Delta \varepsilon) 295$ (-3.87); IR (dried film) $v_{\max }$ 1749, 1619, 
1506, 1480, $770 \mathrm{~cm}-1 ;{ }^{1} \mathrm{H}$ and ${ }^{13} \mathrm{C}$ NMR data, see Tables S3 and S4; positive-ion HRESIMS m/z 661.1553, calcd for $\mathrm{C}_{32} \mathrm{H}_{30} \mathrm{O}_{14} \mathrm{Na}, 661.1527$.

Diphyllin (8): Amorphous colorless powder showing a blue color under UV light at $365 \mathrm{~nm}$; UV $(\mathrm{MeOH}) \lambda_{\max }(\log \varepsilon) 267(4.59) \mathrm{nm}$; IR (dried film) $v_{\max } 1705,1615,1506,1489,774 \mathrm{~cm}^{-1} ;{ }^{1} \mathrm{H}$ and ${ }^{13} \mathrm{C}$ NMR data, see Tables S3 and S4; positive-ion HRESIMS $m / z$ 403.0797, calcd for $\mathrm{C}_{21} \mathrm{H}_{16} \mathrm{O}_{7} \mathrm{Na}, 403.0788$. 